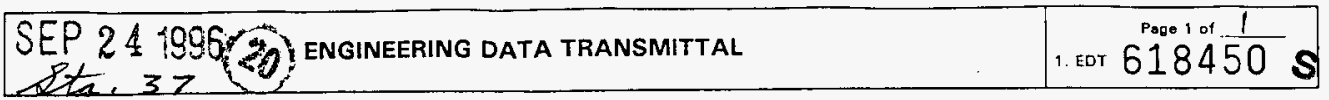

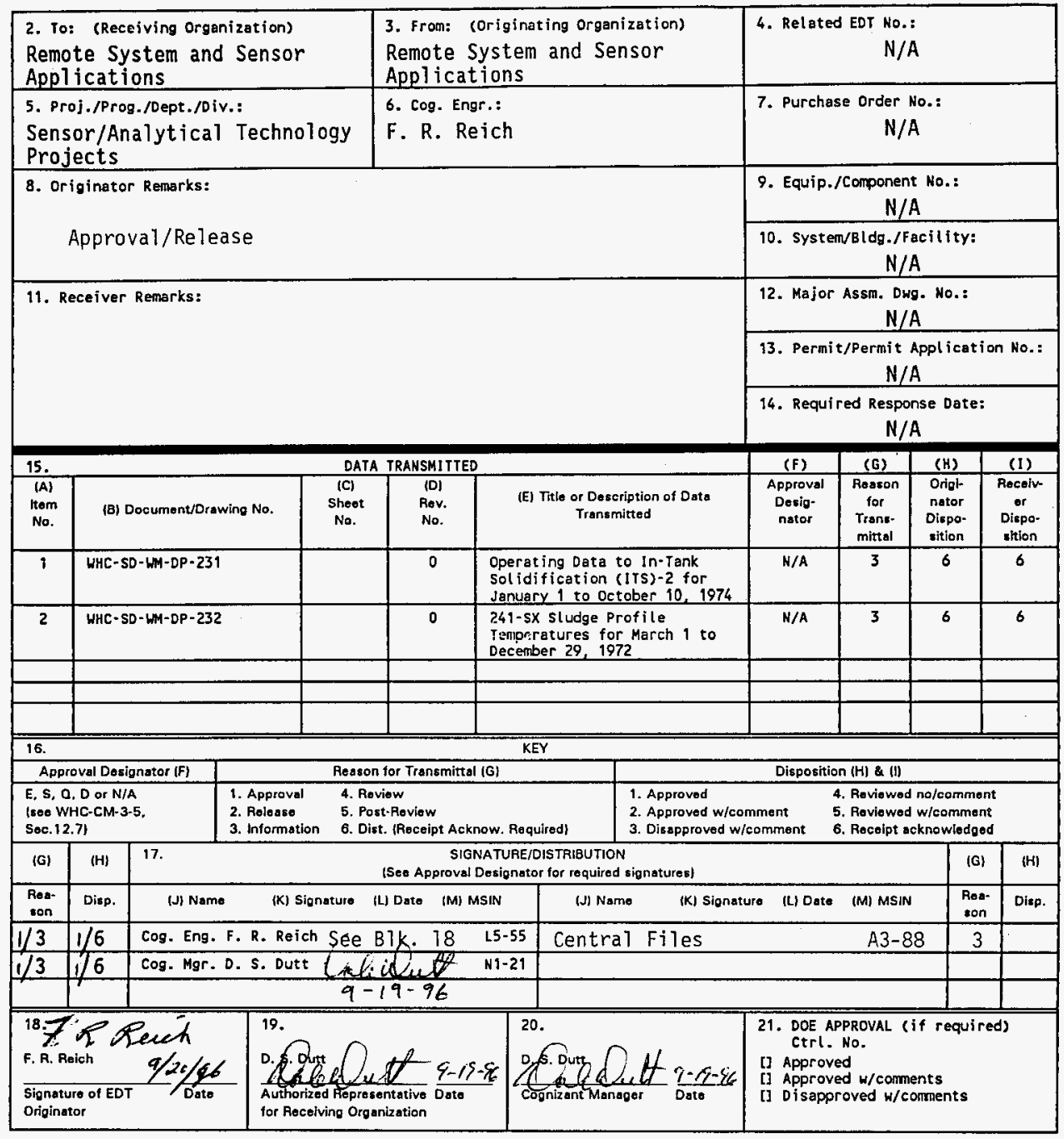

BD-7400-172-2 (04/94) GEF097 


\title{
241-SX Sludge Profile Temperatures for March 1 to December 29, 1972
}

\author{
F. R. Reich
}

Westinghouse Hanford Company, Richland, WA 99352

U.S. Department of Energy Contract DE-AC06-87RL10930

$\begin{array}{lll}\text { EDT/ECN: } & 618450 & \text { UC: } 721 \\ \text { Org Code: } & 8 A 800 & \text { Charge Code: } \\ \text { B\&R Code: } & \text { EW4010000 } & \text { Total Pages: } 135\end{array}$

Key Words: 241-SX, Sludge Profile Temperatures

Abstract: Data sheets containing 24l-SX sludge profile temperatures.

TRADEMARK DISCLAIMER. Reference herein to any specific commercial product, process, or service by trade name, trademark, manufacturer, or otherwise, does not necessarily constitute or imply its endorsement, recommendation, or favoring by the United States Government or any agency thereof or its contractors or subcontractors.

Printed in the United States of America. To obtain copies of this document, contact: HHC/BCS

Document Control Services, P.O. Box 1970, Mailstop H6-08, Richland wA 99352, Phone (509) 372-2420; Fax (509) 376-4989.
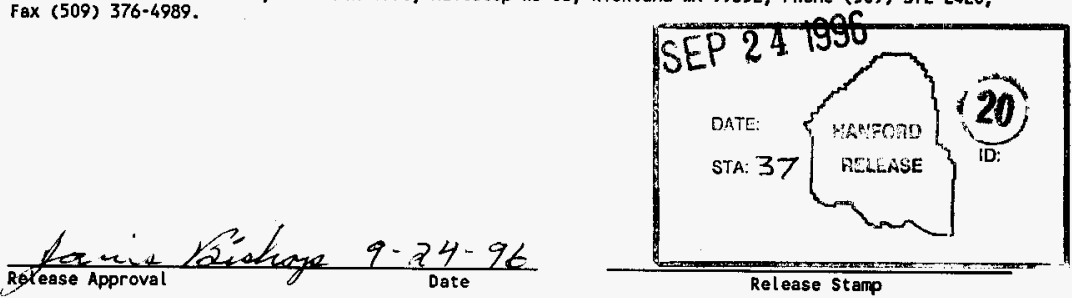


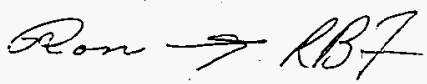

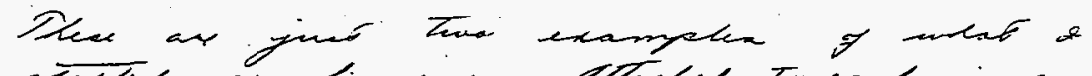

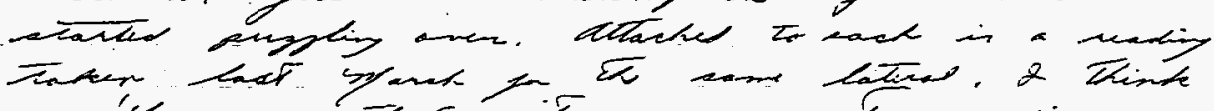

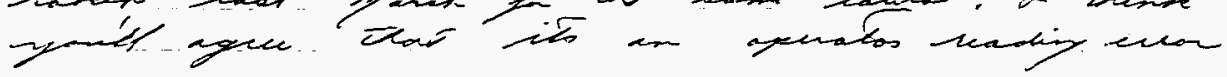

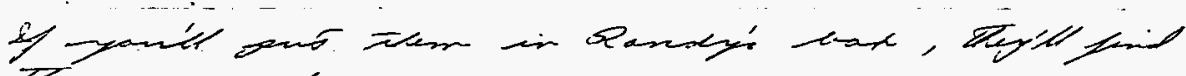
Lis ary tome.

S

$0270 \rightarrow-0 \times 30 \ldots \ldots+1-10 \leqslant A \ldots$

$.093 x \rightarrow 102 x$

3.14159
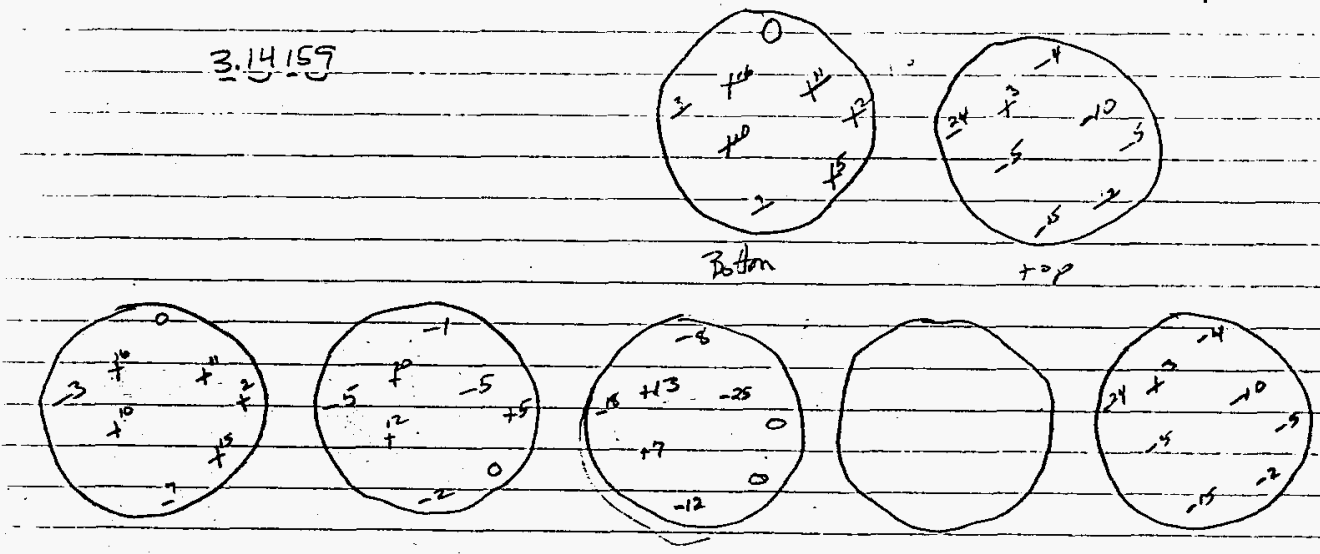

2

3

4 
WHC-SO-LM-DP-232, ReV. $O$

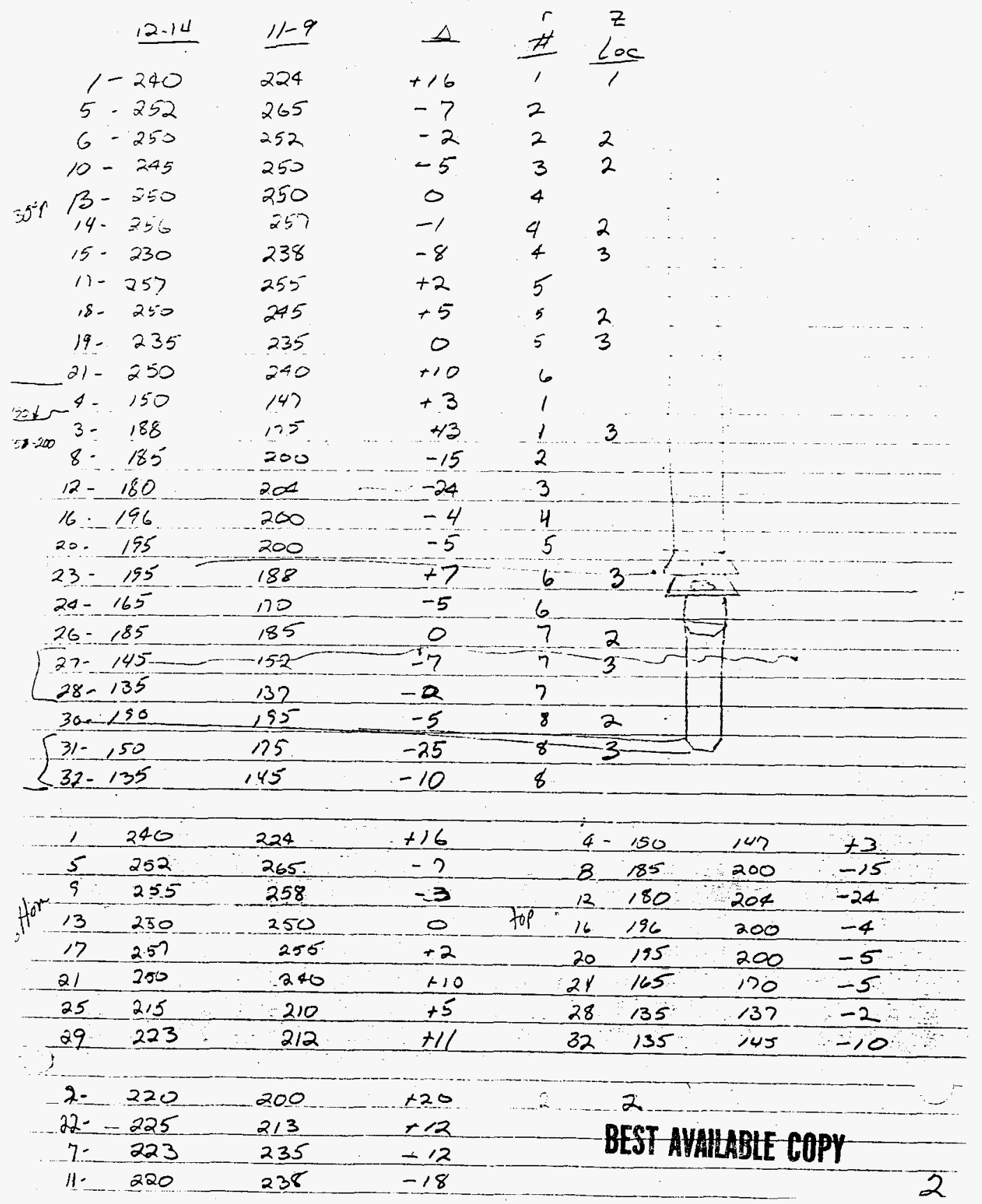


241-SX SLUDGE PROEILE TEMPERATURES

(S.0.P. 200.3.6)

Time

Date

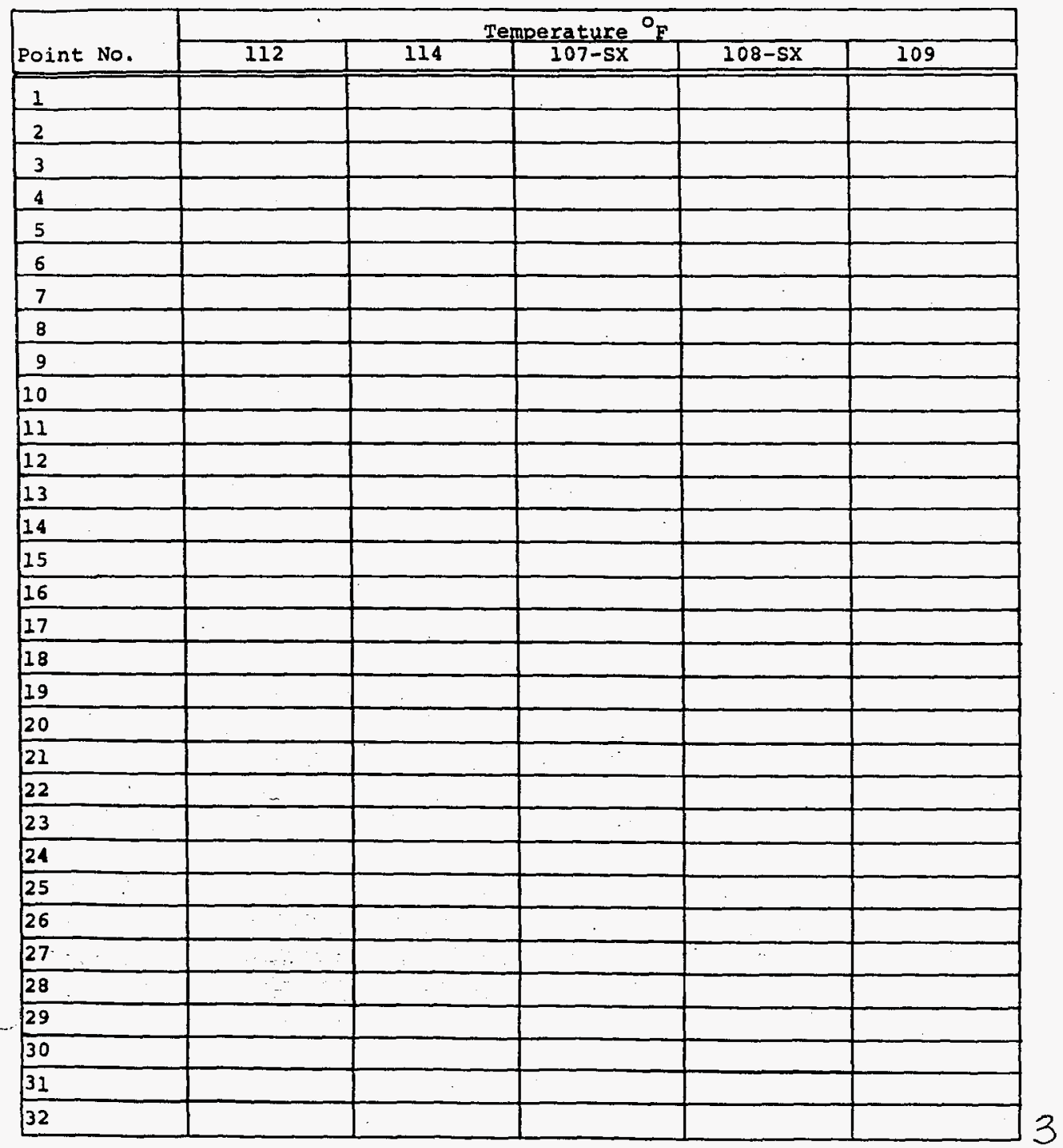


241-SX SLUDGE PROFILE TEMPERATURES

(S.0.P. 200.3.6)

Time $\frac{2 / 15}{19 / 29 / 32}$

\begin{tabular}{|c|c|c|c|c|c|}
\hline \multirow[b]{2}{*}{ Point No. } & \multicolumn{5}{|c|}{ Temperature ${ }^{\circ} \mathrm{F}$} \\
\hline & 112 & 114 & $107-5 x$ & $108-5 x$ & 109 \\
\hline 1 & $\angle 51$ & 240 & 142 & 135 & $1<5$ \\
\hline 2 & 137 & 211 & 100 & 96 & $\angle<5$ \\
\hline 3 & $110^{\circ}$ & 175 & 24 & $\$ 0$ & 155 \\
\hline 4 & 80 & $\angle 50$ & 51 & 28 & $\angle 35$ \\
\hline$\underline{5}$ & 115 & 260 & 151 & $\angle 5 C$ & 137 \\
\hline 6 & $\angle O Z$ & 247 & 124 & $\angle 30$ & $\angle 32$ \\
\hline 7 & 93 & 222 & $\Sigma \%$ & 85 & 112 \\
\hline 8 & 80 & $\angle 85$ & 56 & 22 & 99 \\
\hline 9 & 100 & 252 & 168 & 65 & $<<9$ \\
\hline 10 & 85 & 243 & 140 & $29(27)$ & 122 \\
\hline 11 & 33 & 217 & SZ & 80 & $\angle<8$ \\
\hline 12 & 65 & 180 & 50 & $2<$ & $\angle 50$ \\
\hline 13 & 105 & 248 & 165 & $\angle 31$ & 149 \\
\hline 14 & 92 & 255 & 32 & 81 & 147 \\
\hline 15 & 75 & 222 & $2 C$ & 25 & 133 \\
\hline 16 & 63 & $\angle E 7$ & 50 & 25 & 119 \\
\hline 17 & 27 & 255 & 154 & $\angle 45$ & $\angle 37$ \\
\hline 18 & 85 & 243 & 130 & & $13<$ \\
\hline 19 & 23 & 229 & 25 & 81 & $\angle 33$ \\
\hline 20 & 65 & 192 & 53 & 22 & 127 \\
\hline 21 & $<42$ & 244 & $\angle 40$ & $N 6$ & $\angle 36$ \\
\hline 22 & $\angle 30$ & 218 & 114 & $\longleftarrow$ & 127 \\
\hline 23 & 185 & $\angle 84$ & 90 & $\angle$ & 113 \\
\hline 24 & 90 & 155 & 65 & 82 & 89 \\
\hline 25 & $\angle 05$ & 212 & 27 & 143 & $\angle 40$ \\
\hline 26 & 88 & 187 & $\angle 0$ & 118 & 36 \\
\hline 27 & 75 & 149 & 51 & 81 & $\angle 20$ \\
\hline 28 & 67 & 137 & 49 & 22 & 100 \\
\hline 29 & 125 & 224 & $\angle 37$ & $\angle 61$ & $\angle 4 Q$ \\
\hline 30 & $\angle 05$ & $\angle 88$ & $1 \pm 4$ & $\angle 17$ & $\angle 20$ \\
\hline 31 & 85 & $\angle 39$ & 57 & 75 & 110 \\
\hline 32 & 23 & $\angle 36$ & 50 & 75 & 90 \\
\hline
\end{tabular}


241-SX SLUDGE PROFILE TEMPERATURES

(S.0.P. 200.3.6)

Time $\frac{2 / 20}{12 / 25 / 22}$

\begin{tabular}{|c|c|c|c|c|c|}
\hline \multirow[b]{2}{*}{ Point No. } & \multicolumn{5}{|c|}{ Temperature ${ }^{\circ} \mathrm{F}$} \\
\hline & 112 & 114 & $107-S x$ & $108-S x$ & 109 \\
\hline 1 & $15346^{2}$ & 240 & 145 & 1.35 & $165 \times 69$ \\
\hline 2 & 132 & $2 / 0$ & 100 & 95 & $165 v$ \\
\hline 3 & 110 & 173 & 23 & 80 & 153 \\
\hline 4 & $90<$ & 148 & 52 & 28 & 133 \\
\hline 5 & 115 & 260 & 150 & 156 & 137 \\
\hline 6 & 108 & 247 & 124 & 139 & 131 \\
\hline 7 & 93 & 223 & 83 & 85 & 116 \\
\hline 8 & 80 & 185 & 53 & 77 & 98 \\
\hline 9 & 100 & 252 & $167(13)$ & $16.5 \times 64$ & 163 \\
\hline 10 & 85 & 243 & 138 & 129 & 167 \\
\hline 11 & 73 & 217 & 88 & 82 & 162 \\
\hline 12 & 65 & 180 & 50 & 27 & 150 \\
\hline 13 & 109 & 248 & 165 & 127 & 147 \\
\hline 14 & 93 & 255 & 132 & 82 & 147 \\
\hline 15 & 35 & 227 & 76 & 77 & 137 \\
\hline 16 & 20 & $188^{\prime}$ & 50 & 77 & 118 \\
\hline 17 & 99 & 253 & 154 & $\angle 45^{\prime}$ & 137 \\
\hline 18 & 85 & 248 & 127 & 121 & 137 \\
\hline 19 & 75 & 230 & 75 & 82 & 133 \\
\hline 20 & 65 & 192 & 54 & 78 & 127 \\
\hline 21 & 145 & 243 & 140 & $N 6$ & 137 \\
\hline 22 & 130 & 218 & 113 & $N 6$ & 127 \\
\hline 23 & 110 & 183 & 91 & $N 6$ & 113 \\
\hline 24 & 93 & 155 & 67 & $\sqrt{6}$ & $98^{8}$ \\
\hline 25 & 107 & 212 & 77 & 143 & 140 \\
\hline 26 & 90 & 183 & 60 & 117 & 136 \\
\hline 27 & 75 & 149 & 52 & 80 & 120 \\
\hline $28-$ & 67 & 135 & 50 & 72 & 100 \\
\hline 29 & 125 & 223 & 137 & 175 & 140 \\
\hline 30 & 107 & 190 & 123 & 115 & 127 \\
\hline 31 & $N-6$ & 140 & 58 & 76 & 110 \\
\hline 32 & 75 & 136 & 57 & 76 & 90 \\
\hline
\end{tabular}


241-SX SLUDGE PROFILE TEMPERATURES

(S.0.P. 200.3.6)

Time $\frac{1100}{19 / 22 / 2_{2}}$

\begin{tabular}{|c|c|c|c|c|c|}
\hline \multirow[b]{2}{*}{ Point No. } & \multicolumn{5}{|c|}{ Temperature ${ }^{\circ} \mathrm{F}$} \\
\hline & 112 & 114 & $107-5 x$ & $108-5 X$ & 109 \\
\hline 1 & $(1) / 52$ & 240 & 145 & 34 & 5 \\
\hline 2 & $\angle 30$ & $2 / 2$ & 100 & 99 & 65 \\
\hline 3 & 110 & 123 & 25 & 10 & 54 \\
\hline 4 & 90 & 146 & & 7 & 35 \\
\hline 5 & 115 & 260 & 150 & $3-$ & 37 \\
\hline 6 & 102 & 248 & 125 & & 132 \\
\hline 7 & 93 & 222 & 83 & & 115 \\
\hline 8 & 50 & 185 & 5 & & \\
\hline 9 & 100 & 252 & $170^{\circ}(4)$ & $3^{n}\left(v_{1}\right)$ & 162 \\
\hline 10 & 85 & 244 & $\angle 37$ & & \\
\hline 11 & 20 & 217 & & 2 & 162 \\
\hline 12 & 15 & 180 & 52 & & \\
\hline 13 & $\angle 25$ & 248 & 165 & 20 & 5 \\
\hline 24 & 90 & 255 & 10 & 2 & 2 \\
\hline 15 & 25 & 229 & & & 32 \\
\hline 16 & 68 & 189 & $5-3$ & 26 & 18 \\
\hline 17 & 96 & 253 & 52 & - & 2 \\
\hline 18 & 83 & $2<1$ & & 2 & \\
\hline 19 & 23 & 232 & & 2 & 128 \\
\hline 20 & 66 & 192 & 55 & 21 & $\angle 22$ \\
\hline 21 & 145 & 245 & 140 & 25 & 132 \\
\hline 22 & $\angle 28$ & 218 & 11 & 20 & 2 \\
\hline 23 & $\angle 8 q$ & $\angle 82$ & & & 113 \\
\hline 24 & 90 & 152 & 20 & 85 & \\
\hline 25 & 105 & $2 / 3$ & 25 & & 141 \\
\hline 26 & 88 & 182 & $\angle 2$ & 12 & 139 \\
\hline 27 & 25 & 143 & $s^{-4}$ & 80 & $\angle 20$ \\
\hline 28 & 69 & $\angle 35$ & 53 & 27 & 100 \\
\hline 29 & 125 & 223 & $\angle 33$ & & 140 \\
\hline 30 & 105 & 180 & 123 & & 181 \\
\hline 31 & 85 & 145 & 60 & 25 & 110 \\
\hline $32=$ & 23 & 135 & 5 & 23 & 90 \\
\hline
\end{tabular}


241-SX SLUDGE PROFILE TEMPERATURES

(S.0.P. 200.3.6)

Time $\frac{1040}{12-21-72}$

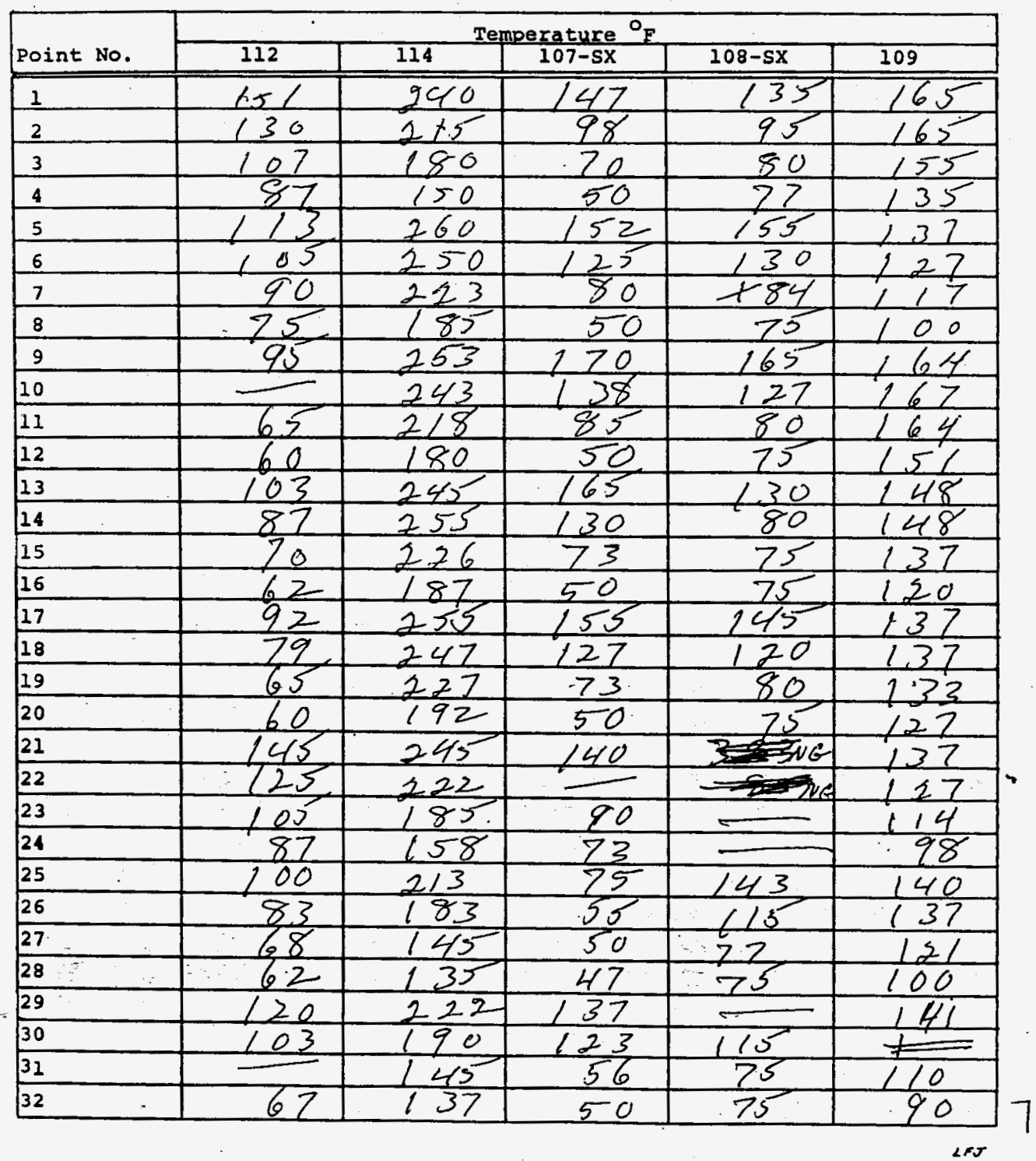


241-SX SLUDGE PROFILE TEMPERATURES

(S.0.P. 200.3.6)

Time 0935

Date $11-14 \cdot 72$

\begin{tabular}{|c|c|c|c|c|c|}
\hline \multirow[b]{2}{*}{ Point No. } & \multicolumn{5}{|c|}{$\frac{\text { Temperature }}{107-5 X}$} \\
\hline & 112 & 114 & $107-5 x$ & $108-5 x$ & 109 \\
\hline 1 & 152 & 240 & 150 & 1.53 & 65 \\
\hline 2 & 130 & 220 & 95 & 90 & 165 \\
\hline 3 & 100 & 188 & 100 & 74 & 155 \\
\hline 4 & 75 & 150 & 35 & 21 & 136 \\
\hline 5 & 115 & 2.52 & 25 & 256 & 139 \\
\hline 6 & 104 & 250 & & 127 & 133 \\
\hline 7 & $=$ & 223 & & 79 & 120 \\
\hline 6 & 18 & 185 & 3 & 70 & 100 \\
\hline 9 & 90 & 255 & 170 & & 165 \\
\hline 12 & 70 & 245 & & 12 & 168 \\
\hline 11 & 55 & 220 & 8 & 74 & 65 \\
\hline 12 & 4,5 & 180 & 2 & 70 & 152 \\
\hline 13 & 100 & 2.50 & 167 & 30 & 148 \\
\hline 14 & 80 & 256 & 130 & 13 & 150 \\
\hline 15 & 60 & 230 & 63 & 70 & 140 \\
\hline 16 & 50 & 196 & 3 & 30 & 121 \\
\hline 17 & 90 & 257 & 15 & 145 & 140 \\
\hline 18 & 72 & 2.50 & 12 & 120 & 1.40 \\
\hline 19 & 57 & 235 & 62 & 78 & 135 \\
\hline 20 & 47 & 1.95 & $\frac{3}{2}$ & $\geq 3$ & 130 \\
\hline 21 & 146 & 250 & 140 & & 140 \\
\hline 22 & 126 & 225 & 115 & & 130 \\
\hline 23 & 100 & 195 & 85 & .90 & \\
\hline 24 & 76 & 165 & 54 & & 100 \\
\hline $25: \quad \therefore 25$ & 100 & 215 & 5367 & 145 & 142 \\
\hline 26 & 76 & 185 & 24 & 115 & 138 \\
\hline 21 & 660 & 145 & 321 & 75 & 125 \\
\hline $28=28$ & 50 & 135 & $3-30$ & 71 & 103 \\
\hline $29 \cdots 29$ & 119 & 223 & 13 & 177 & 143 \\
\hline 30 & 200 & 190 & $123=0$ & 174 & 1,21 \\
\hline 31 & $\because$ & 150 & $41 \Rightarrow$ & 70 & $1 / 5$ \\
\hline 32 & 51 & 135 & 354 & 70 & 94 \\
\hline
\end{tabular}


241-SX SLUDGE PROFILE TEMPERATURES

(S.0.P. 200.3.6)
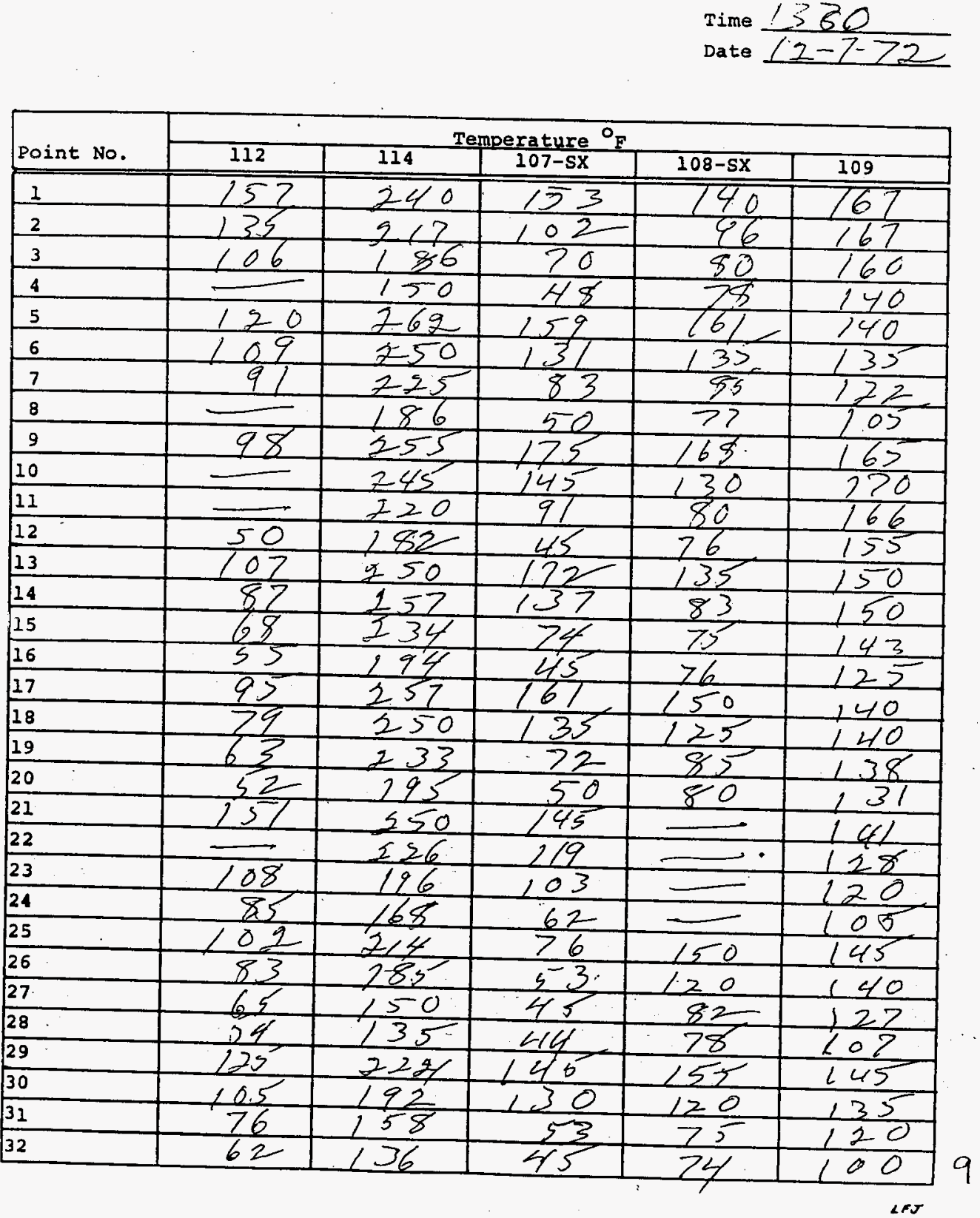
241-SX SLUDGE PROFILE TEMPERATURES

(S.0.P. 200.3.6)

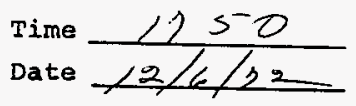

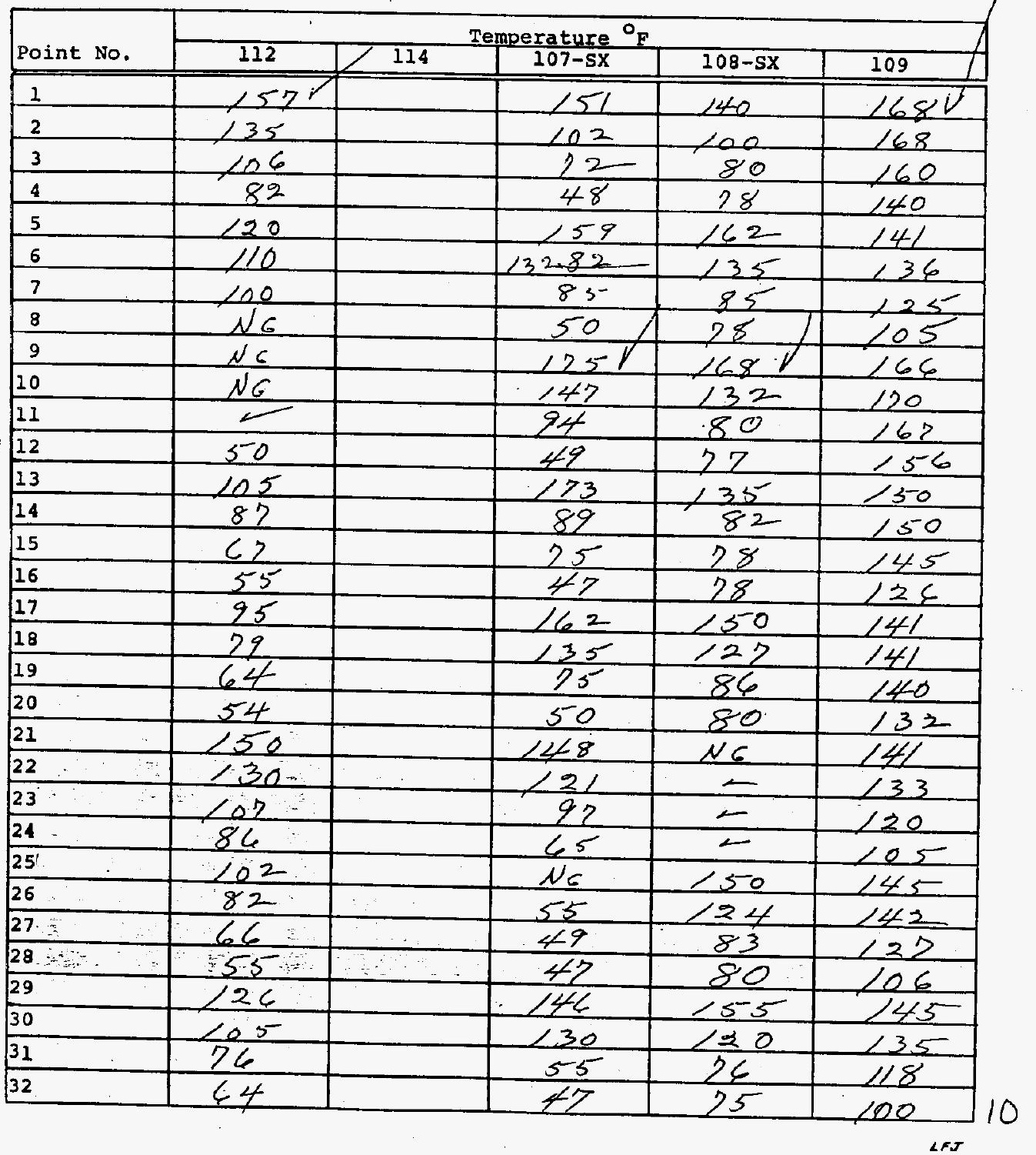


241-SX SLUDGE PROFILE TEMPERATURES

(S.0.P. 200.3.6)

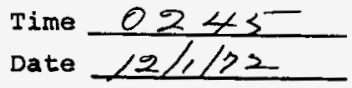

\begin{tabular}{|c|c|c|c|c|c|}
\hline \multirow[b]{2}{*}{ Point No. } & \multicolumn{5}{|c|}{ Temperature ${ }^{\circ} F$} \\
\hline & 112 & 114 & $107-5 x$ & $108-5 x$ & 109 \\
\hline 1 & 160 & 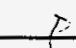 & 254 & 141 & 168 \\
\hline 2 & $\angle 32$ & & $\angle 06$ & 100 & 168 \\
\hline 3 & $\angle 10$ & & 26 & $\$ 5$ & 160 \\
\hline 4 & 86 & 2 & 55 & 83 & 142 \\
\hline 5 & $\angle 20$ & 2 & 160 & $\angle 62$ & 142 \\
\hline 6 & 112 & & 83 & 136 & 137 \\
\hline 7 & 95 & & 90 & 91. & 124 \\
\hline 8 & 79 & & 55 & 82 & 105 \\
\hline 9 & $\angle 00$ & & 175 & 168 & 166 \\
\hline 10 & 82 & & $\angle \angle Q$ & $\angle 33$ & 170 \\
\hline 11 & 65 & & 99 & 86 & 167 \\
\hline 12 & 55 & & +254 & 81 & $\angle 56$ \\
\hline 13 & $\angle 10$ & & 123 & 136 & $\angle 50$ \\
\hline 14 & 90 & & $\angle 40$ & 82 & 150 \\
\hline 15 & 79 & & 80 & 81 & 243 \\
\hline 16 & $\cos$ & & 51 & 81 & $\angle 26$ \\
\hline 27 & 100 & & 162 & 150 & 140 \\
\hline 18 & 80 & & 136 & 127 & 140 \\
\hline 19 & 68 & & 39 & 88 & 138 \\
\hline 20 & 58 & & 53 & 85 & 132 \\
\hline 21 & $\angle 30$ & & $\angle 48$ & $\Delta<$ & 141 \\
\hline 22 & $\angle 35$ & & $\angle 22$ & .1 & $\angle 32$ \\
\hline 23 & $\angle \angle O$ & & 99 & .1 & 120 \\
\hline 24 & 58 & & 70 & 11 & 125 \\
\hline 25 . & 105 & & 27 & 150 & $\angle 43$ \\
\hline 26 & 88 & & 60 & 125 & 1412 \\
\hline $27 \cdots$ & 23 & & 52 & 87 & $\angle 22$ \\
\hline 28 & 100 & & $5 \%$ & 83 & 182 \\
\hline 29 & $\angle 26$ & & 147 & $N \cdot \angle x=G$ & 145 \\
\hline 30 & 107 & & 132 & $\angle 83$ & $\angle 35$ \\
\hline 31 & 85 & $\sum$ & 61 & 80 & 118 \\
\hline 32 & 68 & $\nabla$ & 52 & 80 & 100 \\
\hline
\end{tabular}


241-SX SLUDGE PROEIIE TEMPERATURES

(S.O.P. 200.3.6)

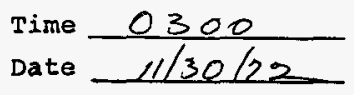

\begin{tabular}{|c|c|c|c|c|c|}
\hline \multirow[b]{2}{*}{ Point No. } & \multicolumn{3}{|c|}{ Temperature ${ }^{{ }^{\circ} F}$} & \multirow[b]{2}{*}{$108-5 x$} & \multirow[b]{2}{*}{109} \\
\hline & 112 & 114 & $107-5 x$ & & \\
\hline 1 & $160 x$ & (f) & 155 & $\angle 42$ & 168 \\
\hline 2 & 135 & $\sigma$ & $\angle 07$ & $\angle 2=$ & 168 \\
\hline 3 & 112 & & 27 & 82 & 160 \\
\hline 4 & 87 & & 55 & 85 & 141 \\
\hline 5 & $\angle 90$ & & $1 \cos$ & 163 & $|4|$ \\
\hline 6 & 114 & & $\angle 35$ & 132 & 138 \\
\hline 7 & 96 & & 20 & 83 & $\angle 25$ \\
\hline 8 & 50 & & 57 & 285 & $<\gamma 7$ \\
\hline 9 & $\angle 00$ & & $126 \times 1$ & $80 / 200^{\circ}$ & $\angle 66 A_{1}$ \\
\hline 10 & 84 & & 150 & $<3 s$ & 130 \\
\hline 11 & 20 & & $\angle 20$ & 52 & 162 \\
\hline 12 & 34 & & 55 & 85 & 157 \\
\hline 13 & 110 & & 224 & $\angle 39$ & 150 \\
\hline 14 & 90 & & 142 & 88 & $\angle 50$ \\
\hline 15 & 20 & & 82 & 83 & 144 \\
\hline 16 & 60 & & 53 & 83 & $1 \geq 6$ \\
\hline 17 & $\angle 00$ & & $\angle 63$ & 150 & 241 \\
\hline 18 & $\$ 0$ & & 132 & $\angle 30$ & 141 \\
\hline 19 & 70 & & 80 & 90 & 139 \\
\hline 20 & 62 & & 56 & 85 & 233 \\
\hline 21 & 250 & & $\angle 48$ & $\Delta 6$ & $\angle 43$ \\
\hline 22 & $\angle 35$ & & 124 & .1 & $\angle 34$ \\
\hline 23 & 110 & & 100 & $\because 1$ & $\angle 21$ \\
\hline 24 & 90 & & 70 & 11 & $<05$ \\
\hline 25 & $\angle 05$ & & 29 & 150 & 145 \\
\hline 26 & 80 & $\because$ & $\therefore 62$ & 125 & 142 \\
\hline $27 \vdots$ & 20 & $\mathrm{i}$ & $\therefore 55$ & $\therefore 88$ & $\angle 28$ \\
\hline 28 & $\cos :$ & 2 & $23^{-4}$ & 54 & 108 \\
\hline 29 & $\angle 27$ & & 147 & 84 & 146 \\
\hline 30 & 108 & & $\angle 32$ & 185 & 133 \\
\hline 32 & 82 & & $<2$ & 50 & 219 \\
\hline 32 & 20 & & 55 & 80 & $\angle 00$ \\
\hline
\end{tabular}


241-SX SLUDGE PROFILE TEMPERATURES

(S.0.P. 200.3.6)

Time $\frac{1330}{17-30.32}$

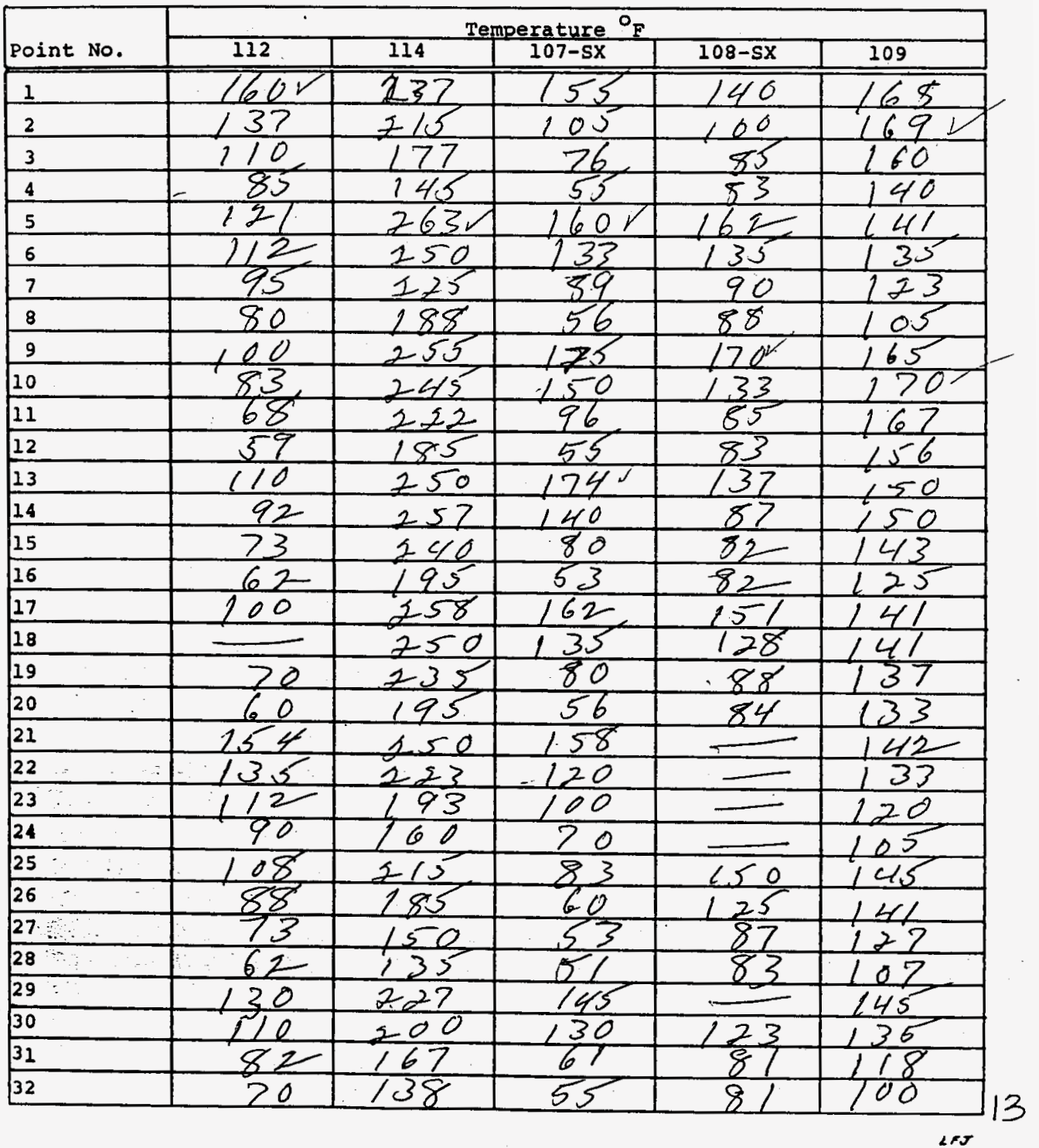


241-SX SLUDGE PROFILE TEMPERATURES

(S.O.P. 200.3.6)

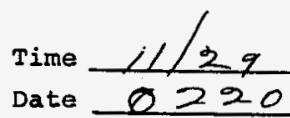

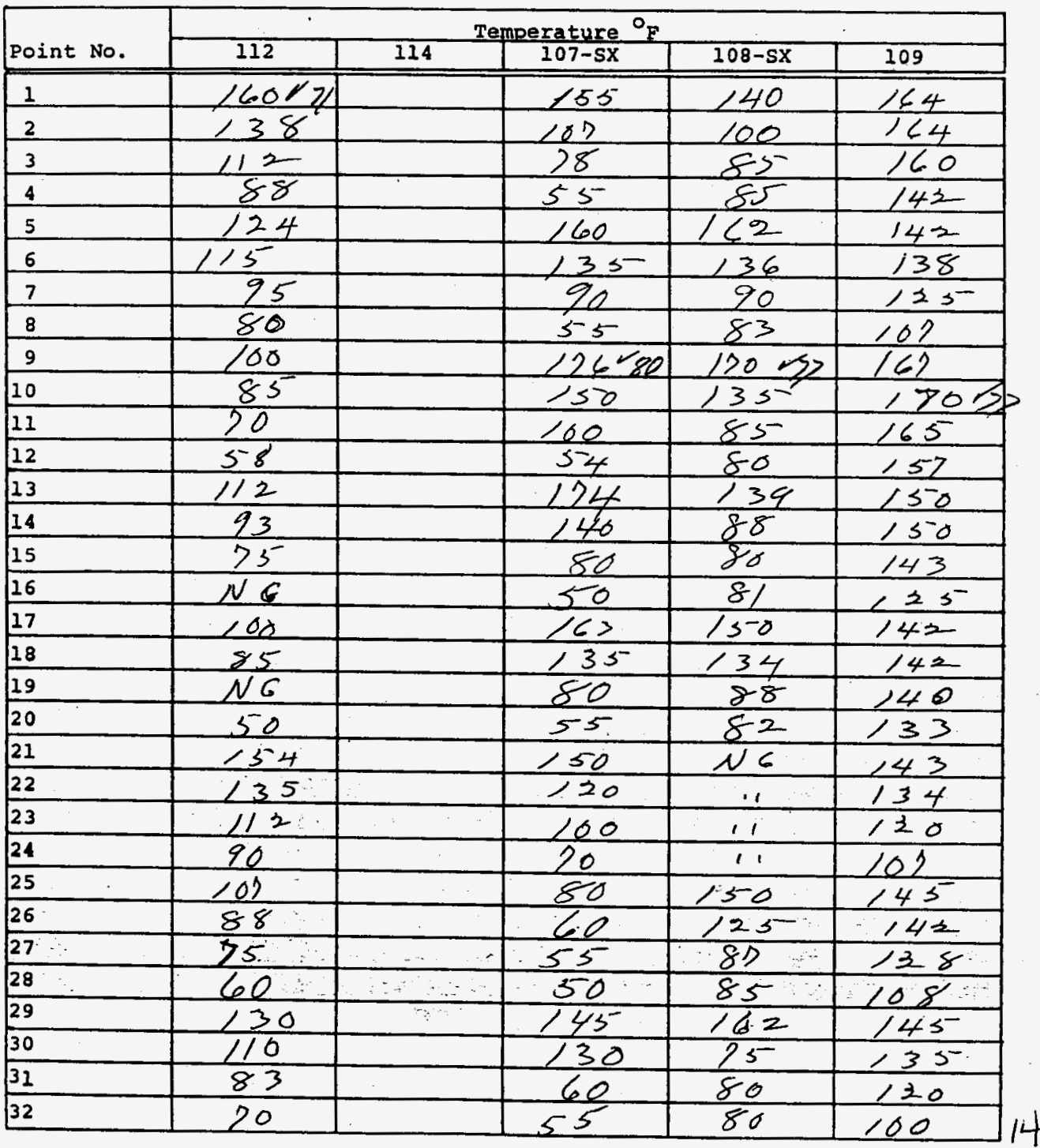




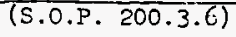

Time $\quad 11-28-72$

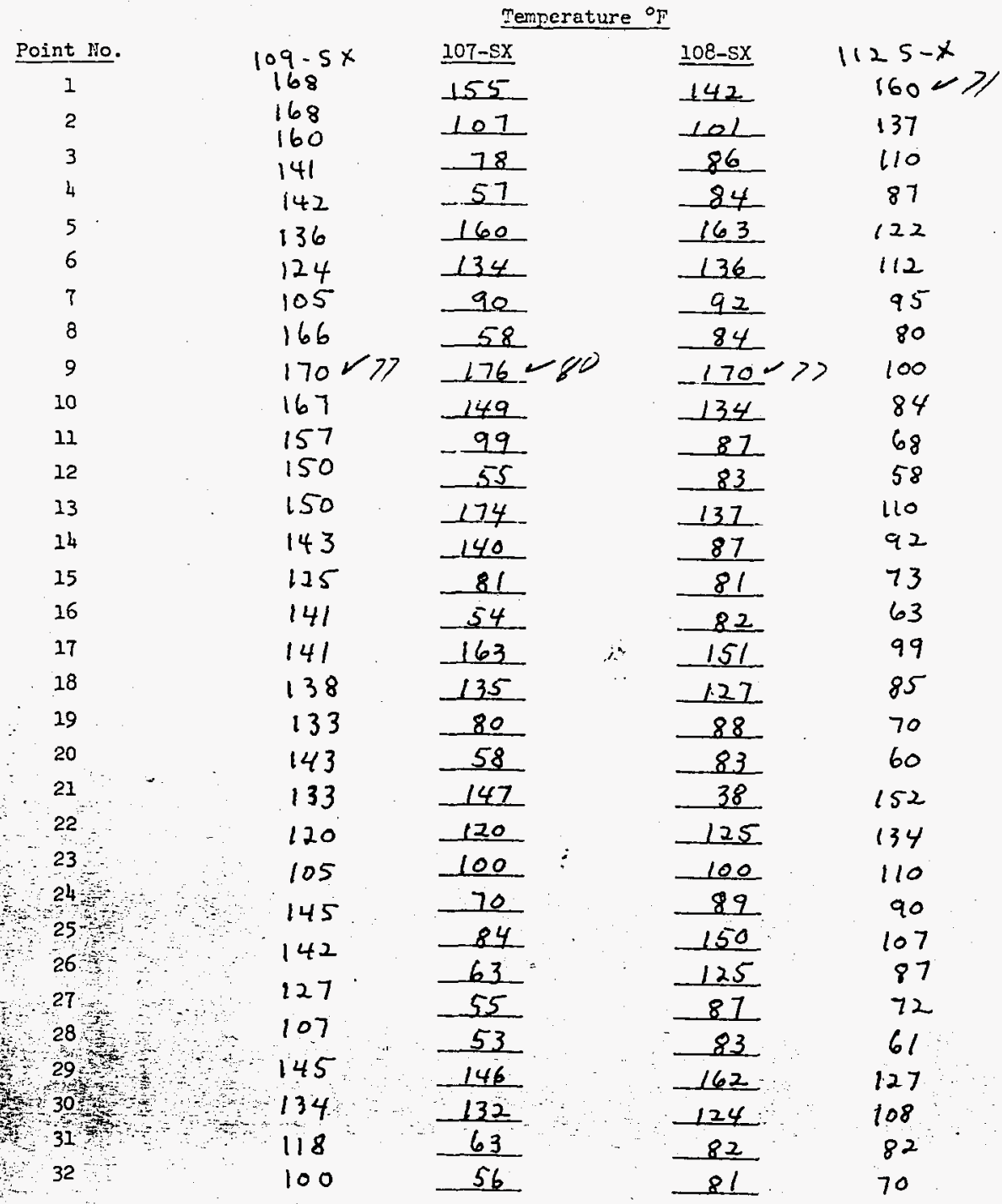


241-SX SLUDGE PROFILE TPMPERATURES

$$
\text { (5.0.P. } 200.3 .6)
$$

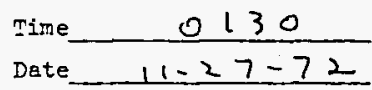

Temperature ${ }^{\circ} \mathrm{T}$

Point No.

1

2

3

4

5

6

7

8

9

10

11

12

13

14

15

16

17

18

19

20

21

22.

23

24

25

26

27

28

29

30

31

32
109

167

168

160

141

141

136

123

105

166

170.

167

158

150

150

143

125

142

142

137

133

143

133

120

105

145

142

127

107

146

135

$1 / 7$

100 107-5x

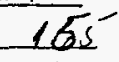

III

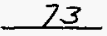

$-67$

159

133

20

57

175

148

96

55

173

$14 a$

80

55

162

135

80

57

147

120

$100:$

72

83

63.

$\frac{\sqrt{6}}{65}$

145

131

63
108-SX

140

$\frac{100}{85}$

$\frac{85}{83}$

162

$\frac{135}{90}$

$\frac{90}{83}, 95 \quad 226$

$\frac{83}{188} / 80 / 190$

$\begin{array}{ccc}133 & 75 & 246 \\ 86 & 67 & 246\end{array}$

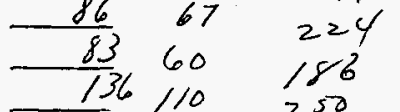

87250

256

27235

$-82 / 94$

$10100 \quad 25$

$12683 \quad 249$

$-8470 \quad 234$

8460.796

Ng/58 248.

$123 / 35 \quad 227$

$99 \quad 110 \quad 199$

1) $90 \% 169$

$150-105=215$

$123.87 \quad 187$

8672,150

83 62 135

$160 / 29 \quad 1226$

$\frac{123}{8107-202}$

80
69 
24l-SX SLUDGE PROFILE TEMPERATURES

(S.0.P. 200.3.6)

Time $\frac{1300}{11-9-72}$

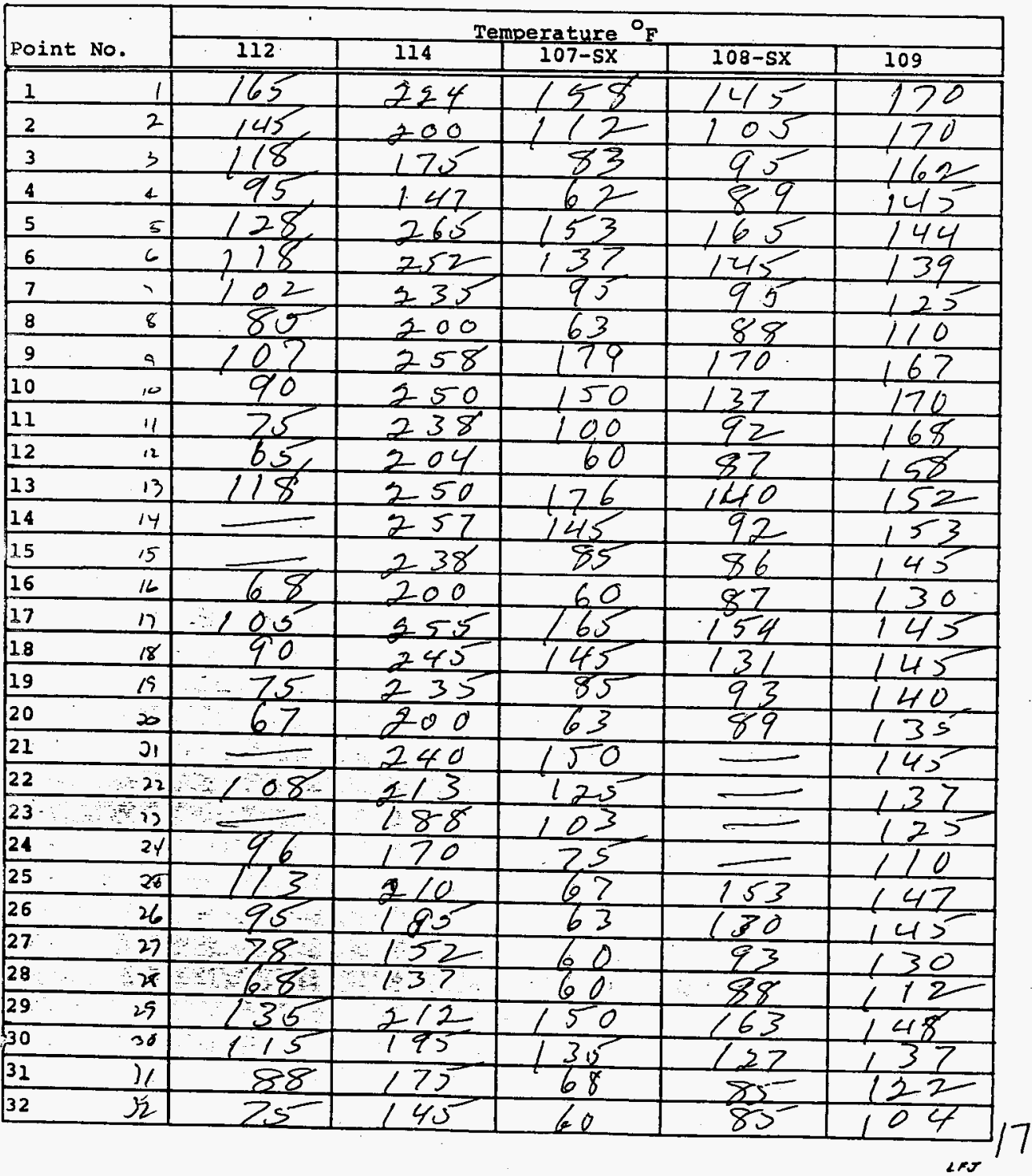


241-SX SLUDGE PROFILE TEMPERATURES

(S.O.P. 200.3.6)

Time $\frac{6900}{11-17-72}$

\begin{tabular}{|c|c|c|c|c|c|}
\hline \multirow[b]{2}{*}{ Point No. } & \multicolumn{5}{|c|}{ Temperature ${ }^{\circ} \mathrm{F}$} \\
\hline & 112 & 114 & $107-5 x$ & $108-5 x$ & 109 \\
\hline 1 & 16 & 230 & 5 & & 169 \\
\hline 2 & 14 & 207 & 11 & & 170 \\
\hline 3 & $1 / 5$ & 180 & & & 160 \\
\hline 4 & 92 & 45 & 63 & 9 & 142 \\
\hline 5 & 26 & 65 & 162 & & 142 \\
\hline 6 & 11 & 250 & 13 & & 134 \\
\hline 7 & 100 & 230 & & & 125 \\
\hline 8 & 8 & 95 & 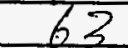 & & 108 \\
\hline 9 & & 257 & $\%$ & & 167 \\
\hline 10 & - & 250 & 150 & & 170 \\
\hline 11 & & 230 & 100 & & 168 \\
\hline 12 & & 193 & 60 & 8 & 158 \\
\hline 13 & & 250 & 175 & 44 & 150 \\
\hline 14 & & 257 & 1 & & 150 \\
\hline 15 & & 240 & & 80 & 145 \\
\hline 16 & 6 & 195 & 60 & 86 & 12 \\
\hline 17 & 6 & 253 & 164 & 152 & 142 \\
\hline 18 & 8 & 248 & & $\angle 3$ & 142 \\
\hline 19 & & 35 & & & 140 \\
\hline 20 & 6 & 98 & 63 & & \\
\hline 21 & & 245 & 150 & & 144 \\
\hline 22 & & $2 \geq 2$ & 125 & & \\
\hline 23 & & 198 & 0.3 & & 122 \\
\hline 24 & & 173 & 75 & & 107 \\
\hline 25 & 11 & 213 & 88 & 150 & 145 \\
\hline 26 & 9 & 1,87 & 68 & & 142 \\
\hline 27 & & 130 & 60 & & 130 \\
\hline 28 & $\therefore 62$ & 137 & $\therefore 60$ & 88 & 110 \\
\hline 29 & 433 & 220 & 150 & 163 & $1 \mathrm{ccs}$ \\
\hline 30 & 115 & 200 & 135 & $12^{2} 6$ & 1.37 \\
\hline 31 & 81 & 171 & 68 & 85 & 120 \\
\hline 32 & Zs & 140 & 60 & 56 & $\infty$ \\
\hline
\end{tabular}




\section{1-SX SLUDGE PROFILE TEMPERATURES}

(S.0.P. 200.3.6)

Time $\frac{0100}{11 / 3 / 72}$

\begin{tabular}{|c|c|c|c|c|c|}
\hline \multirow[b]{2}{*}{ Point No. } & \multicolumn{5}{|c|}{ Temperature ${ }^{\circ} \mathrm{F}$} \\
\hline & 112 & 114 & $107-5 x$ & $108-5 x$ & 109 \\
\hline 1 & $\angle 67$ & 222 & $\angle 59$ & 145 & 169 \\
\hline 2 & 145 & 1524 & 115 & 108 & 170 \\
\hline 3 & 118 & 132 & 86 & 95 & 163 \\
\hline 4 & 95 & 134 & 65 & 92 & 146 \\
\hline 5 & 129 & 225 & 164 & 165 & 145 \\
\hline 6 & $\angle 21$ & 265 & $<40$ & 140 & 140 \\
\hline 7 & 105 & 237 & 98 & 100 & 127 \\
\hline 8 & 81 & 202 & 65 & 91 & 112 \\
\hline 9 & $\angle O G$ & 221 & $\angle 81$ & 123 & 167 \\
\hline 10 & $\mathrm{COZ}_{2}$ & 262 & 254 & 140 & 172 \\
\hline 11 & 25 & 241 & $\angle 03$ & 96 & 169 \\
\hline 12 & 66 & 216 & 64 & 91 & $\angle 59$ \\
\hline 13 & 117 & 262 & $\angle 27 r$ & 142 & -30 \\
\hline 14 & $\angle 00$ & 213 & 146 & 94 & 151 \\
\hline 25 & $\angle Z N C$ & $243^{\circ}$ & 87 & 90 & 146 \\
\hline 16 & $N G$ & 208 & 62 & 90 & 130 \\
\hline 17 & $\angle 05$ & 270 & 167 & 155 & 144 \\
\hline 18 & 96 & 263 & 141 & 131 & 144 \\
\hline$\therefore \ldots$ & 26 & 250 & 86 & 96 & 141 \\
\hline 20 & 67 & 218 & 65 & 91 & 136 \\
\hline $21 \cdots$ & $\angle 90$ & 237 & $\angle 50$ & $N G$ & 140 \\
\hline 22 & $\angle 35$ & 201 & 125 & 11 & 136 \\
\hline 23 & 118 & $\angle 50$ & $\angle 05$ & 11 & $\angle 35$ \\
\hline 24 & 96 & $\angle 36$ & 22 & 11 & 112 \\
\hline 25 & $\angle 16$ & 208 & 88 & 152 & 146 \\
\hline 26 & 96 & 124 & 20 & 130 & 145 \\
\hline $27 \quad$ & 80 & 141 & 62 & 93 & 131 \\
\hline $28 \quad 1$ & 20 & $\because 136$ & $6 i$ & 92 & 113 \\
\hline 29. & 135 & $\angle 82$ & 150 & $\angle \angle 3$ & 149 \\
\hline 30 & 116 & $\angle 35$ & 137 & $\angle 28$ & $13 ?$ \\
\hline 31 & 90 & 135 & 20 & 90 & $1=3$ \\
\hline 32 & 26 & 135 & 63 & 90 & 106 \\
\hline
\end{tabular}


241-SX SLUDGE PROFILE TEMPERATURES

(S.0.P. 200.3.6)

Time $\frac{\angle A Q D}{\angle L-Z-12}$

\begin{tabular}{|c|c|c|c|c|c|}
\hline \multirow[b]{2}{*}{ Point No. } & \multicolumn{5}{|c|}{ Temperature ${ }^{\circ} \mathrm{F}$} \\
\hline & 112 & 114 & $107-5 x$ & $108-5 x$ & 109 \\
\hline 1 & 168 & 222 & 160 & 145 & $1>0$ \\
\hline 2 & 145 & 185 & 115 & 108 & 171 \\
\hline 3 & 120 & 135 & 85 & 93 & 163 \\
\hline 4 & 95 & 135 & 63 & 91 & 145 \\
\hline 5 & 130 & 277 & 165 & 165 & 144 \\
\hline 6 & 120 & 265 & 140 & 140 & 140 \\
\hline 7 & 104 & 239 & 96 & 100 & 127 \\
\hline 8 & 87 & 206 & 63 & 90 & 112 \\
\hline 9 & 110 & 270 & 180 & 122 & 167 \\
\hline 10 & 90 & 762 & 154 & 140 & 171 \\
\hline 11 & 75 & 241 & 103 & 94 & 168 \\
\hline 12 & 65 & 215 & 61 & 90 & 160 \\
\hline 13 & 118 & 165 & 177 & 142 & 152 \\
\hline 14 & 100 & 275 & 146 & 95 & 152 \\
\hline 15 & 80 & 245 & 87 & 89 & 145 \\
\hline 16 & 66 & 208 & 60 & 89 & 130 \\
\hline 17 & 105 & 272 & 167 & 154 & 143 \\
\hline 18 & 90 & 267 & 140 & 131 & 143 \\
\hline 19 & 75 & 245 & 85 & 95 & 140 \\
\hline 20 & 65 & 218 & 63 & 90 & 135 \\
\hline 21 & 160 & 237 & 150 & $=$ & 145 \\
\hline 22 & 100 & 201 & 125 & $\longrightarrow$ & 135 \\
\hline 23 & 118 & 150 & 105 & $=$ & 125 \\
\hline 24 & 96 & 17,5 & 75 & $\longrightarrow$ & 110 \\
\hline 25 & 115 & 208 & 89 & 154 & 146 \\
\hline 26 & 95 & 174 & 68 & 130 & 145 \\
\hline 27 & 78 & 142 & 60 & 93 & 130 \\
\hline 28 & 67 & 135 & 58 & 90 & 113 \\
\hline 29 & 130 & 176 & 157 & 165 & 150 \\
\hline 30 & 118 & 135 & 137 & 129 & 138 \\
\hline 31 & & 135 & 68 & 89 & 124 \\
\hline 32 & 76 & 135 & 60 & 90 & 107 \\
\hline
\end{tabular}


241-SX SLUDGE PROFIIE TEMPERATURES

$(5.0 . P .200 .3 .6)$

Time 2130

Date $1 /-2-72$

\begin{tabular}{|c|c|c|c|c|c|}
\hline \multirow[b]{2}{*}{ Point No. } & \multicolumn{5}{|c|}{ Temperature ${ }^{\circ} \mathrm{F}$} \\
\hline & 112 & 114 & $107-5 x$ & $108-5 x$ & 109 \\
\hline 1 & $168 \times 9$ & 221 & 160 & 145 & 169 \\
\hline 2 & 145 & 183 & 114 & 109 & 131 \\
\hline 3 & 119 & 136 & 85 & 95 & 162 \\
\hline 4 & 95 & 136 & 64 & 92 & 146 \\
\hline 5 & $\angle 29$ & 274 & 164 & $\angle 66$ & 145 \\
\hline 6 & $\angle 22$ & 265 & $\angle 40$ & $14 \mid$ & 140 \\
\hline 7 & $\angle 05$ & 238 & 98 & 100 & 126 \\
\hline 8 & 86 & 202 & 65 & 91 & 112 \\
\hline 9 & $\angle 10$ & 270 & $18115^{9}$ & $\angle 23 \sqrt[11^{4}]{ }$ & $16 ?$ \\
\hline 10 & 90 & $2<2$ & 154 & 140 & $1721^{\frac{1}{2}}$ \\
\hline 11 & 25 & 242 & $\angle 05$ & 95 & 168 \\
\hline 12 & 65 & 215 & 61 & 98 & 158 \\
\hline 13 & 119 & 265 & 127 & $\angle 43$ & $\angle 51$ \\
\hline 14 & 100 & $20510^{3}$ & 146 & 96 & $\angle 52$ \\
\hline 25 & 80 & 245 & 82 & 88 & 146 \\
\hline 16 & 68 & 208 & 60 & 90 & 130 \\
\hline 17 & 105 & 273 & $\angle 66$ & 154 & 144 \\
\hline 18 & 92 & 267 & 142 & $<3$ & 145 \\
\hline 19 & 26 & 241 & 86 & 96 & 141 \\
\hline 20 & 65 & 217 & 62 & 91 & 136 \\
\hline 21 & 162 & 237 & $\angle 50$ & $N G$ & 145 \\
\hline 22 & $N G$ & 200 & 225 & 11 & 137 \\
\hline 23 & 119 & 150 & 105 & 11 & 125 \\
\hline 24 & 99 & 138 & 26 & 11 & 112 \\
\hline 25 & 115 & 208 & 89 & $\angle 54$ & $<47$ \\
\hline 26 & 92 & 175 & 20 & 131 & 146 \\
\hline 27 & 79 & 142 & 61 & 92 & 131 \\
\hline 28 & NG & 136 & 60 & $=91$ & 115 \\
\hline 29 & 136 & 181 & $\angle 50$ & 165 & 149 \\
\hline 30 & 118 & 136 & $\angle 37$ & 130 & 138 \\
\hline 31 & 90 & 136 & 26 & 87 & 125 \\
\hline 32 & 76 & 135 & 60 & 87 & 106 \\
\hline
\end{tabular}


241-SX SLUDGE PROFILE TEMPERATURES

(S.0.P. 200.3.6)

Time $0 / 35$

Date $11 / 1 / 232 n 11$.

\begin{tabular}{|c|c|c|c|c|c|}
\hline \multirow[b]{2}{*}{ Point No. } & \multicolumn{5}{|c|}{ Temperature ${ }^{\circ} \mathrm{F}$} \\
\hline & 112 & 114 & $107-5 X$ & $108-5 x$ & 109 \\
\hline 1 & 162. & 921 & 160 & 145 & 169 \\
\hline 2 & 144 & 183 & $\angle \angle 3$ & $\angle 02$ & 170. \\
\hline 3 & 119 & $\angle 3 C$ & $133^{-}$ & 92 & $\angle 62$ \\
\hline 4 & 95 & $\angle 36$ & 62 & 90 & 146 \\
\hline 5 & 130 & 225 & $\angle 45$ & 165 & 145 \\
\hline 6 & $\angle 20$ & $2<5$ & 140 & 145 & 140 \\
\hline 7 & 101 & 238 & 98 & 99 & $\angle 26$ \\
\hline 8 & 86 & $20 \%$ & 64 & 90 & $\angle 1$ \\
\hline 9 & 110 & 272 & 180 & 122 & 162 \\
\hline 10 & 91 & 262 & 154 & $\angle 38$ & $\angle 21$ \\
\hline 11 & 35 & 240 & 104 & 92 & 168 \\
\hline 12 & 65 & 215 & 160 & 20 & $\angle 59$ \\
\hline 13 & 119 & 265 & 128. & $\angle 42$ & $\angle 53$ \\
\hline 14 & 100 & 214 & $\angle 42$ & 94 & $\angle 53$ \\
\hline 15 & 80 & 245 & 87 & 90 & 146 \\
\hline 16 & 69 & 208 & 160 & 80 & $\angle 30$ \\
\hline 17 & $\angle 06$ & 214 & 161 & $13-2$ & 145 \\
\hline 18. & 90 & 266 & 140 & $\angle 32$ & 144 \\
\hline 19 & 26 & $24 L$ & 83 & 85 & 142 \\
\hline $20=$ & 65 & $2 / 6$ & 62 & 91 & $\angle 3 C$ \\
\hline 21 & 162 & 236 & 130 & $N 6$ & 146 \\
\hline 22 & 143 & 200 & 123 & $\leftarrow$ & 136 \\
\hline 23 & 115 & 149 & $\angle 05$ & $\angle$ & 135 \\
\hline $24 \cdots$ & 99 & 132 & 26 & $\leftarrow$ & 112 \\
\hline 25 & 115 & 208 & 89 & $\angle 34$ & 147 \\
\hline 26 & 92 & 124 & 12 & $\angle 3 \%$ & 146 \\
\hline 273 & 29 & 142 & 10 & 94 & $\angle 32$ \\
\hline $28+2 \quad-$ & $\because 65$ & 135 & 59 & 90 & $\angle \angle 4$ \\
\hline $29 \quad-2$ & $\therefore \angle 35$ & 180 & $\angle 50$ & $\angle 65$ & 149 \\
\hline 30 & 115 & 135 & 137 & 28 & 139 \\
\hline 31 & 88 & 135 & 67 & 84 & $\angle 25$ \\
\hline 32 & 26 & 135 & 60 & 86 & 102 \\
\hline
\end{tabular}


241-SX SIUDGE PROFILE TEMPERATURES

(S.O.P. 200.3.6)

Time $\frac{0 / 53 / 1}{10 / 3 / 122}$

\begin{tabular}{|c|c|c|c|c|c|}
\hline \multirow[b]{2}{*}{ Point No. } & \multicolumn{5}{|c|}{ Temperature ${ }^{\circ} F$} \\
\hline & 112 & 114 & $107-5 x$ & $108-5 x$ & 109 \\
\hline 1 & 16.8 & 220 & 160 & 146 & 169 \\
\hline 2 & 145 & 182 & 115 & 107 & 120 \\
\hline 3 & $\angle 20$ & 136 & 85 & 91 & 163 \\
\hline 4 & 95 & 135 & $\operatorname{Lo}$ & 90 & 146 \\
\hline 5 & $\angle 28$ & 226 & 165 & 166 & 144 \\
\hline 6 & 124 & 265 & 140 & 142 & 140 \\
\hline 7 & 105 & 238 & 99 & 100 & 127 \\
\hline 8 & 88 & 207 & 61 & 92 & 112 \\
\hline 9 & $\angle 10$ & 220 & 181 & 173 & 167 \\
\hline 10 & 82 & 262 & 154 & 140 & 172 \\
\hline 11 & 25 & 240 & 105 & 92 & 169 \\
\hline 12 & 65 & 215 & 52 & $8 ?$ & 160 \\
\hline 13 & 119 & 266 & $\angle 28$. & 144 & 152 \\
\hline 14 & $\angle 22$ & 225 & $\angle 4\rangle$ & 23 & 154 \\
\hline 15 & 83 & 245 & 82 & $8 ?$ & 147 \\
\hline 16 & 66 & 209 & $5 ?$ & 87 & 131 \\
\hline 17 & $\operatorname{los}$ & 273 & 162 & 153 & 1444 \\
\hline 18 & 90 & $2<7$ & 142 & 132 & 144 \\
\hline 19 & 36 & 246 & 85 & 95 & 142 \\
\hline 20 & 65 & $217:$ & 60 & 90 & 136 \\
\hline 21 & $\angle 6=$ & 231 & $\angle 30$ & $N G$ & 145 \\
\hline 22 & 142 & 200 & 127 & 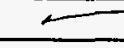 & 137 \\
\hline 23 & $\angle 37$ & 150 & 106 & $\longleftarrow$ & 125 \\
\hline 24 & $\angle 00$ & 132 & $2 c$ & $\angle$ & 112 \\
\hline 25 & 115 & 208 & 90 & 155 & $\angle 47$ \\
\hline 26 & 95 & 135 & 62 & $\angle 31$ & 145 \\
\hline 27 & 29 & 142 & 159 & 94 & 132 \\
\hline 28. & 61 & $\div 36$ & $\therefore 57$ & 90 & $1 / 5$ \\
\hline 29 & $\angle 32$ & 180 & 130 & 164 & $\angle 49$ \\
\hline 30 & 112 & 136 & $\angle 38$ & 30 & 139 \\
\hline 31 & 80 & $13 c$ & 66 & 86 & $\angle 16$ \\
\hline 32 & 75 & 135 & 57 & 84 & $\angle 07$ \\
\hline
\end{tabular}


241-SX SLUDGE PROFILE TEMPERATURES

(S.0.P. 200.3.6)

Time $\frac{0 / 50}{10 / 3 / 1 / 2}$

\begin{tabular}{|c|c|c|c|c|c|}
\hline \multirow[b]{2}{*}{ Point No. } & \multicolumn{5}{|c|}{ Temperature ${ }^{\circ}$} \\
\hline & 112 & 114 & $107-5 x$ & $108-5 x$ & 109 \\
\hline 1 & 168 & 221 & 160 & 145 & $1<9$ \\
\hline 2 & 145 & $18=$ & 115 & $\log$ & 120 \\
\hline 3 & 120 & 138 & 86 & 92 & 163 \\
\hline 4 & 100 & 136 & 11 & 91 & 145 \\
\hline 5 & $\angle 30$ & 275 & $\angle 65$ & $t \leq 1<5$ & 145 \\
\hline 6 & $\angle 24$ & $2<5$ & 140 & $\angle L 10$ & 140 \\
\hline 7 & $\angle 0 S^{\circ}$ & $240^{\circ}$ & $\angle 0 Q$ & $\cos$ & 122 \\
\hline 8 & 80 & 202 & 62 & 80 & $x=1,13$ \\
\hline 9 & 113 & 210 & $\angle 81$ & 123 & $16\rangle$ \\
\hline 10 & 94 & 263 & $\angle 55$ & 140 & $122=$ \\
\hline 11 & 22 & 242 & 105 & 83 & $1 \leq 2$ \\
\hline 22 & 65 & $2 / 5$ & 60 & 88 & 159 \\
\hline 13 & $\angle 20$ & $2<5$ & 128 & 144 & 151 \\
\hline 14 & $\angle O O$ & 275 & 148 & 85 & 154 \\
\hline 15 & 80 & 2415 & 87 & 82 & 148 \\
\hline 16 & $\Delta C$ & 210 & 58 & 82 & -32 \\
\hline 17 & $<6-7$ & 214 & 162 & 155 & 143 \\
\hline 18 & 93 & 267 & 143 & 132 & 143 \\
\hline 19 & 28 & 247 & 85 & 93 & 143 \\
\hline 20 & 68 & 217 & 62 & 83 & $\angle 32$ \\
\hline 21 & 163 & 237 & 152 & $N G$ & $\angle 4 E$ \\
\hline 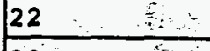 & 144 & 802 & $\angle 24$ & $\leftarrow$ & $\angle 33$ \\
\hline 23 & 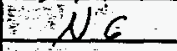 & 150 & 110 & $\leftarrow$ & 126 \\
\hline 24 & 100 & $13\rangle$ & $2 ?$ & 2 & 112 \\
\hline$\therefore: \quad$ & $\therefore / 15$ & $2 / 0$ & 90 & 255 & 148 \\
\hline $26 \ldots$ & $\angle \angle 00$ & 135 & 2.8 & 132 & 146 \\
\hline 27 & 882 & 143 & 60 & 94 & 132 \\
\hline 4 & $268^{\prime}$ & $\therefore 36$ & 58 & 80 & $\angle 15$ \\
\hline 29. & $\because 138$ & 182 & $\angle 51$ & 165 & 149 \\
\hline 30 & $1 / 20$ & 136 & 139 & 130 & 140 \\
\hline 31 & 93 & 136 & 62 & 87 & $\angle 25$ \\
\hline 32 & 80 & 135 & 60 & 87 & $\log 2$ \\
\hline
\end{tabular}


241-SX SLUDGE PROFILE TEMPERATURES

(S.0.P. 200.3.6)

Time $\frac{1340}{10-26 \cdot 72}$

\begin{tabular}{|c|c|c|c|c|c|}
\hline \multirow[b]{2}{*}{ Point No. } & \multicolumn{5}{|c|}{ Temperature ${ }^{\circ} F$} \\
\hline & 112 & 114 & $107-5 x$ & $108-5 x$ & 109 \\
\hline 1 & 169 & 220 & 160 & 146 & $1>0$ \\
\hline 2 & 147 & 183 & 115 & 120 & 120 \\
\hline 3 & 122 & 135 & 94 & 85 & 163 \\
\hline 4 & 10.0 & 135 & 70 & 94 & 146 \\
\hline 5 & 132 & 277 & 165 & 163 & 144 \\
\hline 6 & 123 & 265 & 140 & 141 & 140 \\
\hline 7 & 107 & 237 & 100 & 100 & 127 \\
\hline 8 & 91 & 207 & 70 & 93 & 112 \\
\hline 9 & 113 & 270 & 181 & 174 & 167 \\
\hline 10 & 95 & 260 & 155 & 141 & 17 \\
\hline 11 & 80 & 240 & 107 & 97 & 169 \\
\hline 12 & 71 & $2 / 3$ & 68 & 93 & 260 \\
\hline 13 & 121 & 267 & 180 & 144 & 151 \\
\hline 14 & 103 & 275 & 150 & 97 & 152 \\
\hline 15 & 83 & 240 & 90 & 92 & 145 \\
\hline 16 & 75 & 212 & 67 & 92 & 135 \\
\hline 17 & 109 & 270 & 165 & 155 & 145 \\
\hline 18 & 94 & 265 & 142 & 132 & 142 \\
\hline 19 & 80 & 245 & 90 & 96 & 140 \\
\hline 20 & 73 & 215 & 70 & 92 & 135 \\
\hline 21 & $=$ & 236 & 151 & $=$ & 145 \\
\hline 22 & 145 & 200 & 627 & $=$ & 135 \\
\hline 23 & 220 & 147 & 109 & $\longrightarrow$ & 125 \\
\hline 24 & 100 & 135 & 82 & 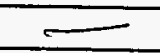 & 112 \\
\hline 25 & 119 & 207 & $=$ & 154 & 146 \\
\hline 26 & $\therefore 100$ & 174 & 73 & 1.31 & 145 \\
\hline 27 & $\therefore 83$ & 145 & 66 & 95 & 1.31 \\
\hline 28 & 75 & 135 & 65 & 92 & $1 / 5$ \\
\hline 29 & 140 & 180 & 1.52 & 165 & 150 \\
\hline 30 & 120 & 135 & 140 & 130 & 100 \\
\hline 31 & 95 & 135 & 25 & 91 & \\
\hline 32 & 81 & 135 & 68 & 92 & I \\
\hline
\end{tabular}


241-SX SLUDGE PROFILE TEMPERATURES

(S.0.P. 200.3.5)

Time 1620

Date $10-20-72$

\begin{tabular}{|c|c|c|c|c|c|}
\hline \multirow[b]{2}{*}{ Point No. } & \multicolumn{5}{|c|}{ Temperature ${ }^{\circ} \mathrm{F}$} \\
\hline & 112 & 114 & $107-5 x$ & $108-5 x$ & 109 \\
\hline 1 & 170 & 216 & 761 & 147 & 170 \\
\hline 2 & $\angle 50$ & 172 & 128 & 170 & 171 \\
\hline 3 & 125 & 135 & 90 & 97 & 163 \\
\hline 4 & 100 & 135 & 70 & 95 & 152 \\
\hline 5 & 132 & 277 & 165 & 165 & 145 \\
\hline 6 & 124 & 265 & 142 & 142 & 140 \\
\hline 7 & $=$ & 237 & 101 & 100 & 128 \\
\hline 8 & 92 & 207 & 72 & 95 & 118 \\
\hline 9 & 115 & 270 & 182 & 174 & 167 \\
\hline 10 & 95 & 255 & $\angle 55$ & 142 & 172 \\
\hline 11 & 80 & 240 & 108 & 200 & 169 \\
\hline 12 & 24 & 212 & 70 & 95 & 160 \\
\hline 13 & 123 & 267 & 180 & 145 & 152 \\
\hline 14 & 105 & 276 & 150 & 98 & 1.52 \\
\hline 15 & 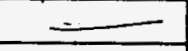 & 245 & 93 & 92 & 145 \\
\hline 16 & $=$ & 212 & 70 & 92 & 130 \\
\hline 17 & 110 & 276 & 163 & 155 & 145 \\
\hline 18 & 95 & 265 & 143 & 133 & 144 \\
\hline 19 & 82 & 242 & 91 & 97 & 140 \\
\hline 20 & 75 & 215 & 71 & 93 & 135 \\
\hline 21 & $\longleftarrow$ & 235 & 152 & $=$ & 145 \\
\hline 22 & 5 & 200 & 127 & $=$ & 135 \\
\hline 23 & 122 & 145 & 110 & $=$ & 125 \\
\hline 24 & 100 & 135 & .83 & $=$ & 112 \\
\hline 25 & $=11.8$ & 210 & 95 & 155 & 146 \\
\hline$\therefore$ & 100 & 174 & 75 & 132 & 145 \\
\hline 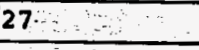 & 85 & $14 \%$ & $\therefore 68$ & 96 & 131 \\
\hline 28 & 75 & 135 & -867 & 94 & -115 \\
\hline 29 & 140 & 185 & 153 & 1 & 150 \\
\hline 30 & 126 & 13.5 & 140 & 126 & 137 \\
\hline 31 & 96.1 & 137 & 72 & 92 & 125 \\
\hline 32 & 83 & 135 & 70 & 93 & 107 \\
\hline
\end{tabular}


241-SX SLUDGE PROFILE TEMPERATURES

(S.0.P. 200.3.6)

Time $\frac{1420}{10-12-72}$

\begin{tabular}{|c|c|c|c|c|c|}
\hline \multirow[b]{2}{*}{ Point No. } & \multirow{2}{*}{\multicolumn{5}{|c|}{114 Temperature $^{\circ}{ }^{\circ}$}} \\
\hline & & & $107-5 x$ & $108-5 x$ & 109 \\
\hline 1 & 172 & 220 & $\angle 61$ & 146 & 170 \\
\hline 2 & 150 & 180 & 120 & 110 & 121 \\
\hline 3 & $125^{\circ}$ & 135 & 90 & 98 & 163 \\
\hline 4 & 105 & 135 & 25 & 95 & 148 \\
\hline 5 & 130 & 277 & 165 & 165 & 144 \\
\hline 6 & 125 & 265 & 143 & 143 & 140 \\
\hline 7 & $\angle 10$ & 235 & 103 & 103 & 127 \\
\hline 8 & 95 & 205 & 25 & 95 & 145 \\
\hline 9 & 115 & $2>0$ & 183 & 124 & 162 \\
\hline 10 & $\angle 00$ & 260 & 156 & 14 & 171 \\
\hline 11 & 83 & 235 & $\angle \angle C$ & 100 & 168 \\
\hline 12 & 75 & $2 / 2$ & 20 & 95 & 160 \\
\hline 13 & 125 & 270 & $\angle 80$ & $145^{-}$ & 150 \\
\hline 14 & 106 & 272 & 150 & 100 & 152 \\
\hline 15 & 87 & 247 & 95 & 92 & 146 \\
\hline 16 & 79 & $2 / 5$ & 20 & 92 & 130 \\
\hline 17 & 113 & 271 & $\angle 68$ & 136 & 145 \\
\hline 18 & E7 & 267 & $\angle C 5$ & 133 & 144 \\
\hline 19 & 83 & 240 & 92 & 96 & 140 \\
\hline 20 & 26 & $z / z$ & 25 & 93 & 135 \\
\hline $21 \ldots$ & 168 & 3,29 & 152 & KG & 144 \\
\hline $22-4, \quad$ & +150 & $\angle 90$ & 130 & $\angle Y G$ & 135 \\
\hline 23 & $4 / 25$ & 140 & $\angle \angle O$ & 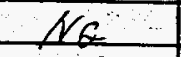 & 125 \\
\hline $24 \quad 5$ & $=/ 0 s^{\circ}$ & 135 & 85 & $\angle C$ & $\angle 13$ \\
\hline 25 & $\angle 20$ & $\therefore 210$ & $A K E$ & 155 & $115^{-}$ \\
\hline $26 \quad 02$ & $\therefore \angle O 2$ & 174 & $25-$ & 133 & 145 \\
\hline 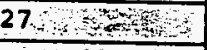 & $\alpha 8=855$ & 1445 & 20 & 98 & $13 z$ \\
\hline $28-4 b+2$ & $32 x+77=$ & $<35$ & $\because 68$ & 94 & $1 / 5$ \\
\hline $29 \quad 5$ & $7 / 45^{-1}$ & $187^{\circ}$ & 155 & $N G$ & 150 \\
\hline 30 & 125 & $\angle 35$ & 146 & $\angle 30$ & 125 \\
\hline$\therefore$ & $\therefore \angle 00$ & 135 & 80 & 92 & $1<2$ \\
\hline 32 & 85 & 1,25 & 23 & 93 & $\angle C$. \\
\hline
\end{tabular}


241-SX SLUDGE PROFILE TEMPERATURES

(S.0.P. 200.3 .6$)$

Time $\frac{1430}{10-5-22}$

\begin{tabular}{|c|c|c|c|c|c|}
\hline \multirow[b]{2}{*}{ Point No. } & \multicolumn{5}{|c|}{ Temperature ${ }^{\circ} F$} \\
\hline & 112 & 114 & $107-\mathrm{sx}$ & $108-5 x$ & 10.9 \\
\hline 1 & 170 & 220 & 162 & 147 & 170 \\
\hline 2 & 150 & 180 & 120 & 112 & 171 \\
\hline 3 & 128 & 135 & 97 & 100 & 163 \\
\hline 4 & 107 & 135 & 75 & 95 & 147 \\
\hline 5 & 135 & 27 & 165 & 165 & 145 \\
\hline 6 & 125 & 265 & 145 & 142 & 140 \\
\hline 7 & 110 & 235 & 105 & 92 & 130 \\
\hline 8 & 95 & 207 & 75 & 95 & 115 \\
\hline 9 & 117 & 267 & 185 & 175 & 167 \\
\hline 10 & 100 & 258 & 157 & 142 & 170 \\
\hline 11 & 95 & 235 & 110 & 100 & 169 \\
\hline 12 & 72 & 207 & 75 & 95 & 160 \\
\hline 13 & 127 & 268 & 180 & 145 & 151 \\
\hline 14 & 107 & 272 & 150 & 100 & 152 \\
\hline 15 & 89 & 250 & 95 & 93 & 146 \\
\hline 16 & $=$ & 217 & 75 & 93 & 131 \\
\hline 17 & 113 & 270 & 170 & 155 & 145 \\
\hline 18 & 100 & 260 & 145 & 133 & 144 \\
\hline 19 & 85 & 737 & 95 & 97 & 140 \\
\hline 20 & 77 & 210 & 75 & 93 & 135 \\
\hline 21 & 100 & 225 & $=$ & $=$ & 145 \\
\hline 22 & 150 & 200 & 130 & $=$ & 137 \\
\hline 23 & 125 & 140 & 117 & $=$ & 126 \\
\hline 24 & 105 & 135 & 85 & $=$ & 115 \\
\hline 25 & 125 & 210 & 97 & 155 & 146 \\
\hline 26 & 103 & 175 & 79 & 132 & 145 \\
\hline 27 & 85 & 140 & 20 & & 133 \\
\hline 28 & 80 & 135 & 70 & 93 & 115 \\
\hline 29 & 145 & 190 & 155 & 165 & 150 \\
\hline 30 & 120 & 135 & 142 & 131 & 140 \\
\hline 32 & 100 & 135 & 486 & $* 93$ & 125 \\
\hline 32 & 80 & 135 & 75 & 94 & 110 \\
\hline
\end{tabular}


241-SX SLUDGE PROFILE TEMPERATURES

(S.O.P. 200.3.6)

Time $\frac{0 / 00}{10 / 6 / 22 \pi / 1}$

\begin{tabular}{|c|c|c|c|c|c|}
\hline \multirow[b]{2}{*}{ Point No. } & \multicolumn{5}{|c|}{ Temperature ${ }^{\circ} \mathrm{F}$} \\
\hline & 112 & 114 & $107-5 x$ & $108-5 x$ & 109 \\
\hline 1 & 123 & 221 & 163 & 149 & 170 \\
\hline 2 & $15-0$ & 155 & 121 & 113 & 121 \\
\hline 3 & $1 \geq 9$ & 136 & 94 & 100 & 164 \\
\hline 4 & 112 & 135 & 23 & gq & 149 \\
\hline 5 & 135 & $2 ? 7$ & 166 & $162 \sqrt{16}$ & 145 \\
\hline 6 & 122 & $2<5$ & 144 & 144 & 142 \\
\hline 7 & 112 & 237 & 108 & $\angle 03$ & 130 \\
\hline 8 & 100 & 206 & 25 & 85 & 115 \\
\hline 9 & 118 & 270 & $183^{\circ}$ & 175 & 162 \\
\hline 10 & 100 & 258 & 159 & 144 & 1721 \\
\hline 11 & $8 \div 4$ & 236 & 112 & 100 & $\angle 69$ \\
\hline 12 & 75 & 208 & 166 & 83 & 160 \\
\hline 13 & 125 & 270 & $\angle 81$ & 146 & 152 \\
\hline 14 & 110 & 278 & $\angle 51$ & 89 & 154 \\
\hline 15 & 92 & 250 & 96 & 23 & 148 \\
\hline 16 & 21 & 212 & $\angle 8$ & 43 & 132 \\
\hline 17 & 115 & 211 & 169 & 156 & 144 \\
\hline 18 & 100 & 260 & 146 & $\angle 35$ & 145 \\
\hline 19 & 85 & 238 & 85 & 100 & 143 \\
\hline 20 & 25 & $2 / 2$ & 13 & 96 & 136 \\
\hline 21 & 168 & 232 & $\sqrt{6}$ & $\sqrt{6}$ & 146 \\
\hline 22 & $\angle 50$ & 195 & .1 & .1 & 138 \\
\hline 23 & 125 & 140 & 114 & 11 & 122 \\
\hline 24 & 105 & $\angle 36$ & 86 & 11 & 113 \\
\hline 25 & 125 & 212 & $\Delta / 6$ & 155 & 148 \\
\hline 26 & 106 & 175 & 27 & 135 & 142 \\
\hline 27 & 87 & $\angle 42$ & 21 & 98 & 134 \\
\hline 28 & 175 & 135 & 20 & 96 & 117 \\
\hline 29 & 145 & 181 & 155 & $\angle C L$ & 150 \\
\hline 30 & 127 & $\angle 3 C$ & 142 & 132 & 140 \\
\hline 31 & 100 & 136 & 77 & 82 & $12 c$ \\
\hline 32 & 86 & 135 & 21 & 82 & 112 \\
\hline
\end{tabular}


241-SX SLUDGE PROFILE TEMPERATURES

(S.O.P. 200.3.6)

Time $\frac{0040}{10 / 5 / 22}$

\begin{tabular}{|c|c|c|c|c|c|}
\hline \multirow[b]{2}{*}{ Point No. } & \multicolumn{5}{|c|}{ Temperature ${ }^{\circ} \mathrm{F}$} \\
\hline & 112 & 114 & $107-\mathrm{SX}$ & $108-5 x$ & 109 \\
\hline 1 & 172 & 220 & $\angle C 2$ & 148 & 120 \\
\hline 2 & 150 & 180 & $\angle 20$ & 115 & 170 \\
\hline 3 & 117 & 135 & 93 & $\angle 0 O$ & 27163 \\
\hline 4 & $10\rangle$ & 135 & 25 & 92 & 149 \\
\hline 5 & $\angle 3 s^{-}$ & 222 & $\angle 67$ & 162 & 145 \\
\hline 6 & $\angle \geq 6$ & 265 & $\angle 45$ & $\angle 45$ & 142 \\
\hline 7 & 110 & 232 & $\angle 05$ & 103 & 130 \\
\hline 8 & 95 & 205 & 25 & 95 & 116 \\
\hline 9 & 112 & 270 & 183 & 125 & 168 \\
\hline 10 & $\angle 00$ & 258 & $\angle 58$ & 145 & 122 \\
\hline 1,1 & 85 & 235 & $\angle 12$ & $\angle 0 \sigma$ & 169 \\
\hline 12 & 25 & 208 & 22 & 95 & 161 \\
\hline 13 & $125^{-}$ & 270 & 181 & 145 & 151 \\
\hline 14 & $\angle 08$ & 277 & 151 & 100 & 155 \\
\hline 15 & 89 & 250 & 89 & 25 & 149 \\
\hline 16 & 29 & 212 & 23 & 96 & 133 \\
\hline 17 & $\angle 10$ & 221 & $\angle 69$ & 152 & 145 \\
\hline 18 & $\angle 00$ & 261 & $\angle 46$ & 135 & 145 \\
\hline 19 & 85 & 238 & 25 & 100 & 143 \\
\hline 20 & 25 & 211 & 25 & 94 & 177 \\
\hline 21 & 168 & 732 & $\angle 54$ & $1 V C$ & 146 \\
\hline 22 & 200 & 196 & $\angle 30$ & 21 & $\angle 40$ \\
\hline 23 & 125 & 140 & 115 & 11 & 127 \\
\hline 24 & 105 & $\angle 35$ & 86 & 11 & 116 \\
\hline 25 & 125 & $2 / 2$ & 99 & 156 & 148 \\
\hline 26 & $\angle 05$ & 125 & 80 & 134 & 146 \\
\hline 27 & 87 & 143 & 23 & 100 & 135 \\
\hline 28 & 77 & 135 & 22 & 85 & 112 \\
\hline 29 & $\angle 44$ & 188 & 155 & 166 & 150 \\
\hline 30 & 125 & 136 & 142 & 132 & 140 \\
\hline 31 & $\angle 00$ & 136 & 81 & 85 & 126 \\
\hline 32 & 86 & $\angle 3<$ & 25 & 95 & 112 \\
\hline
\end{tabular}


241-SX SLUDGE PROFILE TEMPERATURES

(S.O.P. 200.3.6)

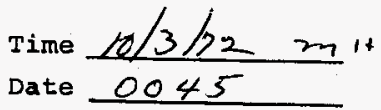

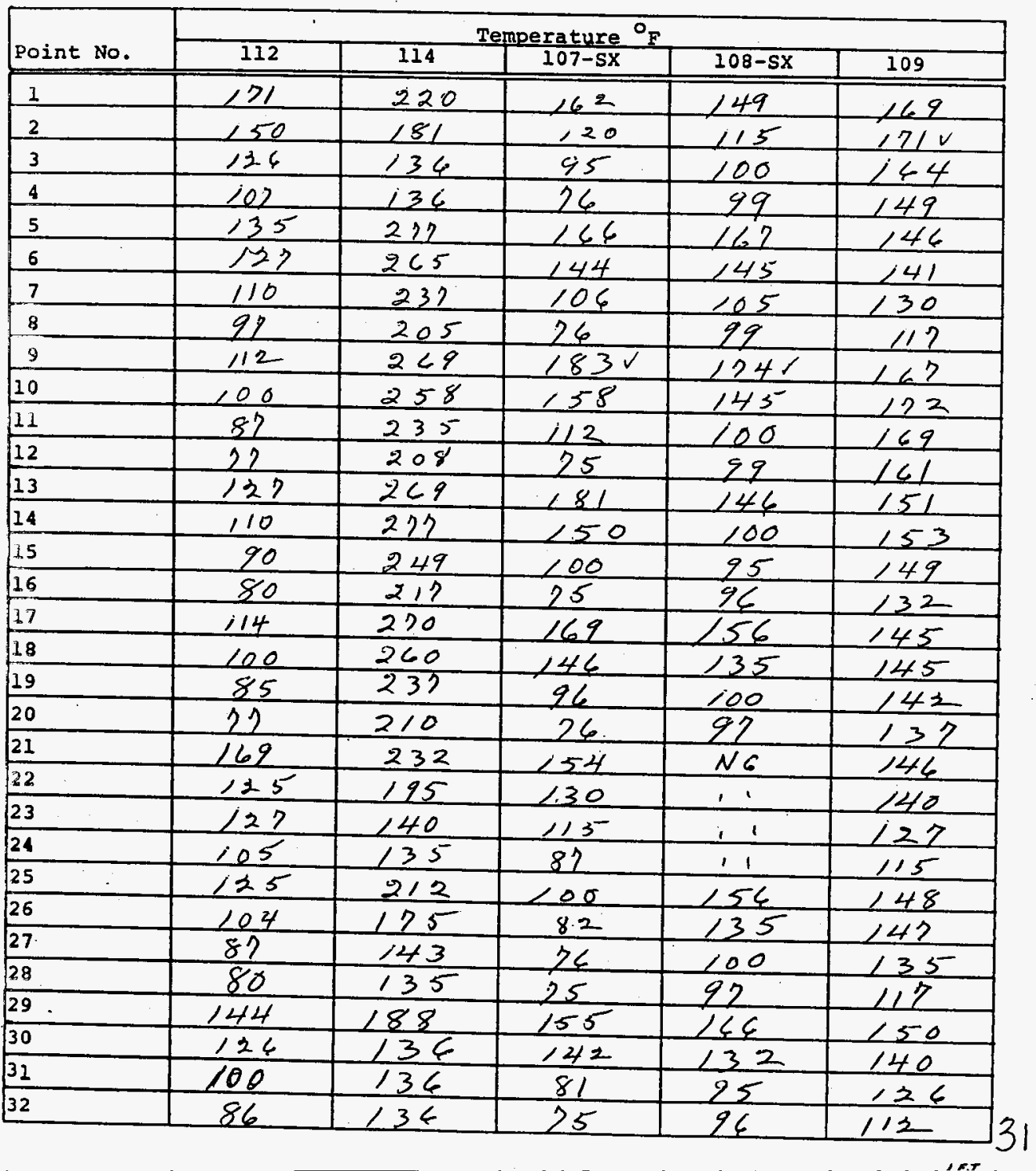


241-SX SIUDGE PROFILE TEMPERATURES

(S.0.P. 200.3.6)

Time $\frac{0 / 20}{10 / 3 / 22} \frac{11}{m a t e}$

\begin{tabular}{|c|c|c|c|c|c|}
\hline \multirow[b]{2}{*}{ Point No. } & \multicolumn{5}{|c|}{ Temperature ${ }^{\circ} \mathbf{F}$} \\
\hline & 112 & 114 & $107-5 x$ & $208-5 x$ & 109 \\
\hline 1 & 170 & 220 & 163 & 149 & 176 \\
\hline 2 & 150 & $\angle 80$ & 120 & 114 & 121 \\
\hline 3 & 128 & 1.35 & 95 & $\angle 0 O$ & $1<4$ \\
\hline 4 & 102 & 135 & 25 & 99 & 149 \\
\hline 5 & 1.36 & 277 & 162 & 167 & 145 \\
\hline 6 & 127 & 265 & 145 & 145 & 141 \\
\hline 7 & 110 & 237 & 102 & $\angle 05$ & 130 \\
\hline 8 & 95 & 205 & 25 & 99 & 115 \\
\hline 9 & 118 & 268 & $\angle 84^{\prime}$ & $175 v$ & 168 \\
\hline 10 & $\angle 0 Q$ & 258 & 158 & 146 & 171. \\
\hline 11 & 85 & 235 & $\angle \angle 3$ & 100 & 169 \\
\hline 12 & 75 & 202 & 23 & 42 & 160 \\
\hline 13 & 126 & 270 & 281 & 146 & $13^{-2}$ \\
\hline 14 & 108 & 277 & 150 & 100 & $13-4$ \\
\hline 15 & 90 & 250 & 89 & 86 & 149 \\
\hline 16 & 80 & 217 & 23 & q3 & 132 \\
\hline 17 & 115 & 120 & 169 & 157 & 145 \\
\hline 19 & 100 & 260 & 146 & $13 c$ & 243 \\
\hline 19 & 85 & 232 & 97 & $\angle 00$ & 143 \\
\hline 20 & 27 & 212 & 25 & 91 & $13>$ \\
\hline 21 & 120 & 232 & 155 & $N C$ & 146 \\
\hline 22 & 160 & 195 & 130 & $.1 \quad 1$ & 139 \\
\hline 23 & 126 & 140 & 115 & 1,1 & 124 \\
\hline 24 & $\angle O C$ & 136 & 88 & 11.1 & 115 \\
\hline 25 & 123 & 212 & 29 & 155 & 148 \\
\hline 26 & 105 & 116 & 81 & 134 & $14 c$ \\
\hline 27 & 85 & $\angle 43$ & 75 & 99 & 135 \\
\hline 28 & 78 & $\angle 35$ & 24 & 95 & 117 \\
\hline 29 & 150 & $\angle 88$ & $\angle 56$ & $1<5$ & $\angle 50$ \\
\hline 30 & $\angle 25$ & $\angle 36$ & 143 & 127 & 140 \\
\hline 31 & 100 & 135 & 80 & 95 & 125 \\
\hline 32 & 85 & 135 & 73 & 95 & 112 \\
\hline
\end{tabular}


241-SX SLUDGE PROFILE TEMPERATURES

(S.0.P. 200.3.6)

Time $\frac{0120}{10 / 2 / 12 \text { mate }}$

\begin{tabular}{|c|c|c|c|c|c|}
\hline \multirow[b]{2}{*}{ Point No. } & \multicolumn{5}{|c|}{ Temperature ${ }^{\circ} \mathrm{F}$} \\
\hline & 112 & 114 & $107-5 x$ & $108-S X$ & 109 \\
\hline 1 & $120 \sqrt{67}$ & 220 & 162 & 148 & 169 \\
\hline 2 & 150 & 180 & $\angle 20$ & 214 & 169 \\
\hline 3 & $<26$ & 135 & 95 & $\angle 00$ & 163 \\
\hline 4 & $\therefore 45$ & $\angle 34$ & 26 & $\angle 00$ & 149 \\
\hline 5 & 135 & 227 & 167 & 167 & 145 \\
\hline 6 & $\angle 27$ & 265 & 144 & $1-44$ & $|4|$ \\
\hline 7 & $\therefore 1$ & $23 c$ & 105 & 104 & 130 \\
\hline 8 & 86 & 205 & 36 & 95 & 116 \\
\hline 9 & $1<7$ & $2<8$ & $6<84$ & $174 \sqrt{69}$ & $1<7$ \\
\hline 10 & 100 & 259 & 157 & 144 & $102 \sqrt{68}$ \\
\hline 11 & 85 & 235 & 111 & 101 & 169 \\
\hline 12 & 25 & 2 ol & 125 & 92 & 160 \\
\hline 13 & $\angle 37$ & 230 & $\angle 81$ & $\angle 45$ & $\angle 50$ \\
\hline 14 & 110 & 212 & $\mid 51$ & 100 & 154 \\
\hline 15 & 90 & 250 & 99 & 96 & 149 \\
\hline 16 & 82 & 217 & 25 & 45 & 133 \\
\hline 17 & 115 & 220 & 169 & 156 & 145 \\
\hline 18 & $\angle 00$ & 260 & $\angle 46$ & 135 & 145 \\
\hline 19 & 86 & 237 & 99 & 100 & 144 \\
\hline 20 & 29 & 210 & 26 & 96 & 140 \\
\hline 21 & 170 & 232 & $\angle 5.5$ & $\sqrt{G}$ & 146 \\
\hline 22 & 150 & 195 & 130 & $x-x$ & $\angle 39$ \\
\hline 23 & 125 & 140 & 115 & $+x$ & 128 \\
\hline 24 & 105 & 130 & 89 & $x x$ & 117 \\
\hline 25 & $\angle 24$ & 212 & 99 & 155 & 148 \\
\hline 26 & 203 & 125 & 82 & 134 & 146 \\
\hline 27 & 82 & 143 & 26 & 100 & $\angle 35$ \\
\hline 28 & 25 & $\angle 32$ & 25 & 96 & $\angle 18$ \\
\hline 29 & $N C$ & 188 & $\angle 55$ & 165 & 150 \\
\hline 30 & $\angle 25$ & $13 c$ & $\angle 42$ & 132 & $\angle 40$ \\
\hline 31 & $\angle 00$ & 135 & 77 & 95 & 136 \\
\hline 32 & 85 & $\angle 34$ & 75 & 96 & $\angle \angle 2$ \\
\hline
\end{tabular}


241-SX SLUDGE PROFILE TEMPERATURES

(S.0.P. 200.3.6)

Time $\frac{1045}{9-29-72}$

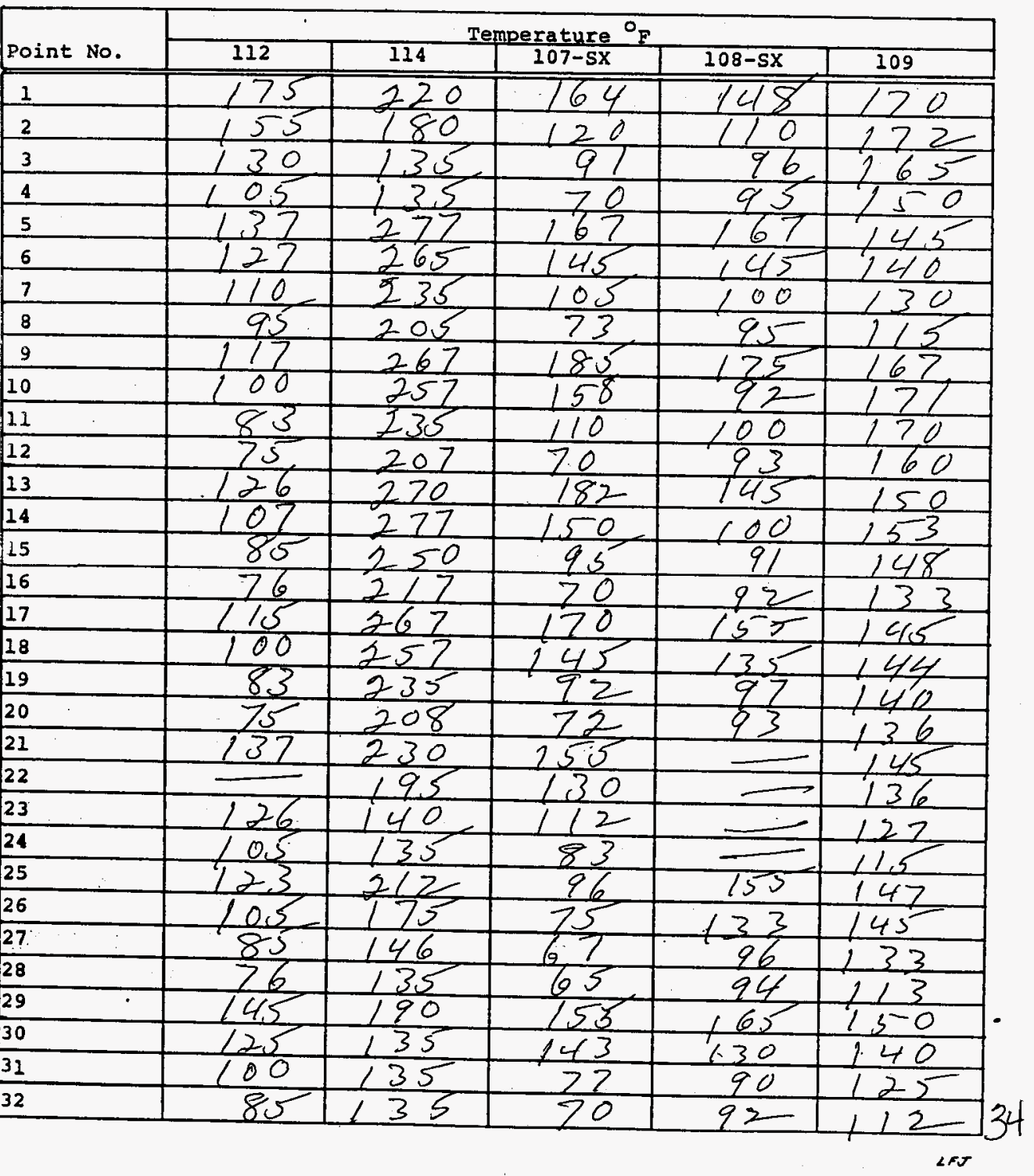


241-SX SLUDGE PROFIIE TEMPERATURES

(S.0.P. 200.3.6)

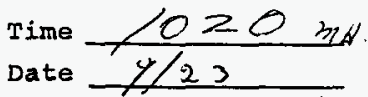

\begin{tabular}{|c|c|c|c|c|c|}
\hline \multirow{2}{*}{ Point No. } & \multicolumn{5}{|c|}{ Temperature ${ }^{\circ} \mathrm{F}$} \\
\hline & 112 & 114 & $107-5 x$ & $108-5 x$ & 109 \\
\hline 1 & 121 & 218 & 163 & 150 & 169 \\
\hline 2 & $\angle \cos$ & $\angle S O$ & $\angle 25$ & 115 & 112 \\
\hline 3 & 137 & 135 & 45 & 100 & $1<5$ \\
\hline 4 & $\angle 0 ?$ & 135 & 25 & 99 & 149 \\
\hline 5 & $\angle 39$ & 278 & $\langle 6\rangle$ & 167 & 145 \\
\hline 6 & 124 & $2<5$ & 148 & 146 & 143 \\
\hline 7 & 115 & 235 & 110 & $\angle 05$ & 132 \\
\hline 8 & $\operatorname{Los}$ & 202 & 25 & 97 & 118 \\
\hline 9 & 120 & $2<7$ & $\angle 84$ & 125 & 167 \\
\hline 10 & $\angle 22$ & 253 & 160 & $\angle 45$ & $\angle 22$ \\
\hline 11 & 56 & 233 & 115 & 100 & 120 \\
\hline 12 & $2 C$ & 205 & 25 & 88 & $\angle \leq 2$ \\
\hline 13 & $\angle 30$ & 262 & 183 & $\langle 4\rangle$ & 153 \\
\hline 14 & 112 & 228 & $<54$ & $\angle 00$ & 134 \\
\hline 15 & 20 & 250 & 100 & 87 & 148 \\
\hline 16 & 50 & 220 & 25 & 91 & $\angle 35$ \\
\hline 17 & 119 & $2<8$ & $\angle 20$ & $<57$ & 146 \\
\hline 18 & 105 & 258 & 149 & $\angle 35$ & 145 \\
\hline 19 & 88 & 2.35 & 100 & $\angle A B$ & $\angle 43$ \\
\hline 20 & 80 & 208 & 12616 & $9<$ & $\angle 38$ \\
\hline 21 & 174 & 2.30 & $<55$ & $\Delta C$ & 142 \\
\hline 22 & $\angle 55$ & 123 & $\angle 32$ & 11 & $\angle 40$ \\
\hline 23 & $\angle 30$ & $\angle 40$ & $\angle \angle 6$ & 11 & $\angle 30$ \\
\hline 24 & $\angle \angle O$ & 136 & 86 & 11 & 118 \\
\hline 25 & 126 & 213 & $N C$ & $\angle 5 C$ & 149 \\
\hline 26 & 209 & 121 & 22 & $\angle 34$ & $\angle 42$ \\
\hline 27. & 21 & $\angle 42$ & 20 & $\cos$ & $\angle 35$ \\
\hline 28 & 81 & $\angle 40$ & 69 & 28 & $\angle 20$ \\
\hline 29 & $\angle 50$ & 188 & $\angle 52$ & 167 & $\angle 50$ \\
\hline 30 & 130 & 136 & $\angle 45$ & $\angle 32$ & 140 \\
\hline 32 & $\angle 10$ & 135 & 80 & 95 & 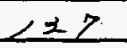 \\
\hline 32 & 86 & $\angle 35$ & 25 & 95 & 114 \\
\hline
\end{tabular}


241-SX SLUDGE PROFILE TEMPERATURES

(S.O.P. 200.3.6)

Time 1346

Date 9.21 .2

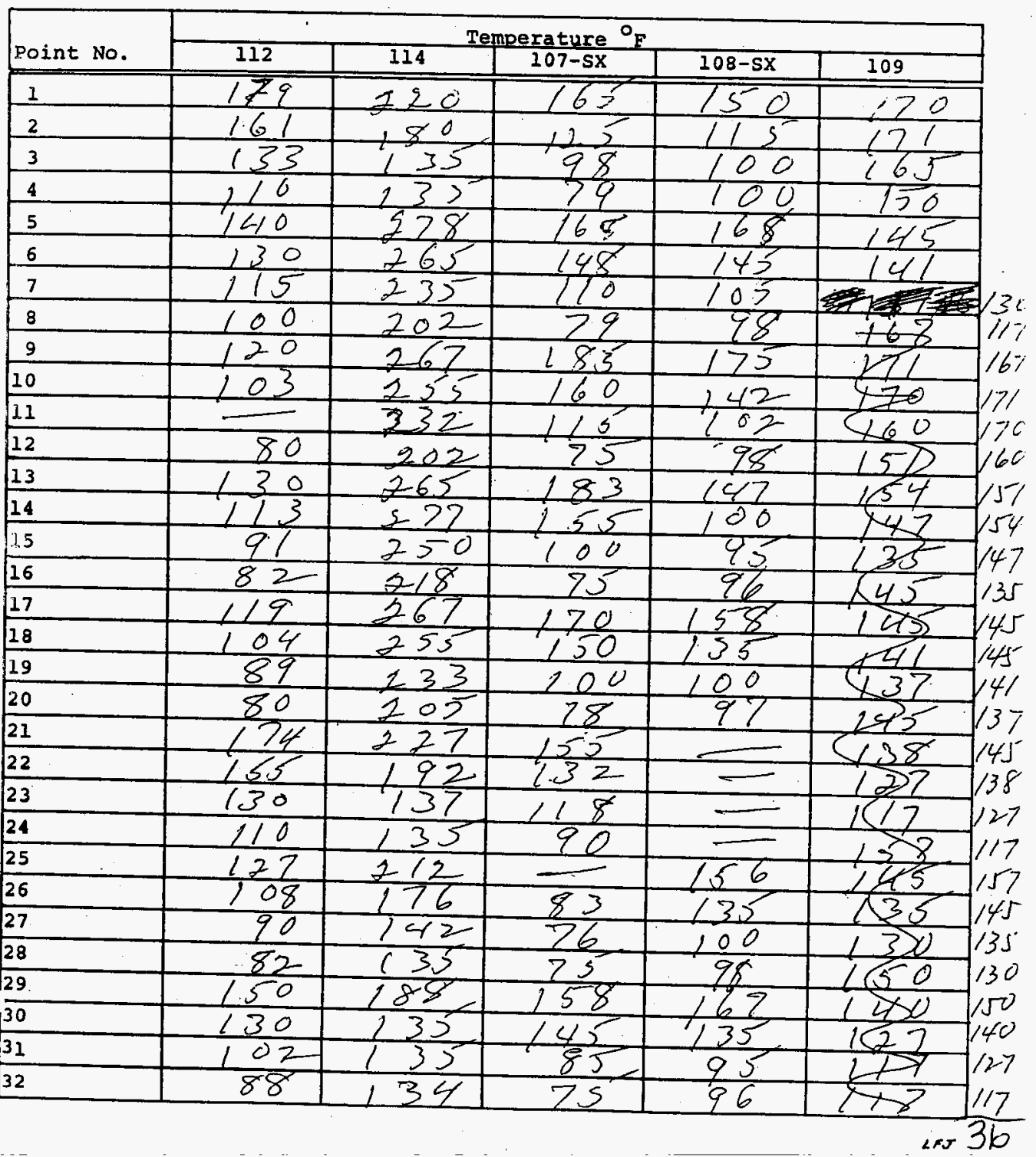


241-SX SLUDGE PROFILE TEMPERATURES

(S.0.P. 200.3.6)

Time $\frac{1330}{9-13-2-2}$

\begin{tabular}{|c|c|c|c|c|c|}
\hline \multirow[b]{2}{*}{ Point No. } & \multicolumn{5}{|c|}{ Temperature ${ }^{\circ} \mathrm{F}$} \\
\hline & 112 & 114 & $107-5 x$ & $108-5 x$ & 109 \\
\hline 1 & 180 & 215 & 165 & 150 & 170 \\
\hline 2 & 152 & 175 & 125 & 5 & \\
\hline 3 & 135 & 135 & 200 & 101 & 165 \\
\hline 4 & 112 & 135 & 82 & 100 & 150 \\
\hline 5 & 140 & 278 & 168 & 168 & \\
\hline 6 & 130 & 265 & 149 & 143 & 140 \\
\hline 7 & 115 & 235 & 110 & & \\
\hline 8 & 1.00 & 200 & & & \\
\hline 9 & 123 & 260 & 185 & & \\
\hline 10 & 105 & 255 & 160 & 12 & \\
\hline 11 & 89 & 230 & 176 & 104 & 169 \\
\hline 12 & 82 & 200 & 80 & 200 & 160 \\
\hline 13 & 122 & 272 & 184 & 147 & 151 \\
\hline 14 & 113 & 280 & 155 & 103 & 152 \\
\hline 15 & 93 & 250 & 101 & & 146 \\
\hline 16 & 85 & 218 & 80 & & 134 \\
\hline 17 & 120 & 265 & 120 & 1 & 145 \\
\hline 18 & 105 & 2.54 & 1.50 & & 144 \\
\hline 19 & 90 & 230 & 100 & 0 & 140 \\
\hline 20 & 83 & 202 & 81 & & \\
\hline 21 & 275 & 225 & 155 & & 14 \\
\hline 22 & 155 & 180 & 133 & & 137 \\
\hline 23 & - & 135 & 118 & & 127 \\
\hline 24 & 112 & $\angle 35$ & $\phi$ & & 117 \\
\hline 25 & 1.30 & 712 & 102 & & 4 \\
\hline 26 & 110 & 126 & .83 & & 142 \\
\hline 27 & 92 & 140 & 76 & 100 & 138 \\
\hline 28 & 85 & & 75 & & 119 \\
\hline 29 & 150 & 180 & 15 & 16 & 150 \\
\hline 30 & 132 & 135 & 145 & 13 & 140 \\
\hline 32 & 105 & 135 & 86 & & 126 \\
\hline 32 & 90 & 10.5 & 81 & 98 & 116 \\
\hline
\end{tabular}


24l-SX SLUDGE PROFILE TEMPERATURES

(S.0.P. 200.3.6)

Time $\frac{0 / 20}{4 / 8 / 92}$

\begin{tabular}{|c|c|c|c|c|c|}
\hline \multirow[b]{2}{*}{ Point No. } & \multicolumn{5}{|c|}{ Temperature ${ }^{\circ} F$} \\
\hline & 112 & 114 & $107-5 x$ & $108-5 x$ & 109 \\
\hline 1 & $279 \mathrm{ron}$ & $2 / 3$ & 165 & $<50$ & 169 \\
\hline 2 & $\angle 62$ & 169 & $\angle 26$ & 112 & 169 \\
\hline 3 & 137 & $\angle 34$ & $\angle 01$ & 103 & 164 \\
\hline 4 & 114 & 134 & 82 & 100 & 150 \\
\hline 5 & 142 & 228 & $\angle 68$ & 168 & $<45$ \\
\hline 6 & $\angle 33$ & 263 & 149 & 142 & 141 \\
\hline 7 & 118 & 233 & 114 & $\angle 08$ & 131 \\
\hline 8 & 103 & 200 & 83 & 100 & 118 \\
\hline 9 & $\angle 25$ & 265 & $\angle 8570$ & 12628 & 167 \\
\hline 10 & 108 & 252 & $\angle C 1$ & 147 & $221>2$ \\
\hline 11 & 81 & 227 & 118 & 104 & 169 \\
\hline 22 & 83 & 198 & 80 & 100 & 161 \\
\hline 13 & 133 & 223 & 183 & 149 & $\angle 51$ \\
\hline 14 & 114 & 283 & $\angle 5 C$ & $\mathrm{CO} 3$ & $\angle 52$ \\
\hline 15 & 82 & 250 & 103 & 99 & 148 \\
\hline 16 & 87 & 219 & 29 & 92 & 134 \\
\hline 27 & 123 & 261 & $\angle 20$ & 158 & 144 \\
\hline 18 & 108 & 251 & $\angle 50$ & 137 & 144 \\
\hline 19 & 83 & 228 & 101 & 203 & 141 \\
\hline 20 & 84 & 200 & 82 & 100 & 132 \\
\hline 21 & $\angle 23$ & 223 & 153 & $N G$ & 145 \\
\hline 22 & 156 & 288 & 134 & $\therefore .1$ & 138 \\
\hline 23 & 134 & 136 & $\angle 31$ & $\therefore, \ldots$ & 128 \\
\hline 24 & 115 & 185 & 24 & 104 & 118 \\
\hline 25 & $|3|$ & 212 & 1021 & $\langle 5\rangle$ & $<47$ \\
\hline 26 & $\angle 13$ & 177 & 88 & $\angle 36$ & 145 \\
\hline 27. & 96 & $\angle 41$ & 81 & 102 & 134 \\
\hline 28 & 86 & 134 & 29 & $\angle 00$ & 124 \\
\hline 29 & 153 & 191 & 158 & $\angle 68$ & 150 \\
\hline 30 & 84 & $\angle 3\rangle$ & 148 & 135 & 140 \\
\hline 31 & 107 & $\angle 34$ & 88 & 98 & 123 \\
\hline 32 & 94 & 134 & 81 & 99 & 115 \\
\hline
\end{tabular}


241-SX SLUDGE PROFILE TEMPERATURES

(S.0.P. 200.3.6)

Time $\frac{i 030}{9-7-72}$

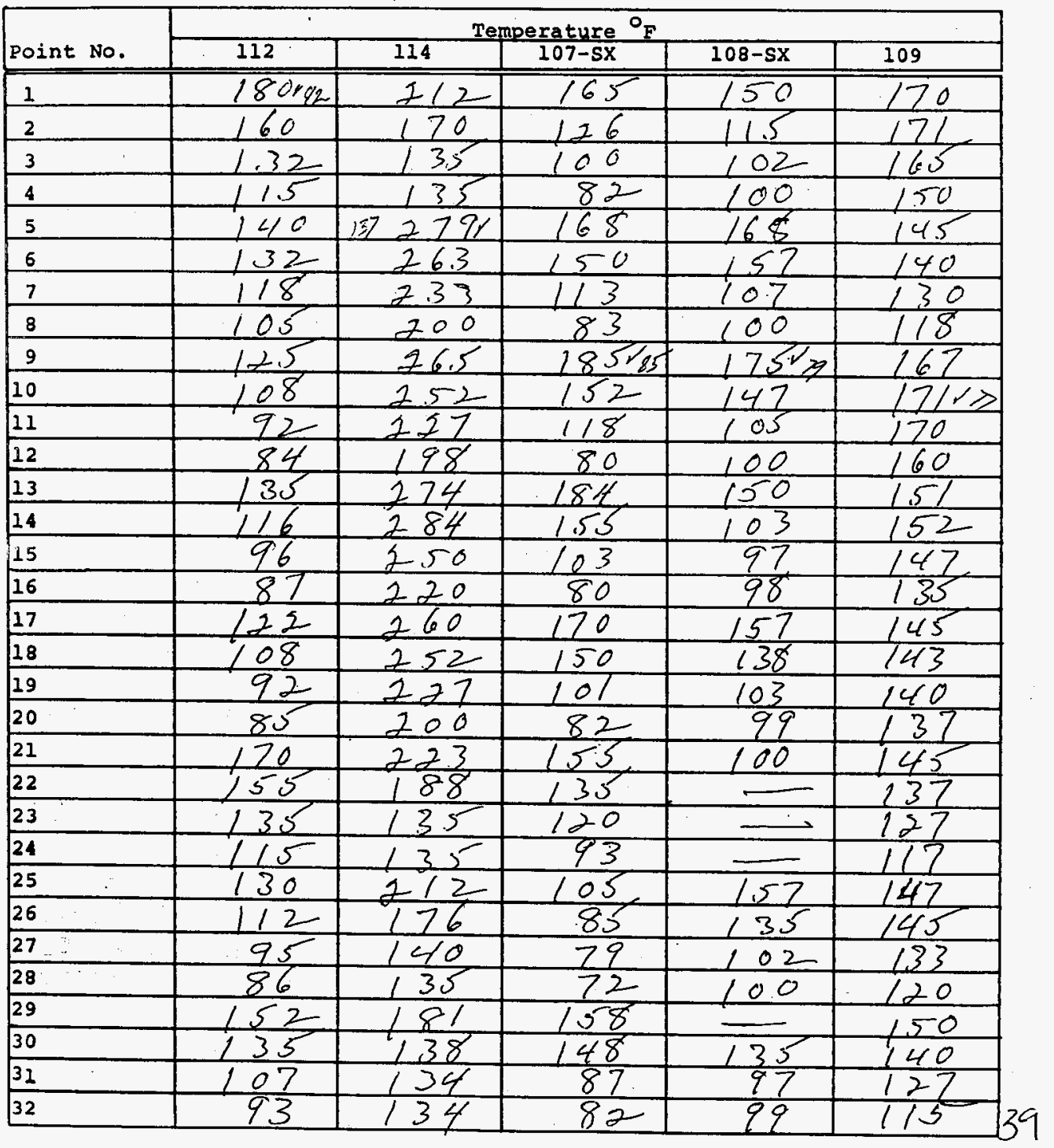


241-SX SLUDGE PROFILE TEMPERATURES

(S.0.P. 200.3.6)

Time $\frac{9 / 3 / 32}{0 / 40}$

\begin{tabular}{|c|c|c|c|c|c|}
\hline \multirow[b]{2}{*}{ Point No. } & \multicolumn{5}{|c|}{ Temperature ${ }^{\circ} F$} \\
\hline & 112 & 114 & $107-5 x$ & $108-5 x$ & 109 \\
\hline 1 & $180 \%$ & $2 / 3$ & $11:$ & $\angle 50$ & 169 \\
\hline 2 & 163 & 168 & , & 117 & 121 \\
\hline 3 & $|3\rangle$ & $\angle 34$ & 101 & 103 & 164 \\
\hline 4 & 115 & 134 & 83 & 101 & $\angle 50$ \\
\hline 5 & 141 & $298 \%$ & 168 & 168 & 145 \\
\hline 6 & 133 & 263 & 149 & 142 & 141 \\
\hline 7 & 118 & 233 & 114 & $10 s$ & 131 \\
\hline 8 & $\angle 03$ & 199 & 83 & 100 & 118 \\
\hline 9 & $\angle 25$ & 265 & 1858 & 126080 & 162 \\
\hline 10 & 10.8 & 251 & 162 & 148 & $1716 \geqslant$ \\
\hline 11 & 93 & 222 & 118 & 105 & 168 \\
\hline 12 & 83 & 199 & 80 & 160 & 160 \\
\hline 13 & $\angle 34$ & 273 & 183 & 149 & 151 \\
\hline 14 & 112 & 283 & 156 & 103 & 153 \\
\hline 15 & 97 & 250 & $\angle 03$ & 88 & 142 \\
\hline 16 & 87 & 219 & so & 99 & 134 \\
\hline 17 & $\angle 23$ & 261 & 130 & $\angle 58$ & 144 \\
\hline 18 & 108 & 251 & 150 & 132 & 144 \\
\hline 19 & 93 & 228 & $\angle 02$ & 163 & 141 \\
\hline 20 & 85 & 200 & 83 & 100 & 132 \\
\hline 21 & 174 & 274 & $\angle 56$ & $N C$ & 145 \\
\hline 22 & $\angle 52$ & 188 & $\angle 34$ & $x$ & 138 \\
\hline 23 & 135 & 135 & 122 & $x$ & 128 \\
\hline 24 & $1 / 4$ & 135 & 94 & 105 & 118 \\
\hline 25 & 131 & 212 & 105 & $\angle 52$ & 148 \\
\hline 26 & 113 & 122 & 88 & $13 c$ & 145 \\
\hline 27 & 94 & $\angle 41$ & 81 & 102 & 134 \\
\hline 28 & 87 & 134 & 29 & 100 & 119 \\
\hline 29 & $\angle 54$ & 191 & $\angle 59$ & $N G$ & 150 \\
\hline 30 & $\angle 35$ & 138 & 148 & 136 & 140 \\
\hline 31 & 108 & 134 & 88 & 98 & $12 ?$ \\
\hline 32 & 93 & 134 & 82 & 99 & 115 \\
\hline
\end{tabular}




\begin{tabular}{|c|c|c|c|c|c|}
\hline-511 & 007 & -28 & $H \varepsilon /$ & 76 & $z \varepsilon$ \\
\hline$\& \zeta$ & 66 & 88 & $t \in \varepsilon 7$ & 801 & $\tau \varepsilon$ \\
\hline 171 & उहा & $8+1$ & $0+1$ & $c \leqslant 1$ & $O E$ \\
\hline $0-51$ & द91 & 651 & 667 & HE ! & 62 \\
\hline 611 & 001 & 98 & $7 \varepsilon /$ & 68 & 82 \\
\hline$\neq \varepsilon 1$ & ह9/ & 28 & $1+1$ & 36 & $\angle 2$ \\
\hline $3+1$ & $3 \varepsilon /$ & $B S$ & 641 & 897 & 92 \\
\hline $4 t$ & 651 & 407 & $-6 / 6$ & चा। & $s z$ \\
\hline $81 /$ & $\$$ & 36 & $t+\varepsilon /$ & $7 / 1$ & 2 \\
\hline 661 & $x$ & $\tau \tau l$ & 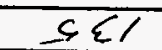 & $-4 \xi 7$ & $\varepsilon \tau$ \\
\hline $8 \varepsilon$ & $\bar{x}$ & दहा & 881 & 357 & 22 \\
\hline 9कर & $9 \pi$ & 657 & 7दर & Fl6 & $\tau z$ \\
\hline दहा & 00 & $\varepsilon \xi$ & 006 & trs & oz \\
\hline $1+1$ & $\varepsilon ण$ & c.01 & $\angle \epsilon 6$ & $\varepsilon 6$ & $6 \mathrm{~T}$ \\
\hline tht & 8हर & 05 & 156 & 807 & $8 \mathrm{~T}$ \\
\hline$t+1$ & 8.57 & 747 & 176 & म6द & $L \tau$ \\
\hline$F E-1$ & 007 & 08 & 616 & 68 & $9 \tau$ \\
\hline $8 \pi 1$ & 66 & -901 & 058 & 86 & ST \\
\hline$\varepsilon 5 !$ & 701 & 957 & $7+96$ & दा & $5 T$ \\
\hline-651 & bt/ & हना & A 46 & कह & $\varepsilon \tau$ \\
\hline 097 & 901 & 7.8 & 667 & $\varepsilon S$ & $2 \pi$ \\
\hline 897 & -507 & 6द & 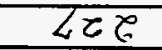 & $\sum 6$ & $\tau T$ \\
\hline $1161 \mathbb{L}$ & $8+1$ & $=97$ & 156 & 801 & $\overline{O T}$ \\
\hline 691 & $88 \times 347$ & 81587 & 576 & 567 & 6 \\
\hline 811 & 001 & $\xi \delta$ & 661 & +101 & 8 \\
\hline Iहा & 507 & 7r? & $\varepsilon \varepsilon E$ & 871 & $L$ \\
\hline$T \pi\rangle$ & दमt & 671 & f 76 & $\varepsilon \varepsilon /$ & 9 \\
\hline 351 & ह\% & 597 & 4866 & $7 A 7$ & $s$ \\
\hline 9-5! & 701 & 28 & Ћधा & 311 & $b$ \\
\hline 791 & मQ7 & 201 & महा & दहा & $\bar{\varepsilon}$ \\
\hline 161 & दा7 & दी & 397 & 797 & $\overline{2}$ \\
\hline 637 & कs? & 597 & दा 6 & 2087 & $\tau$ \\
\hline $60 \tau$ & $\mathrm{XS}-80 \tau$ & XS- $\angle O T$ & DIT & $2 \tau \tau$ & -ON 7UTTOd \\
\hline
\end{tabular}

\footnotetext{
ददयक

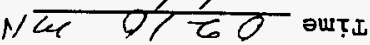


241-SX SLUDGE PROFILE TEMPERATURES

(5.0.P. 200.3.6)

Time $0120 \mathrm{mN}$. Date $9 / 5 / 72$

\begin{tabular}{|c|c|c|c|c|c|}
\hline \multirow[b]{2}{*}{ point No. } & \multicolumn{5}{|c|}{ Temperature ${ }^{\circ} F$} \\
\hline & 112 & 114 & $107-5 x$ & $108-5 X$ & 109 \\
\hline 1 & 182 & 212 & 165 & $\angle 50$ & 169 \\
\hline 2 & 166 & 168 & $\angle 22$ & $1 / 8$ & 126 \\
\hline 3 & 132 & 134 & 205 & $\angle O C$ & 164 \\
\hline 4 & 112 & 134 & 82 & 103 & 150 \\
\hline 5 & 142 & $228 \%$ & 165 & 168 & 145 \\
\hline 6 & 133 & $263^{\prime}$ & 249 & $\angle 488$ & 141 \\
\hline 7 & 118 & 233 & $\angle \angle 5$ & $110^{\circ}$ & 131 \\
\hline 8 & $\angle A 4$ & 199 & 88 & $\angle 02$ & 118 \\
\hline 9 & $\angle 25$ & 265 & $\angle 844^{8}$ & $126 \%$ & 167 \\
\hline 10 & 108 & 251 & 162 & 142 & $\Delta x_{6} 12$ \\
\hline 11 & 94 & 227 & $\angle 20$ & 102 & $\angle C 9$ \\
\hline 12 & 88 & 198 & 85 & 102 & 160 \\
\hline 13 & 134 & 274 & 183 & 149 & 152 \\
\hline 14 & 118 & 284 & $\angle 56$ & Lo? & 153 \\
\hline 15 & 99 & 250 & 108 & 100 & 147 \\
\hline 16 & 80 & 219 & 85 & 100 & 134 \\
\hline 17 & 124 & 261 & 170 & 158 & 144 \\
\hline 18 & 109 & 251 & 150 & 138 & 143 \\
\hline 19 & 92 & 227 & $\angle 05$ & $\angle 05$ & 141 \\
\hline 20 & 88 & 199 & 88 & 101 & 137 \\
\hline 21 & 124 & 223 & $\angle 56$ & $N G$ & 145 \\
\hline 22 & 157 & 188 & 134 & 11 & $\angle 38$ \\
\hline 23 & $\angle 34$ & 135 & $\angle 23$ & 11 & $\angle 28$ \\
\hline 24 & $\angle 15$ & 134 & 29 & 1. & 118 \\
\hline 25 & 132 & $2 / 2$ & 102 & 152 & $\angle 47$ \\
\hline 26 & 114 & $17 ?$ & 92 & 132 & 145 \\
\hline 27. & 92 & 140 & 82 & $\angle 04$ & 134 \\
\hline 28 & 90 & 134 & 85 & 101 & 119 \\
\hline 29 & $\angle 54$ & 192 & 158 & $\angle 67$ & 150 \\
\hline 30 & 135 & 138 & $\angle 468$ & $\angle 3 C$ & 140 \\
\hline 31 & 110 & $13+6$ & 93 & 100 & 127 \\
\hline 32 & 97 & 134 & 87 & 100 & 115 \\
\hline
\end{tabular}


241-SX SLUDGE PROFILE TEMPERATURES

$(S .0 . P .200 .3 .6)$

Time $\frac{0 / 40}{9 / 4 / 22 \geqslant N}$

\begin{tabular}{|c|c|c|c|c|c|}
\hline \multirow[b]{2}{*}{ Point No. } & \multicolumn{5}{|c|}{ Temperature ${ }^{\circ} F$} \\
\hline & 112 & 114 & $107-5 x$ & $108-5 x$ & 109 \\
\hline 1 & 180 & $2 / 2$ & 165 & $\angle 50$ & 169 \\
\hline 2 & 163 & 162 & 127 & $\angle \angle 8$ & 171 \\
\hline 3 & 137 & 134 & 104 & 105 & 164 \\
\hline 4 & 116 & 134 & 85 & $\angle 03$ & $\angle 50$ \\
\hline 5 & 141 & 228 & 168 & 168 & 145 \\
\hline 6 & 133 & 264 & 149 & 148 & 241 \\
\hline 7 & 118 & 233 & 115 & 109 & 131 \\
\hline 8 & 104 & 199 & 57 & $\angle 01$ & 118 \\
\hline 9 & $\angle 25$ & 264 & 184 & 126 & 162 \\
\hline 10 & 108 & 251 & 162 & 148 & 171 \\
\hline 11 & 94 & 227 & $\angle 19$ & 102 & 169 \\
\hline 12 & 86 & 199 & 83 & $\angle 01$ & 160 \\
\hline 13 & 135 & 224 & 183 & $\angle 48$ & 151 \\
\hline 14 & 117 & 284 & $\angle 56$ & 105 & $\angle 52$ \\
\hline 15 & 88 & 250 & $\langle 0\rangle$ & 100 & 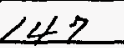 \\
\hline 16 & 89 & 219 & 83 & $\angle 00$ & 134 \\
\hline 17 & $\angle 24$ & 261 & 120 & $\angle 58$ & $\angle 44$ \\
\hline 18 & 109 & 250 & $\angle 50$ & $\angle 38$ & 143 \\
\hline 19 & & $22 ?$ & 104 & $\angle 05$ & 141 \\
\hline 20 & 88 & 199 & 86 & $\angle 01$ & 136 \\
\hline 21 & 124 & 223 & $\angle S C$ & $1 / G$ & 145 \\
\hline 22 & 156 & 188 & $<34$ & 2 & $\angle 38$ \\
\hline 23 & $\angle 35$ & 135 & $\angle 22$ & & 128 \\
\hline 24 & $\angle \angle 5$ & $\angle 35$ & 22 & 167 & $\angle 18$ \\
\hline 25 & $\angle 31$ & 212 & 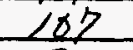 & $\angle 52$ & 142 \\
\hline 26 & 114 & 192 & 91 & 137 & 145 \\
\hline 27 & 92 & 142 & 85 & 103 & $\angle 34$ \\
\hline 28 & 88 & $\angle 34$ & 83 & 100 & $\angle 19$ \\
\hline 29 & 255 & 192 & $\angle 58$ & 168 & $\angle 50$ \\
\hline 30 & $\angle 36$ & $\angle 40$ & $\angle 4 \angle 8$ & 136 & 140 \\
\hline 31 & $\angle 09$ & 133 & 82 & $\angle 00$ & $\langle 2\rangle$ \\
\hline 32 & 86 & 133 & 84 & $\angle 00$ & 115 \\
\hline
\end{tabular}


241-SX SLUDGE PROFILE TEMPERATURES

(S.0.P. 200.3.6)

Time $\frac{\angle 300}{8-31-72}$

\begin{tabular}{|c|c|c|c|c|c|}
\hline \multirow[b]{2}{*}{ point No. } & \multicolumn{5}{|c|}{ Temperature ${ }^{\circ} F$} \\
\hline & 212 & 114 & $107-5 x$ & $108-5 x$ & 109 \\
\hline 1 & 150 & 212 & 16 & 150 & 120 \\
\hline 2 & 162 & 165 & 126 & $118^{2}$ & 170 \\
\hline 3 & 137 & 135 & 104 & 105 & 6 \\
\hline 4 & 118 & 135 & 25 & 100 & 150 \\
\hline 5 & 140 & 122 & 165 & 163 & 145 \\
\hline 6 & 132 & 80 & 150 & 145 & 140 \\
\hline 7 & 118 & 265 & 115 & 110 & 130 \\
\hline 8 & 205 & 235 & 86 & 100 & 120 \\
\hline 9 & 175 & 200 & 18 & 125 & 165 \\
\hline 10 & 110 & 240 & 760 & 145 & 170 \\
\hline 11 & 90 & $25-0$ & 120 & 105 & 120 \\
\hline 12 & -85 & 225 & 85 & 100 & 160 \\
\hline 13 & 135 & 200 & 182 & 150 & 250 \\
\hline 14 & 115 & 275 & 155 & 105 & 250 \\
\hline 15 & 100 & 280 & 105 & 100 & 145 \\
\hline 16 & 90 & 250 & 85 & 100 & 13 \\
\hline 17 & 125 & 220 & 120 & 155 & 145 \\
\hline 18 & 110 & 260 & 150 & 135 & 145 \\
\hline 19 & 95 & 250 & 100 & 105 & 140 \\
\hline 20 & 90 & $2 \leqslant 5$ & 85 & 100 & 135 \\
\hline 21 & 1.70 & 200 & 155 & 120 & 145 \\
\hline 22 & 155 & 225 & 135 & 135 & 135 \\
\hline 23 & 835 & 1.85 & 120 & 115 & \\
\hline 24 & 113 & 140 & 70 & 105 & 11 \\
\hline 25 & 115 & 140 & 105 & 155 & 145 \\
\hline 26 & 95 & 212 & .85 & 135 & 145 \\
\hline 27 & 90 & $22^{2} 6$ & 83 & 100 & 133 \\
\hline 28 & 152 & 140 & 83 & 100 & 120 \\
\hline 29 & 13.5 & 135 & $\angle 58$ & 180 & 150 \\
\hline 30 & 110 & 190 & +18 & 135 & 140 \\
\hline 31 & 95 & 140 & 92 & 100 & 125 \\
\hline 32 & 88 & 13.5 & 85 & 100 & $1 / 5$ \\
\hline
\end{tabular}


241-SX SLUDGE PROFILE TEMPERATURES

$(S .0 . P .200,3,6)$

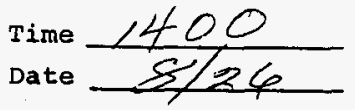

\begin{tabular}{|c|c|c|c|c|c|}
\hline \multirow[b]{2}{*}{ Point No. } & \multicolumn{5}{|c|}{ Temperature ${ }^{\circ} F$} \\
\hline & 112 & 114 & $107-5 x$ & $208-5 x$ & 109 \\
\hline 1 & 150 & $2 / 2$ & $16<$ & $\angle 50$ & $16=8$ \\
\hline 2 & 163 & 166 & 126 & 112 & 171 \\
\hline 3 & $\angle 35$ & 133 & 164 & 105 & 163 \\
\hline 4 & 115 & 133 & $\& 8$ & 103 & 150 \\
\hline 5 & 140 & 278 & 168 & $\angle 63$ & 144 \\
\hline 6 & 132 & $\Rightarrow<<x$ & 148 & 142 & 141 \\
\hline 7 & 113 & 232 & 114 & 110 & 131 \\
\hline 8 & 100 & 198 & 89 & 102 & 118 \\
\hline 9 & 125 & 263 & 184 & 126 & 162 \\
\hline 10 & 108 & 250 & $\angle L 1$ & 147 & 122 \\
\hline 11 & 94 & 224 & 117 & 107 & 169 \\
\hline 12 & 90 & 194 & 88 & $\angle 03$ & 160 \\
\hline 13 & $\angle 3 \angle$ & 274 & 183 & 149 & 151 \\
\hline 14 & 119 & 285 & 155 & $20 ?$ & 151 \\
\hline 15 & 98 & 248 & 102 & $100^{\circ}$ & 146 \\
\hline 16 & 92 & 218 & 88 & 100 & 134 \\
\hline 17 & $\angle 23$ & 258 & 120 & 152 & 144 \\
\hline 18 & 168 & 250 & 150 & 137 & 143 \\
\hline 19 & 95 & 225 & $205^{\circ}$ & 183 & $\angle 40$ \\
\hline 20 & 90 & 195 & 88 & 100 & 136 \\
\hline 21 & 124 & 222 & 155 & $1 / 6$ & 145 \\
\hline 22 & 135 & 284 & $\angle 33$ & 1.1 & 132 \\
\hline 23 & 133 & 133 & 122 &. .1 & 122 \\
\hline 24 & 114 & 133 & 88 & $\because \quad 1$ & 112 \\
\hline 25 & 130 & 212 & $105^{\circ}$ & $\angle 52$ & 142 \\
\hline 26 & 112 & 126 & 90 & 136 & 145 \\
\hline 27 & 95 & 141 & 29 & 103 & 133 \\
\hline 28 & 90 & $134 \neq 0$ & 28 & 201 & 118 \\
\hline 29 & 153 & 190 & 152 & $\angle 62$ & 150 \\
\hline 30 & $\angle 35$ & 140 & 142 & 135 & $\angle 40$ \\
\hline 31 & $\angle 10$ & 133 & 93 & 100 & $1 \leq c$ \\
\hline 32 & 94 & 133 & 82 & 101 & 114 \\
\hline
\end{tabular}


241-SX SLUDGE PROFILE TEMPERATURES

$(S .0 . P .200 .3 .6)$

Time $\frac{1345}{8-24-72}$

\begin{tabular}{|c|c|c|c|c|c|}
\hline \multirow[b]{2}{*}{ Point No. } & \multicolumn{5}{|c|}{ Temperature ${ }^{\circ} \mathrm{F}$} \\
\hline & 112 & 114 & $107-5 x$ & $108-5 x$ & 109 \\
\hline 1 & 181 & $9 / 1$ & 164 & 150 & 169 \\
\hline 2 & 165 & 16.3 & 1.26 & 117 & 171 \\
\hline 3 & 1.36 & 133 & 103 & 104 & 164 \\
\hline 4 & 115 & 1.34 & 86 & 102 & 1.50 \\
\hline 5 & 140 & 279 & 168 & 167 & 144 \\
\hline 6 & 132 & 264 & 148 & 146 & 135 \\
\hline 7 & 117 & 232 & 114 & 109 & 130 \\
\hline 8 & 104 & 197 & 87 & 101 & 118 \\
\hline 9 & 125 & 263 & 184 & 175 & 167 \\
\hline 10 & 108 & -250 & 161 & 145 & 170 \\
\hline 11 & 93 & 253 & 118 & 106 & 168 \\
\hline 12 & 87 & 194 & 85 & 102 & 160 \\
\hline 13 & 134 & 274 & 183 & 148 & 151 \\
\hline 14 & 116 & 286 & 155 & 105 & 152 \\
\hline 15 & 98 & 248 & 106 & 99 & 146 \\
\hline 16 & 90 & 218 & 85 & 99 & 133 \\
\hline 37 & 122 & 258 & 169 & 157 & 144 \\
\hline 18 & 108 & 250 & 150 & 136 & 143 \\
\hline 19 & 94 & 224 & 104 & 102 & 140 \\
\hline 20 & 89 & 194 & -86 & 100 & 135 \\
\hline 21 & 173 & 221 & 155 & - & 1465 \\
\hline 22 & 150 & 183 & 134 & 5 & 137 \\
\hline 23 & 1.33 & 134 & 150 & $=$ & 127 \\
\hline 24 & 114 & 134 & 95 & $=$ & $1 / 6$ \\
\hline 25 & 130 & 212 & 105 & 156 & 146 \\
\hline 26 & 112 & 176 & 88 & 135 & 144 \\
\hline 27 & 94 & 141 & 82 & 102 & 633 \\
\hline 28 & 89 & 134 & 81 & 100 & 118 \\
\hline 29 & 154 & 190 & 157 & 167 & 149 \\
\hline 30 & 135 & 142 & 146 & 135 & 140 \\
\hline 31 & 108 & 133 & 92 & 99 & 126 \\
\hline 32 & 95 & 134 & 86 & 102 & 108 \\
\hline
\end{tabular}


WHC-SD-LM-DP-232, ReV. O

241-SX SLUDGL PROFIIE TENPERAMURES

(S.0.P. 200.3.6)

Time $\frac{01 / 5}{4-5-72}$

Point No.

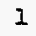

2

3

4

5

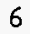

7

8

9

10

11

12

13

14

15

16

17

18

19

20

21

22

23

24

25

26

27

28

29

30

31

32
Temperature ${ }^{\circ}$ F $1075 x=170$

$107-5 x$

230

193

$\frac{193}{119}$

291

281

255

225

288

278

255

225

281

290

260

232

286

281

260

230

216

$\frac{216}{158}$

$\frac{158}{120}$

119

220

183

143

$\frac{119}{192}$

$\frac{192}{129}$

120

119

$\underline{108-5 x}=165$

$1095 x=170$

$1125 x=160$
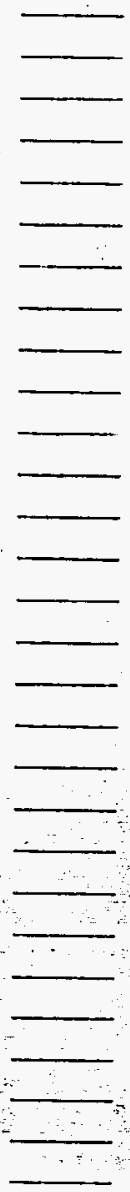
241-SX SLUDGE PPOFILE TDTPERATUTES (S.0.P. 200.3.6)

Point jo.

1

2

3

4

5

6

7

8

9

10

11

12

13

14

15

16

17

18

19

20

21

22

23

24

25

26

27

28

29

30

31

32
165

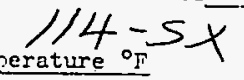

Time

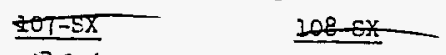

224

193

143

118

292

283

251

225

288

278

256

226

282

290

$2<?$

233

287

282

$2<2$

231

317

152

$118:$

118

221

182

142

117

193

132

118

118
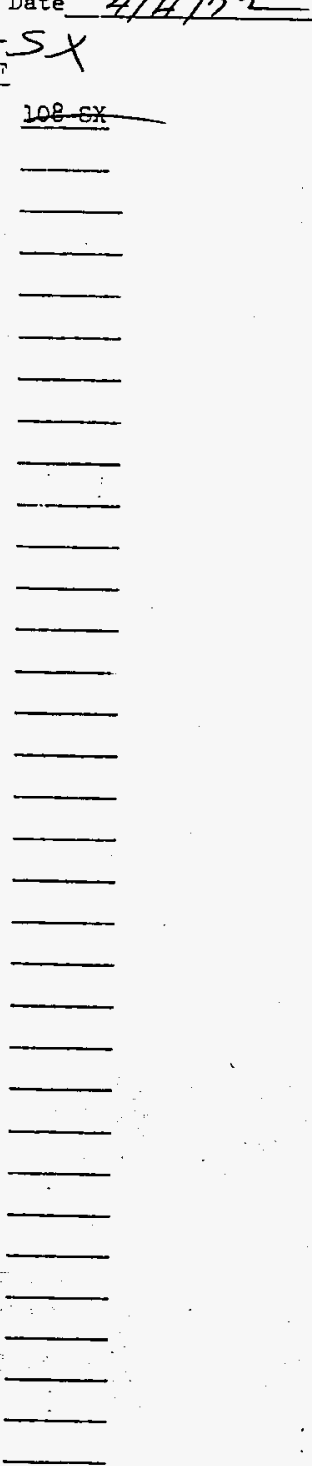
(S.0.P. 200.3.6)

Point No.

1000

ra

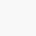

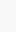

(n)

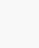

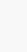

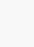

1

13

4

6

8

0

2

3

4.

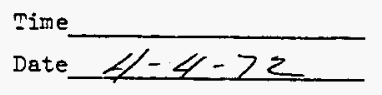


(S.0.P. 200.3.6)

Point Io.

1

2

3

4

5

6

7

8

9

10

11

12

13

14

15

16

17

18

19

20

21

22

23

24

25

26

27
-28

28

$\because 29$

30

31

32

\section{.}

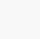

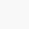

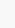

6

(

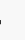

(1)

2

3

4

6

7

18

9 .

0

. 
(S.0.P. 200.3.6)

mime $\frac{0100}{4-3-72}$

Point No.

\section{1}

2

3

4

5

6

7

8

9

10

11

12

13

14

15

16

17

18

19

20

21

22

23

24

25

26

27

28

29

30

31

32
$1 / 4$ Temperature ${ }^{\circ} \mathrm{F}$

Leas 5

230

$\frac{230}{191}$

145

120

290

282

260

225

287

276

255

227

280

290

261

234

285

281

262

$\frac{232}{217}$

160

$\frac{162}{122}$

122

220

$\frac{220}{183}$.

146

121

$\frac{121}{1.95}$

124

$\frac{122}{120}$
HI TeMyps $\underline{108-5 x}=165^{\circ}$

$107=170^{\circ}$

$109=166^{\circ}$

$112=159^{\circ}$
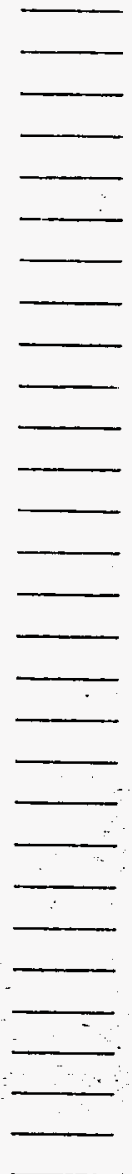


\section{(S.0.P. 200.3.6)}

Point llo.

1

2

3

4

5

6

7

8

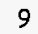

10

11

12

13

14

15

16

17

18

19

20

21

22

23

$: 24$

25

26

27

28

29

30

31

32
$1145 x$

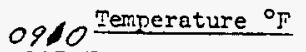

$+2$

229

192

185

122

251

28

257

225

782

226

205

226

$\frac{280}{280}$

289

$26 /$

213

285

280

26

231

216

157

123

122

221

182

$14 / 15 \div$

$12 \%$

195

124

122

122

Time

Date $4-3-72$

1570

230

192

145

120

$24 \angle$

282

254

225

$2 \times 7$

217

255

225

281

289

160

232

285

280

260

231

216

157

120

120

$22 \%$

183

145

120

195

121

120

120
1700

2300

230230

192192.

144 .145

120,30

292292.

$2 \& 3252$

$258=27$

$226 \quad 226$

$255 \quad 25\}$

277208 .

$256 \quad 256$

226226 .

$282=81$.

292296.

$262 \quad 262$.

233233 .

$287=86$.

से 282.

$263=62$.

$232+32$

$2,7 \quad 217$

157 157

$119 \quad, \geqslant 1$

$119 \quad 221$

121.222

183,83

145,146

$1 / 9<20$.

$194 / 93$

126,21

$119+21$

$119 / 1 \%$. 
241-SX SIUDGE PROFILE TEMPERATURES

$\frac{(\mathrm{S} .0 . \mathrm{P} \cdot 200 \cdot 3.6)}{(4-5)}$

Dime $\frac{0200}{4-2-72}$

Point Ho.

1

2

3

4

5

6

7

8

9

10

11

12

13

14

15

16

17

18

19

20

21

$=\infty$

$22 \div 2 \%$

23

24

25

26

27

28

29.

30

101

31.

32

$1 / 4$ Temperature ${ }^{\circ} \mathrm{p}$

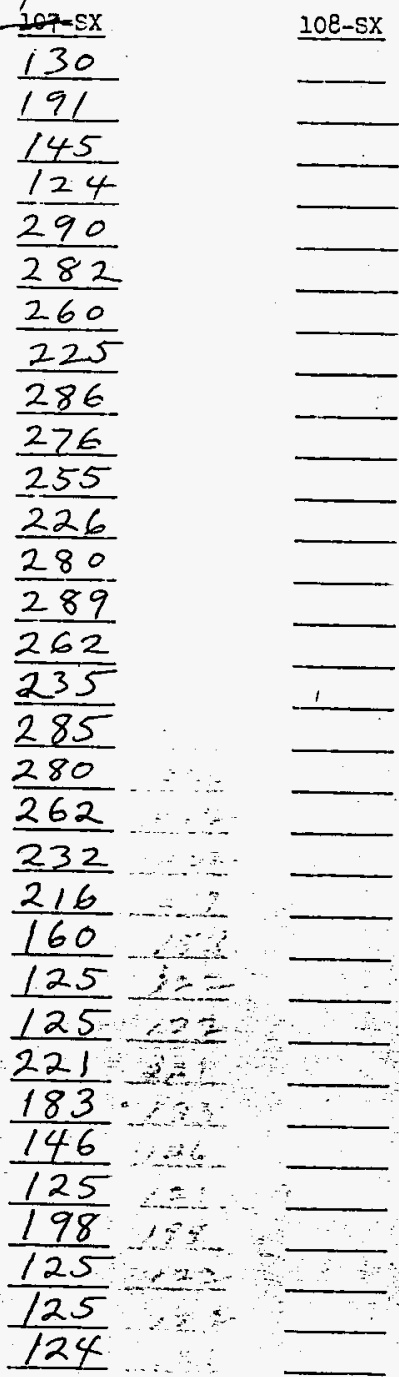

$+$

1

Date $4-2-72$

108-SX

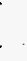


241-EX SLUDGE PPOFILE TDUPERATURES

Point Ho.

$$
1
$$

2

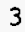

4

5

6

7

8

20

11

12

13

14

15

16

17

18

19

20

21

22

23

24

25

26

27.

28

29.

30

32

32
(S.0.P. 200.3.6)

0900

Time

Date $4-2-72$

$1 / 4$ Temperature of 1430

192

230

146

$-125$

290

282

257

226

286

277

$\frac{255}{25}$

226

280

289

262

234

285

280

192

145

122

290

282

$\frac{282}{257}$

225

286

277

$\frac{272}{255}$

$\$ 26$

580

289

261

235

285

280

262

262

232

232

216

160

$\frac{160}{125}$

125

221

182 .

147

125

198

127

$\frac{125}{125}$

122

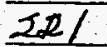

182

147

122

198

II3

122

122 
241-SX. SLUDGE PROFILE TPIPERATURES

(5.0.P. 200.3.6)

Point No.

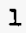

2

3

4

5

6

7

8

9

10

11

12

13

14

15

16

17

18

19

20

21

22

23

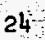

25

26

27

28

29

30

31 32
1030

1145 Anmerature ${ }^{\circ} \mathrm{F} / 445^{-}$ stx

229

191

145

126

290

282

2.58

226

286

272

255

236

280

288

262

235

285

279

262

$\frac{132}{216}$

160

126

126

220 .

$\frac{182}{142}$

$145 / 47$

126

260

$\frac{\frac{126}{126}}{126}$

224

191

145

125

290

282

258

226

286

277

255

226

280

$\frac{280}{288}$

262

235

285

$2>9$

261

232

$2 / 6$

160

125

125

210

182

$\frac{182}{147}$

124

200

125

$\frac{125}{124}$

124
Time

Date

$4-1->2$ 
Point No.

26

27

28

29

30

31 32
(1) $114-5 x$

Time

Date $4 / 1 / 22$

$207 \mathrm{sx}$

229

192

145

124

290.

282

258

227

287

227

$-255$

227

279

288

262

234

285

229

$2<2$

232

$\frac{212}{159}$

124

124

221

183

144

124

200

125

124

124
2300

321

$\angle 92$

145

124

290

282

258

221

$-287$

$278-10$

$\frac{256}{27}-11$

$22 ?$

$280-13$

288

262

$235-16$

255

$280<18$

$263<19$

232

2,1

159

125

125

222

$\ddot{83}$

147

124

199

$1=5$

124

$1 \geq 4$ 
Point No.

1

2

3

4

5

6

7

8

9

10

11

12

13

14

15

16

17

18

19

20

21

22

23

24

25

26

27

28

29

30

31

(1)

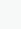

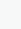

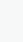

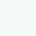

(1)

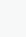

10

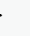

3

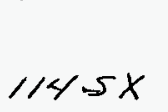

Time

Date $3-3 /-20$

Time 0945 Temperature ${ }^{\circ} \mathrm{F} @ 1700$ 
241-GX SLUDGE PROFILE TEMPERAFURES

(S.0.P. 200.3.6)

Time $0 / 0 Q$

Point No.

$1 / 4 /$ Temperature ${ }^{\circ} \mathrm{F}$

1

2

3

4

5

6

7

8

9

10

11

12

23

14

15

16

17

18

19

20

21

22

23

24

25

26

27

28

29

30

31

32
$108-5 x$

230

$\frac{190}{145}$

290

286

260

230

286

278

257

225

280.

290

265

235

280

280

265

335

217

160

$\angle 30$

130

223

182

145

127

205

$\angle 30$

$\angle 29$

$\angle 29$ 
241-SX SLUIDCE PROFILE TEMPERATURES

TIME 08.45 DATE 2.24 .72

BEST AVALABLE COPY

\section{(1) T.C.} \begin{tabular}{ll|l|l|l|l|l|l|l} 
& & & \\
T.C. Probe & 21 & 29 & 38 & 46 & 54 & PTs. \#
\end{tabular} $\mathrm{mx}-207$

TK -108

$\overline{2} x-109$

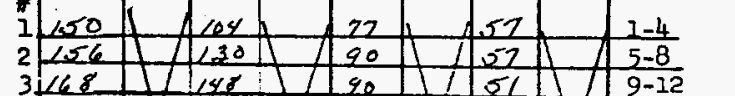
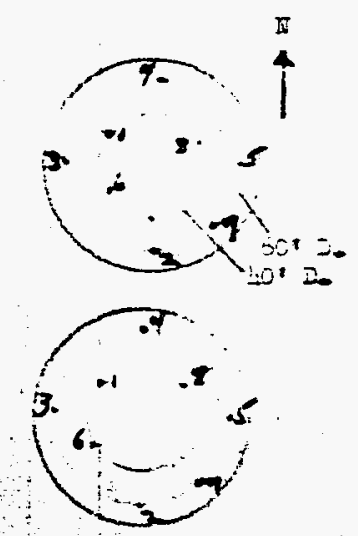

$x+x+4$

\begin{tabular}{|c|c|c|c|c|c|}
\hline 167 & 134 & 29 & $y$ & 54 & $13-16$ \\
\hline 157 & 126 & 25 & 7 & 55 & $17-20$ \\
\hline 143 & 116 & 96 & I & 69 & $21-24$ \\
\hline 80 & 60 & 58 & 1 & 51 & $25=28$ \\
\hline 140 & $\angle 26$ & C6L & & 54 & $29-32$ \\
\hline
\end{tabular}

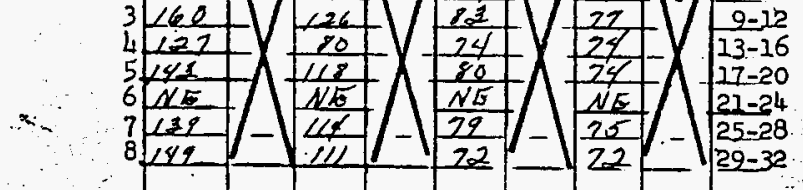

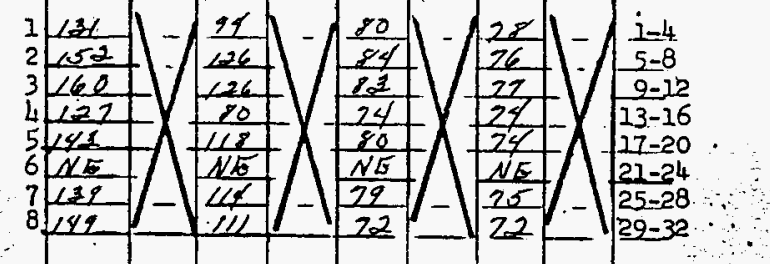
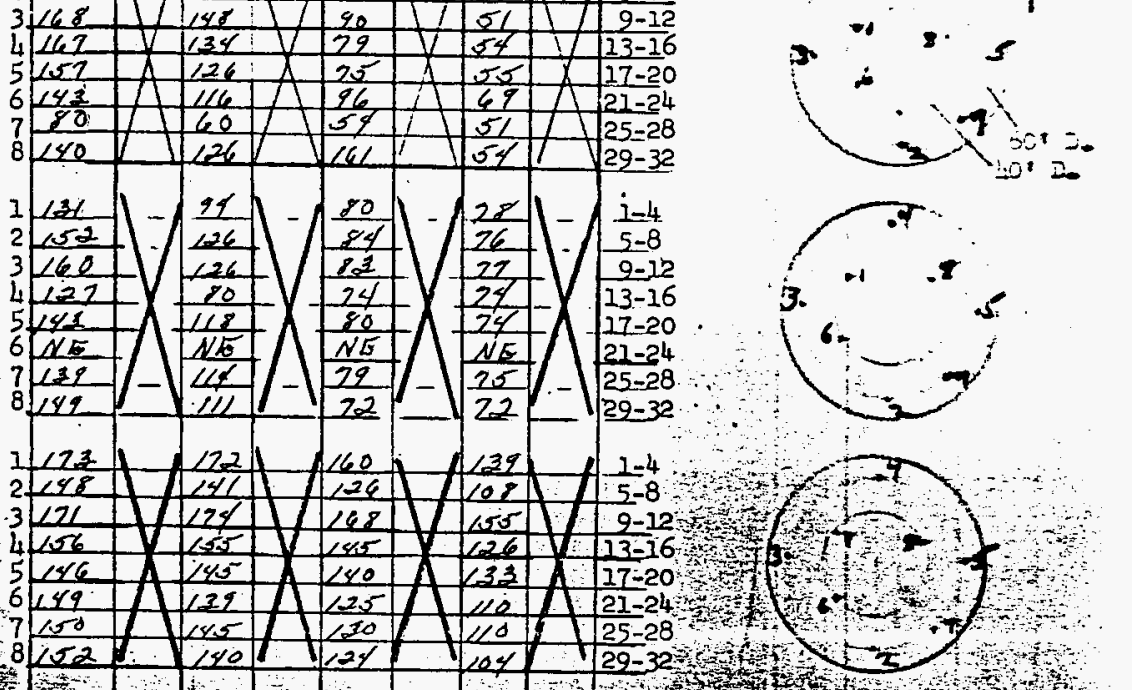

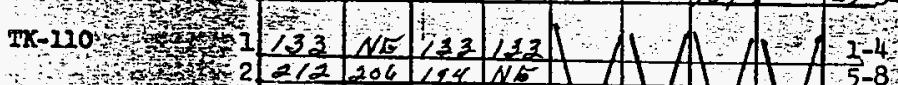

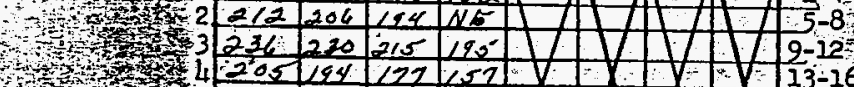

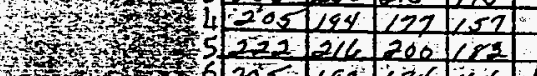

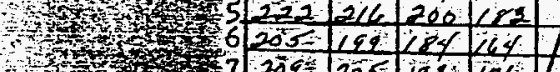

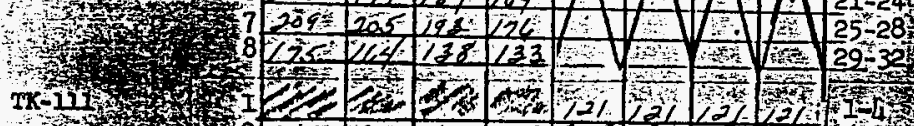

$A A A C A \begin{aligned} & 13-16 \\ & 17-20 \\ & 21-24\end{aligned}$

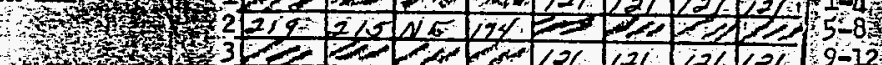

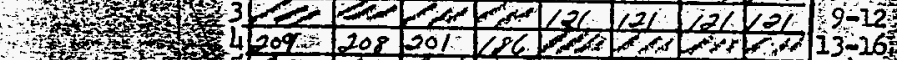

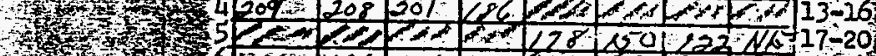

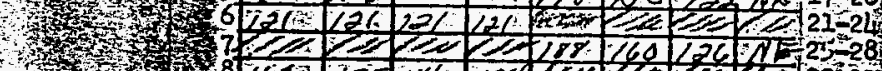

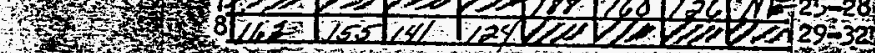

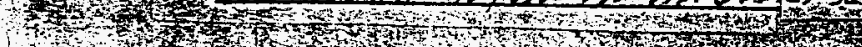

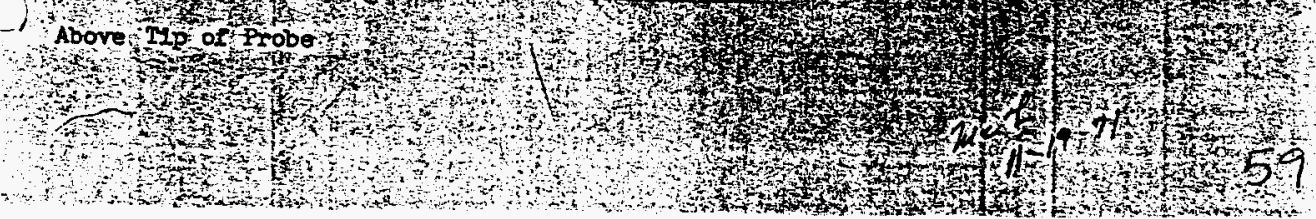


BEST AVAiLABLE COPY
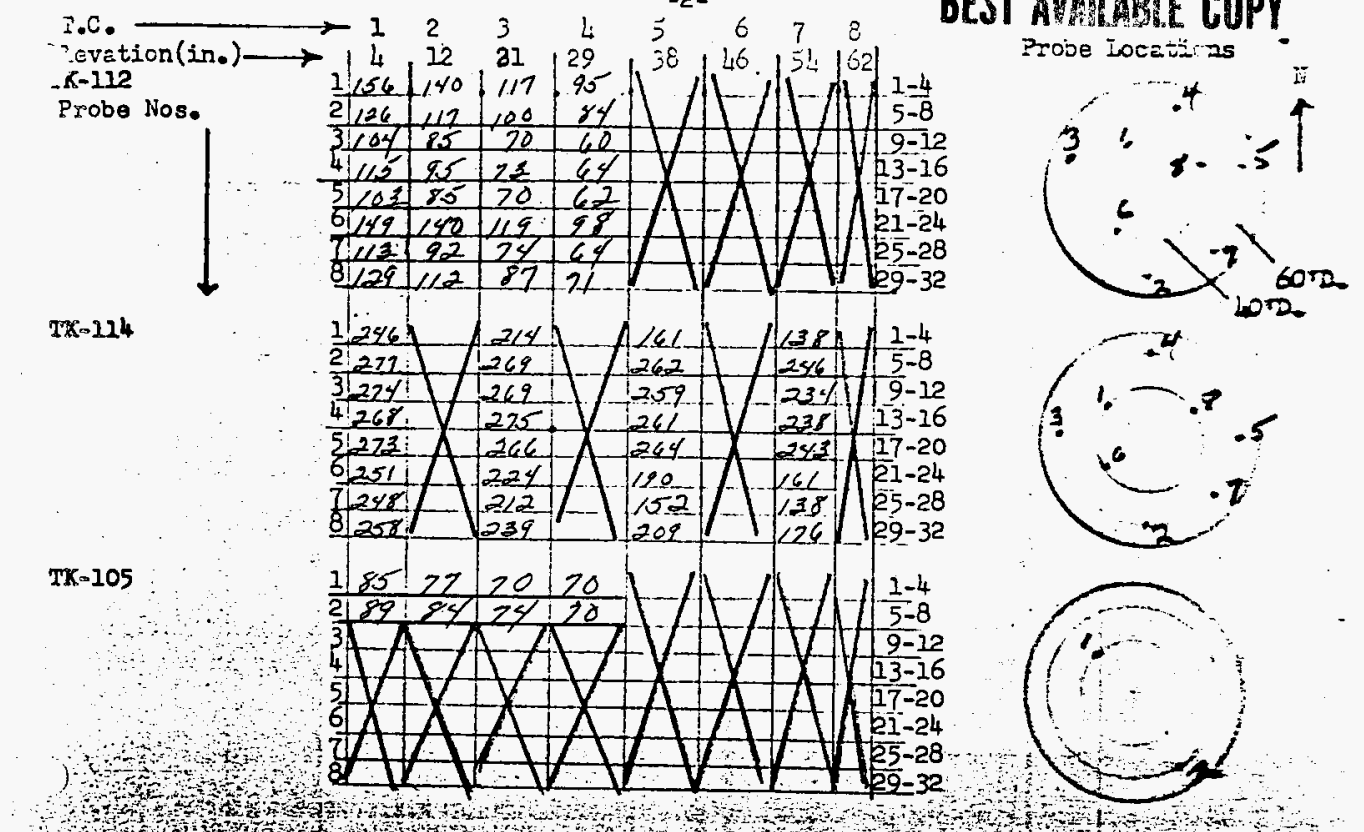
241-SX SLUDGI PROFILE TRMPERATURIS

(S.0.P. 200.3.6)

Point No.

1

2

3

4

5

6

7

8

9

10

11

12

13

14

15

16

17

18

19

20

21

22

23

24

25.

26

27

28.

29

30

31

32
$1 / 4 \rightarrow$ Temperature ${ }^{\circ} \mathrm{F}$ 
241-SX SIUDGE PROFILE TTMPERATURES (5.0.P. 200.3.6)

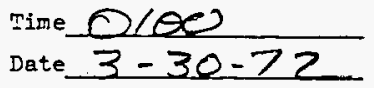

Point Ho.

1

2

3

4

5

6

7

8

9

10

11

12

13

14

15

16

17

18

19

20

21

22

23

24

स25

. 6

27

27

29

$\leq 29$

23030

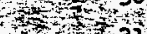

32
$1 / 4$ Temperature ${ }^{\circ} \mathrm{F}$

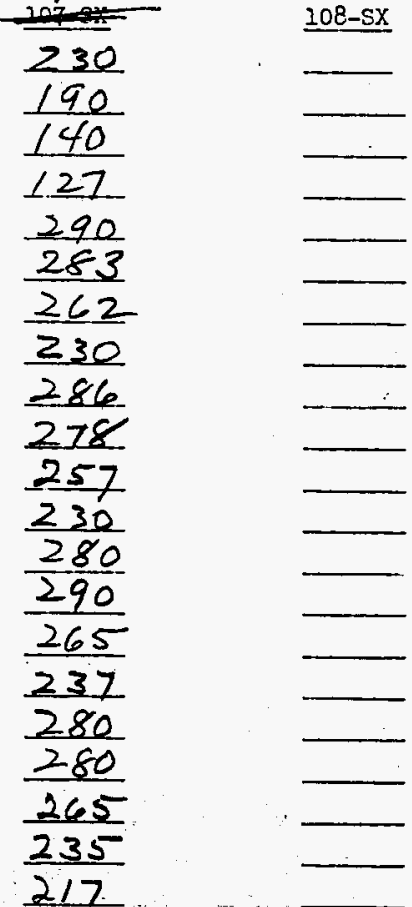

160

130

130

223

180.

145

227

207

135

130

129 


Time $\frac{1630-1700}{3 / 29 / 92}$

Point Ho.

22

23

24

25

26

27

28

29

30

31.

32

Temperature ${ }^{\circ} \mathrm{F}$

\section{7-SX}

151

108

$-85$

$-68$

151

133

96

$\frac{69}{120}$

143

$-\frac{58}{65}$

120

143

$\frac{87}{67}$

42

154

$\angle 34$

85

68

144

120

100

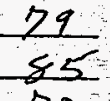

70

64

63

143

130

22

67
$108-5 x$

135

100

88

87

156

$\frac{132}{93} \frac{86}{124} \frac{131}{91}$

56

., 33

90

$-85$

84

149

$\frac{124}{90}$

85

$\sqrt{6}$

$x$
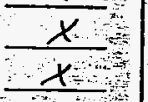

143
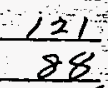

$8=85$

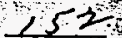

118

$-83$

83

\begin{tabular}{|c|c|c|}
\hline 09 & 112 & 4 \\
\hline 171 & 158 & 229 \\
\hline 170 & 143 & 188 \\
\hline 154 & 118 & 140 \\
\hline 140 & 94 & 254 \\
\hline 144 & 128 & 281 \\
\hline 139 & 118 & $2<0$ \\
\hline 125 & 102 & 237 \\
\hline 108 & 88 & 284 \\
\hline $1<8$ & 108 & $23 ?$ \\
\hline $1 \rightarrow 2$ & 90 & 256 \\
\hline $1<7$ & 77 & 227 \\
\hline .55 & 70 & 279 \\
\hline 153 & 117 & 258 \\
\hline 152 & 98 & 263 \\
\hline 148 & 19 & 234 \\
\hline 127 & 13. & 284 \\
\hline 143 & 106 & 238 \\
\hline 142 & 89 & $2<3$ \\
\hline 138 & .77 & 233 \\
\hline 132 & 31 & 2,3 \\
\hline 44 & 150 & 158 \\
\hline 132 & 141 & 128 \\
\hline 124 & 119 & 128 \\
\hline 1008 & 99 & 222 \\
\hline 149 & 114 & 780 \\
\hline 144 & 95 & 143 \\
\hline 129 & 79 & 128 \\
\hline 11 & 173 & 208 \\
\hline 150 & 133 & 134 \\
\hline 137 & $=125$ & 18 \\
\hline 120 & 90 & 125 \\
\hline$<0 z$ & 28 & \\
\hline
\end{tabular}


WHC-SO-WMH-DP-232, Rev. O

SX SLUDGL PROFILE TDMPERATURES

$$
\begin{aligned}
& \text { (S.0.P. 200.3 } \\
& \text { 'A' 4-12 }
\end{aligned}
$$

Point No.

1

2

3

4

5

6

7

8

9

10.

11

12

13

14

15

16

17

18

19

20

21

22

23

24

25

26

27.

28.

29

30

31
Time $\frac{1720}{3 / 24 / 72}$

C. $12-8$

114 Temperature ${ }^{\circ} \mathrm{C} / 14$

$3-27-72$
IOT-SX

225

$\frac{185}{135}$

$\frac{-\frac{125}{290}}{\frac{282}{265}}$

$\frac{\frac{108-5 x}{225}}{\frac{185}{135}}$

$\frac{\frac{126}{290}}{\frac{183}{263}}$

230

287

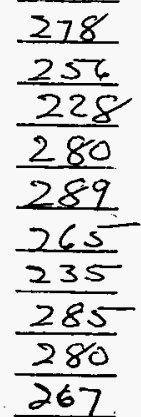

$\frac{\frac{236}{220}}{\frac{155}{\frac{128}{128}}} \frac{224}{\frac{180}{140}}$

$\frac{\frac{127}{221}}{\frac{150}{127}} \cdot \frac{\frac{127}{220}}{\frac{149}{125}} \cdot \frac{127}{125}$

64 
Point Ho.

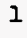

2

3

4

5

6

7

8

9

10

11

12

13

14

15

16

17

18

19

20

21

22

23

24

25

26

27

28

29

30

31

32
$1 / 4$ Temperature ${ }^{\circ} \mathrm{F}$

$167-5 \mathrm{x}$ 108-5X

227

182

130

125

289

281

261

230

$\frac{285}{285}$

276

2.55

226

277

287

260

234

283

278

265

235

221

150

126

126

$\frac{126}{221}$

176.

$\frac{176}{134}$

126

$\frac{126}{225}$

161

126

126 
242-S\% SLUDGI PROFILE TTMPERARURES

(5.0.P. 200.3.6)

$$
A-4-12
$$

Dime $\frac{1705}{3-24-72}$

Point No.

114 Temperature ${ }^{\circ} \mathrm{F}$

1

2.

3

4

5

6

7

8

9

10

11

12

13

14

15

16

17

18

19

20

21

22

23

24

25

26

27

28

29

30

31

32

\section{-107-x}

$\frac{227}{186}$

186

130

131

288

281

260

231

285

$\frac{276}{255}$

227

277

287

262

284

284

278

265

236

224

149

$\frac{126}{126}$

$\frac{126}{222}$

$\frac{222}{176}$

$\frac{176}{132}$

$\frac{\frac{132}{126}}{225}$

163

$\frac{163}{126}$
10E-SX
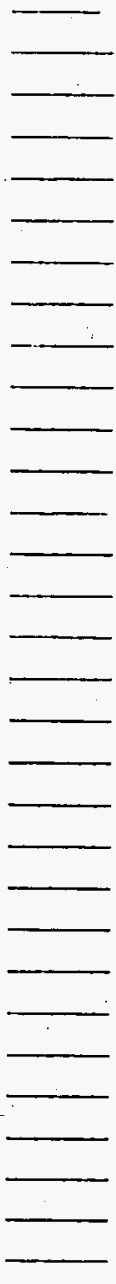
Point Io.

16

17

18

19

20

21

22

23

24

25

26

27

28

29

30

31

32

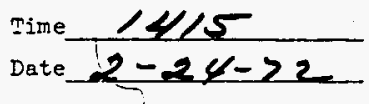

Temperature ${ }^{\circ} \mathrm{F}$

182

130

126

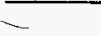

289

281

$26 !$

231

285

276

255

227

278

$\frac{288}{261}$

234

282

$276 / 2$

264

235

222

147

$\frac{126}{126}$

221

$\frac{175}{120}$

126

224

163

126

126 
241-SX SLUDGE PROFIIT TEMPERATURES

TDE 10.15

DATE $3-30-72$

BEST AVAULBLE COPY

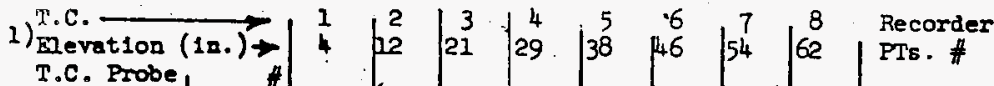
TK-107

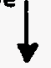

$7 x-108$

$3 x-109$

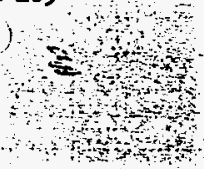

13tos

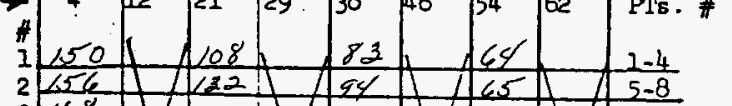

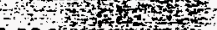

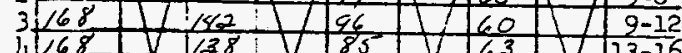

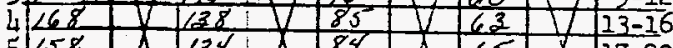

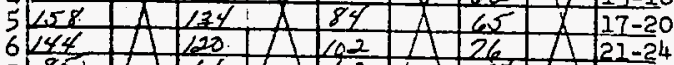

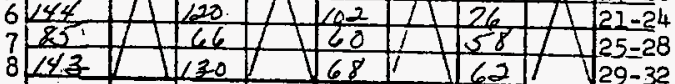

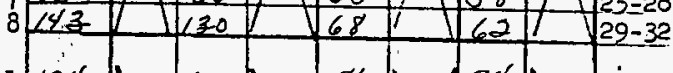

$\left.1 \frac{134}{255}\right)-\left(\frac{100}{13}\right)-\left(\frac{86}{20}\right) \cdot\left(\frac{84}{83}\right)-\frac{i-4}{5-8}$

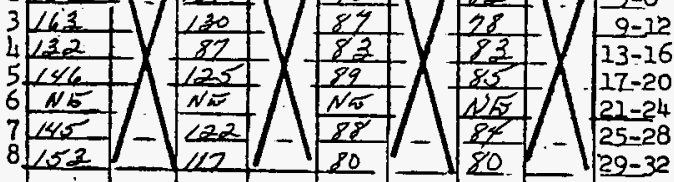

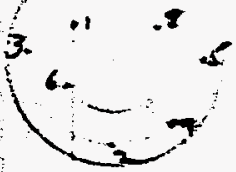

$2 x-110^{\circ}, 301$

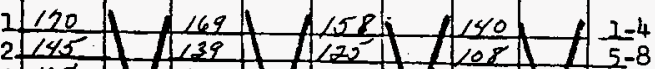

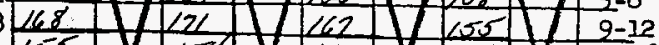

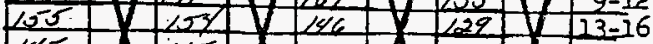

\begin{tabular}{|l|l|l|l|l|l|l|l|l|l|l|l|l|l|}
\hline $17-20$ &
\end{tabular}

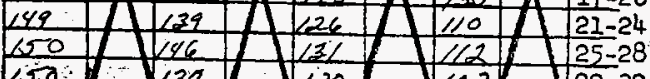

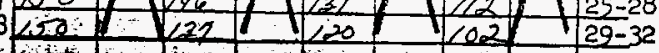

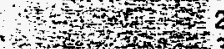

130

3156 129 214195

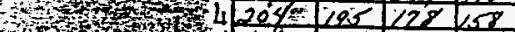

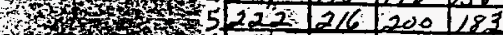

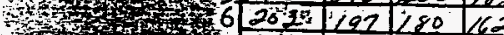

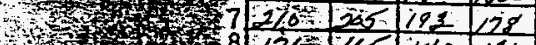

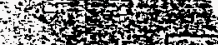

$2 x-117,3,7$,

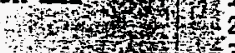

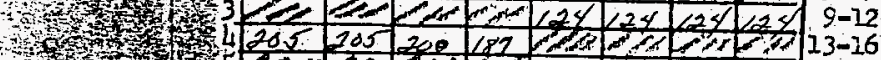

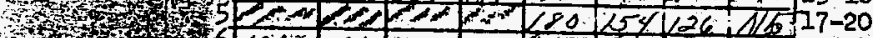

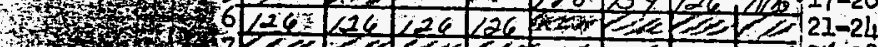

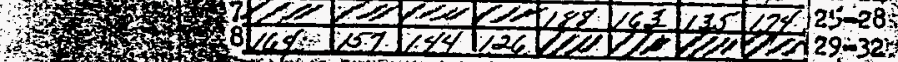

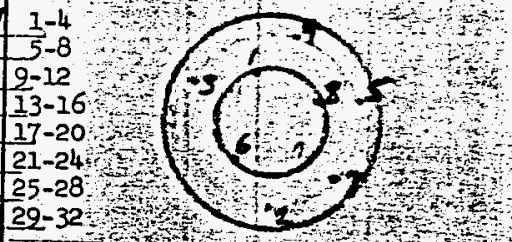

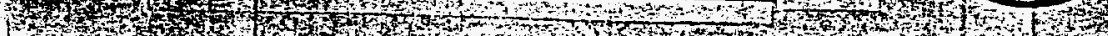

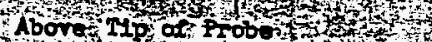

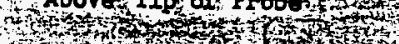

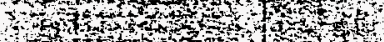

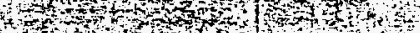

$+3$
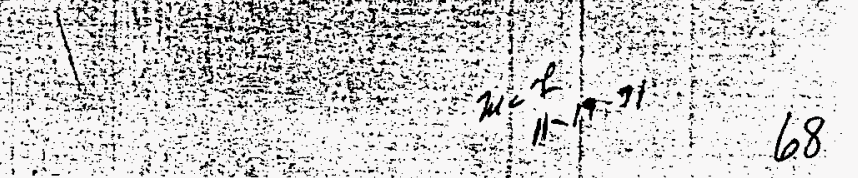
WHC-SO-LAM-DP-232, ReV, 0

$$
3-30-72
$$

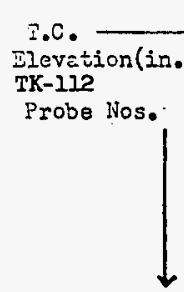

TK- 114

TK-105

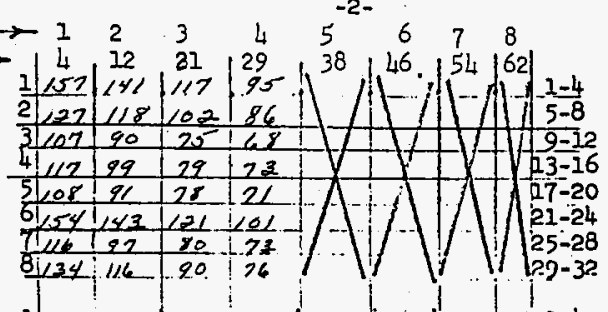

\section{Probe Locations}
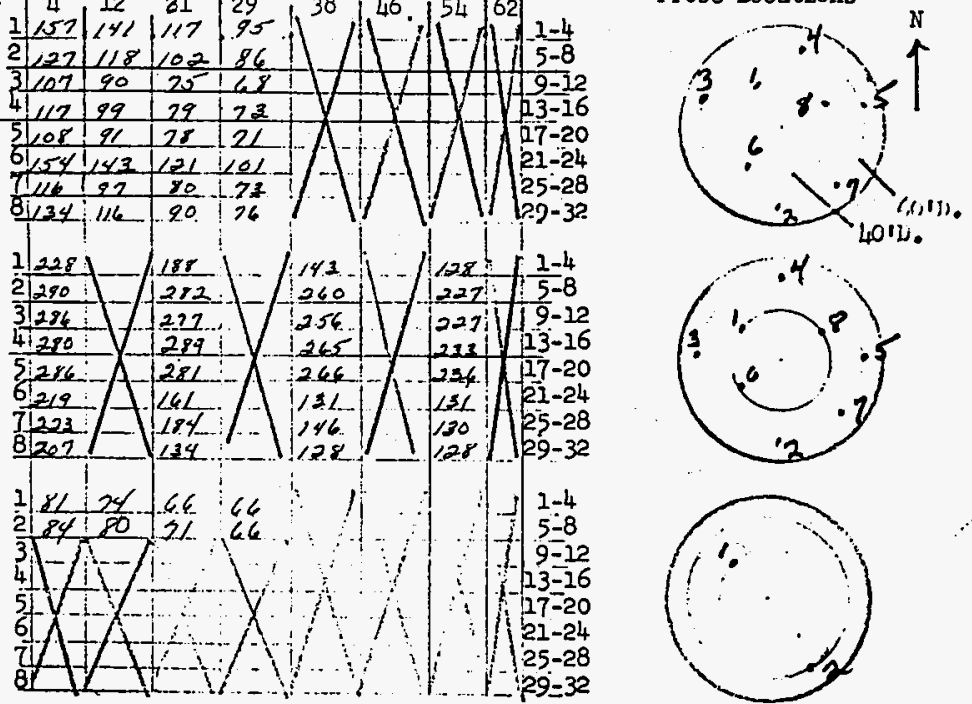

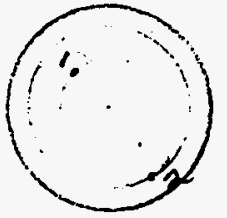

BEST AVALABBIE COPY 
Point lio.

$$
1
$$$$
2
$$$$
3
$$$$
4
$$

21

22

23

24

25

26

- 27

28

29

30

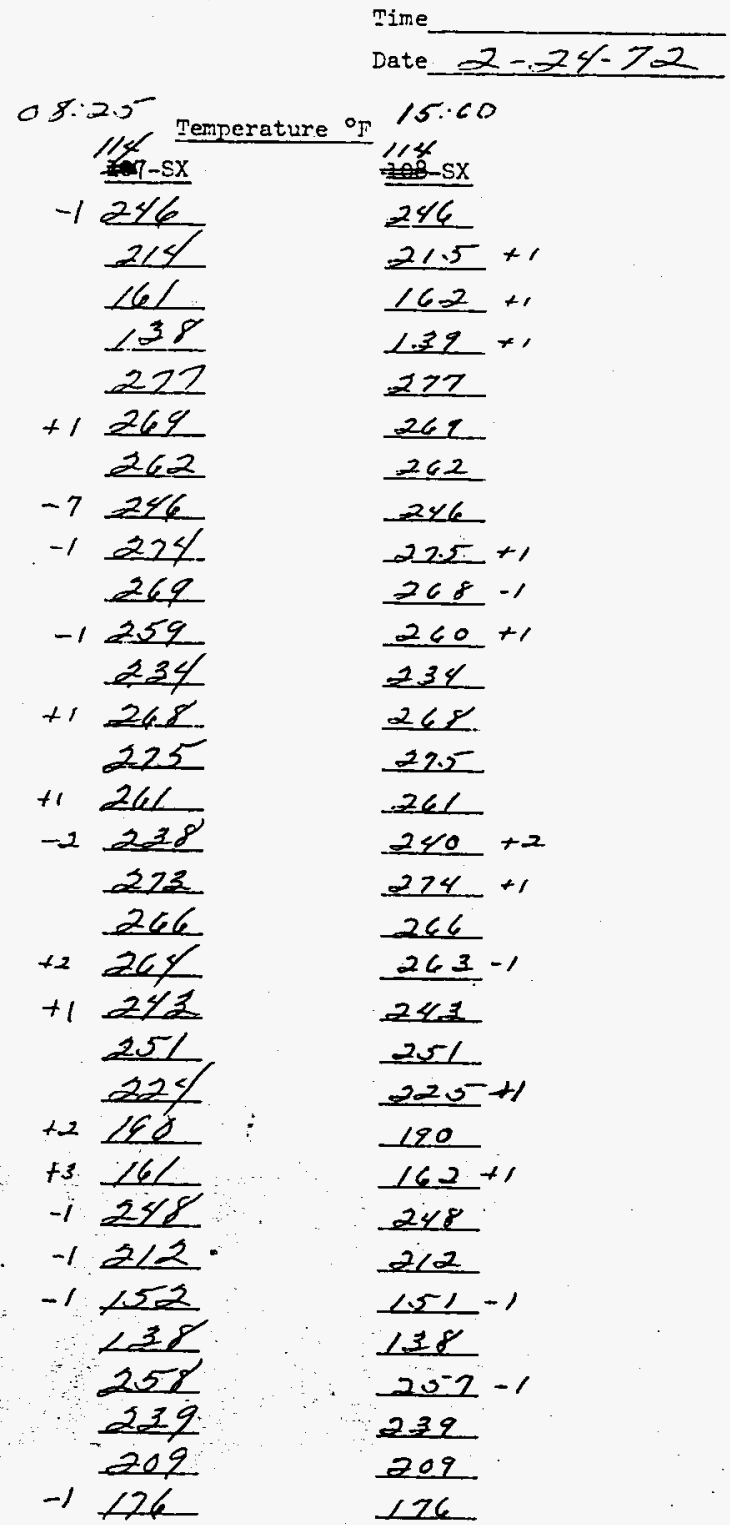




\section{(5.0.P. 200.3.6)}

Point No.

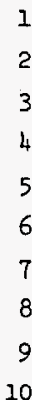

11

12

13

14

15

16

17

18

19

20

21

22

23

24

25

26

27

28.

29

30

31

32

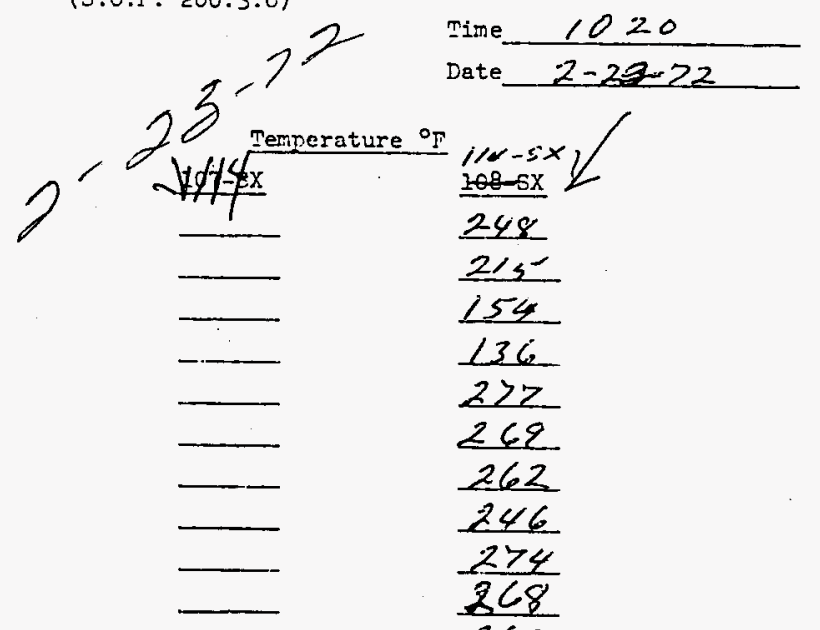

260

234

266

$275^{-}$

257

237

$2 \geq 3$

266

262

243

250

224

185

151

250

$2 / 5$

154

136

257

238

208

175 
Point No.

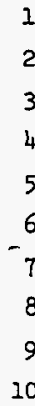

11

12

13

24

15

16

17

18

19

20

21

22

23

24

25

26

27

28

29

30

31

32

\section{(5.0.P. 200.3.6)}

$$
2-23-72
$$

Time -08.40
Time

Date

1,500

$1 / 45$ Temperature $51 / 4 / 5 x$

20

247

214

$\triangle 6 L$

128

228

270

263

246

275

269

260

$-234$

268

276

160

236

273

266

262

242

250

224

187

158

249

$2 / 4$

154

168

257

239

208

176
247

214

161

138

$277-1$

$26 r-2$

$262-1$

$253+7$

$275^{\circ}$

269

260

234

$267-1$

$27.5-1$

260

$240+4$

273

266

262

242

$251+1$

224

$188+1$

158

249

$2 / 3-1$

$153-1$

138

$258+1$

239

$209+1$

$175-1$ 
Point No.

1

2

3

4

5

6

7

8

9

10

11

12

13

14

15

16

17

18

19

20

21

22

23

24.

25

26

27

28

29

30

31

32
Time

Date $1-21-22$

Temperature ${ }^{\circ} \mathrm{F}$ 107-SX
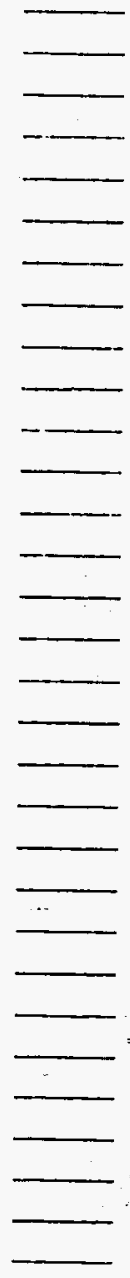

114

100요

250

217

12

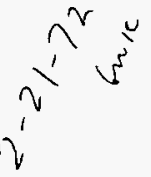

136

227

$\frac{270}{268}$

244

225

268

260

$\frac{234}{268}$

275

263

241

224

267

268

242

251

224

186

144

253

219

158

137

258

240

212

176 
(S.0.P. 200.3.6)

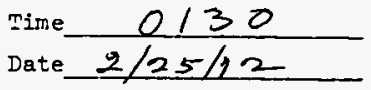

Point No.

1

2

3

4

5

6

7

8

9

10

11

12

13

14

15

16

17

18

19

20

21

22

23

24

25

26

27

28

29

30

31

32

Temperature ${ }^{\circ} \mathrm{T}$

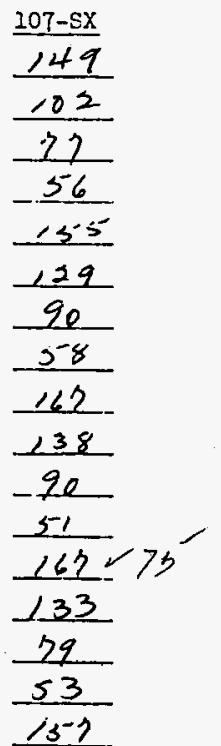

108-SX

130

$-94$

$\frac{81}{98}$

152

127

$\frac{84}{97}$

$160-11$

124

$\frac{83}{28}$

$\frac{78}{129}$

82

25

24

143

122

$\frac{21}{53}$

$1+2$

$\frac{1+2}{112}$

93

20

$\frac{24}{62}$

119

$\frac{82}{36}$

$\frac{26}{N 6}$

$N C$

$N G$

$N G$

140

55

53

140

126

$<2$

55

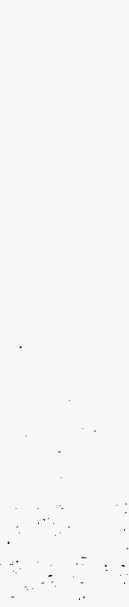

$\frac{115}{82}$

$\frac{77}{150}$

10 


Time $\frac{0 / 40}{2 / 25 / 22}$

Point Io.

20

21

22

23 24

25

26

27 28 29 30 31 32
Temperature ${ }^{\circ}$ I09-sX

$174 \times 9464157-247$

172

160

$-139$

148

142

122

108

172

174

$-168$

156

$\frac{152}{156}$

147

$\frac{128}{147}$

$\frac{147}{1+6}$

142

134

144

140

$\frac{140}{127}:$

$\frac{12}{112}$

160

143.

132

111

$\angle 3-2$

142

124

104
$11^{2}-114$

$140 \quad 215$

112163

93/38

$126 \quad 277$

117268

$-100262$

$83=49$

$-103 \quad 275$

86 269

$72 \quad 260$

L2 233

115268

95276

74262

$64 \quad 239$

103273

86262

$72 \quad 263$

62243

130251

140225

$119 \quad 191$

$99 \quad 164$

113.242

93212

25,30

द4 139

$124=258$

112238

87.269

72116 


Time $-\frac{0 / 30}{2 / 24 / 22}$

Point No.

$$
1
$$

21

22

23

24

25

26

- 27

28

29

30

31

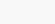

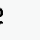

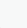

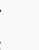

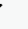

(

7

8

Temperature ${ }^{\circ} \mathrm{F}$

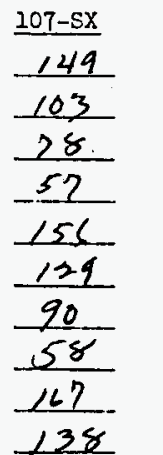

138

$-90$

$\frac{50}{167} \cdot 75$

134

29

$-53$

157

$\frac{124}{75}$

57 .

143

113

96

70

$\frac{70}{80}$

62

55

$\frac{53}{54}$

$\frac{24}{146}$

126

42

$s-5$
$108-5 X$

130

84

80

28

154

226

$\frac{54}{26}$

$\frac{26}{160}-\geqslant$

126

83

27

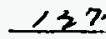

82

$\frac{25}{15}$

143

119

$\frac{5 s}{2 s}$

$x$

$\frac{x}{x}$

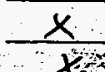

$\frac{\frac{139}{113}}{\frac{80}{25}} \frac{148}{\frac{111}{23}}$ 


\section{(5.0.P. 200.3.6)}

Time $0 / 40$

Point Ho.

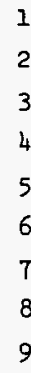

10

11

12

13

14

15

16

17

18

19

20

21

22

23

24

25

26

27

28

29

30

31

32
109 Temperature ${ }^{\circ} \mathrm{T}$

207

1242

172

160

$-\frac{138}{148}$

142

126

108

172

$\frac{174}{168}$

156

157

156

147

127

147

146

142

134

149

140

$126:$

110

150

$\frac{147}{132}$

110

152

142

$\frac{124}{104}$
$112-5 x$

$100-8 x$

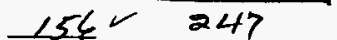

$140 \quad 214$

$112 \quad 162$

$95 \quad 138$

$126 \quad 277$

117270

$160 \quad 262$

84248

164274

56269

260

235

268

219

$\begin{array}{ll}96 & 262 \\ 24 & 238\end{array}$

$\frac{24}{64} 273$

$\frac{64}{103} \quad 267$

$8<\quad 2<3$

$13 \quad 243$

$62 \quad 252$

224

$130 \quad 188$

$142 \quad 161$

119248

$99>13$

$108 \cdots, 51$

$93 \quad 138$

$-25 \quad 257$

$64 \quad 238$

$\frac{130}{113} 209$

$\frac{113}{88}=176$ 


Time $\frac{0 / 30}{2 / 23 / 122}$

Point No.

1

2

3

4

5

6

7

8

9

10

11

12

13

14

25

16

17

18

19

20

21

22

23

24

25

26

27

28

29

30

32

32

Temperature ${ }^{\circ} \mathrm{F}$

$107-\mathrm{Sx}$

149

102

32

$-52$

156

129

89

$-5-8$

$168 x 76$

138

$-90$

52

167

134

79

54

152

130

29

58

143

112

95:

$\frac{10}{19}$

62

56

54

140

$\frac{-126}{62} \frac{56}{-56}$
108-5X

130

94

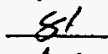

35

152

127

84

22

$-160 x \geqslant$

126

53

28

127

$\frac{82}{26}$

143

119

82

36

$N c$

NC

$N E$

$\frac{N C}{10}$

140

117

$8 \alpha$

22

149

$\frac{\frac{112}{24}}{24}$ 


Time $\frac{0 / 50}{2 / 22 / 22}$

Point No.

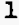

2

3

4

5

6

7

8

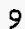

10

11

12

13

14

15

16

17

18

19

20

21

22

23

24

25

26

27

28

29

30

31

32
Temperature ${ }^{\circ} \mathrm{F} / 12$

$\underline{\log -S \mathrm{X}}$

$174 \times 79$

173

160

$-139$

149

142

122

109

172

125

$-\angle L q$

156

$<57$

152

142

127

145

146

142

134

150

140

127

111

151

142

132

$\frac{111}{15}$

153

$\angle 4 z$

124

105
$2-5 x$

$152 \times 69$

140

112

94

127

112

100

54

$-104$

86

71

42

116

96

24

65

103

87

13

63

150

141

$1 / 9$

$=99$

113

82

75

65

130

112

87

73
$114-5 x$

247

2,3

159

138

277

261

262

247

274

268

260

234

267

$2 \pi 6$

260

187

273

267

$2<2$

242

251

224

$18 ?$

157

249

2,4

153

138

258

739

209

176 
WHC-SD-LNA-DP-232, ReV. 0

241-S2 SLUDGE PROFIIE TDMPERAMURES

(5.0.P. 200.3.6)

Time $\frac{0 / 30}{2 / 22 / 32}$

Point No.

1

2

3

4

5

6

7

8

9

10

11

12

13

14

15

16

27

18

19

20

21

22

23

24

25

26

27

28

29

30

32

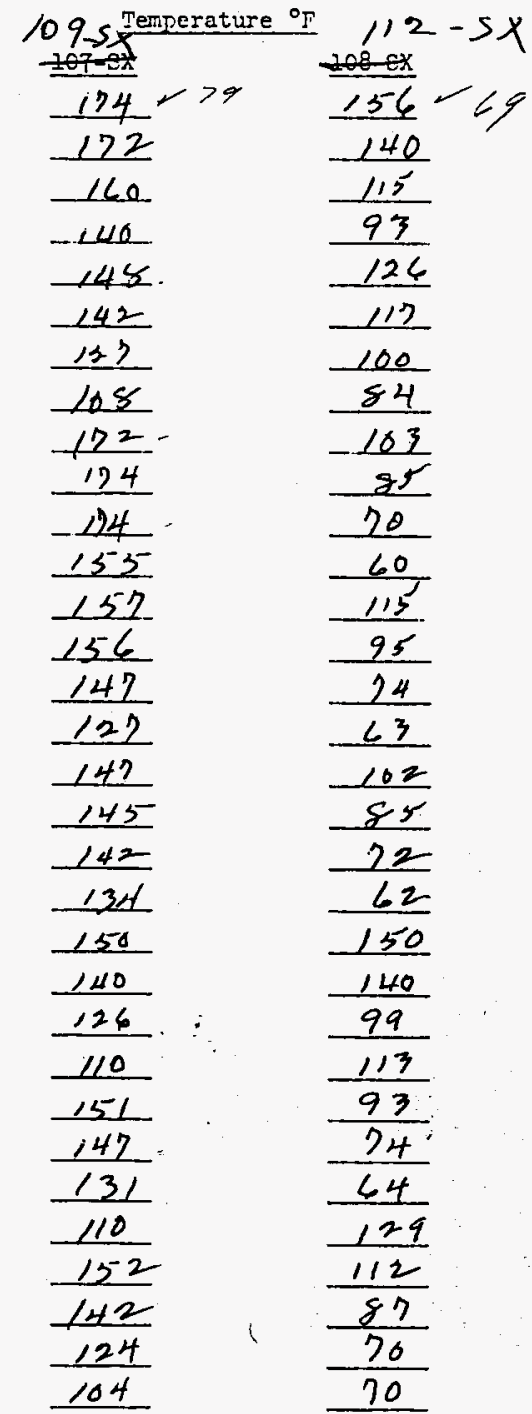

80 
Time

Date

$\frac{0,40}{1 / 22 / 32}$

\section{Point No.}

1

2

3

4

5

6

7

8

9

10

11

12

13

14

15

16

17

18

19

20

21

22

23

24

25

26

27

28

29

30

31

32

Temperature ${ }^{\circ} \mathrm{F}$

\section{7-5X}

149

102

26

$\frac{54}{156}$

156

128

89

$\frac{57}{167}+75$

138

$-89$

50

162

$-133$

29

53

151

130

72

52

143

116

$\frac{116}{95}:$

28

79

$+060$

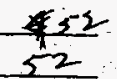

52

140

125

61

55
108-sX

130

93

80

27

$-152$

126

-83

$\frac{76}{-160}+7$

125

82

77

126

SO

25

25

143

118

80

74

N 6

$N C$

$N 6$

$N c$

140

115

80

23

73

148

$\frac{110}{12}$ 
(S.0.P. 200.3.6)

Time $\frac{0,30}{3 / 22}$

Point Iio.

1

2

3

4

5

6

7

8

9

10

11

12

13

14

15

16

17

18

19

20

21

22

23

24

25

26

27

28

29

30

31

32
Temperature ${ }^{\circ} \mathrm{F}$ 107-SX

$\frac{\frac{151}{110}}{\frac{85}{-47}}$

158

133

$\frac{98}{28}$

$\sqrt{-}$

$\sqrt{1}$

143

$-\angle 00$

62

169.

$-135$

$\frac{88}{64}$

158

135

82

62

144

, 26

103

80

82

20

65

$-3$

143

131

$\frac{22}{65}$
SX

108-5x $1045 x, 1125 \times 1145 i$ \begin{tabular}{l|l|l|l}
135 & 172 & 152 & 228 \\
& 101 & 142 & 186
\end{tabular}

\begin{tabular}{|c|c|c|c|}
\hline 0 & & & \\
\hline 58 & 154 & 118 & 人々5 \\
\hline 83 & 140 & 98 & 124 \\
\hline 156 & 146 & 123 & $\begin{array}{l}285 \\
280\end{array}$ \\
\hline 132 & 140 & 178 & \\
\hline 93 & 126 & 102 & 261 \\
\hline 86 & 108 & 88 & 239 \\
\hline
\end{tabular}

$\checkmark$
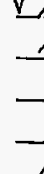

133

89

8

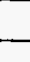

$\frac{147}{125}$

$\frac{125}{88}$

83

NG

147

$\sqrt{ }$

$i>2$

108

92

$7 ?$

68

135

155

11

153

145

100

80

$127 \mathrm{~h} 2$

144107

143

92

139

133

18

30

148

138

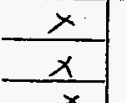

$\frac{x}{x}$

12

108

$\frac{143}{122}$

$\frac{122}{88}$

$\sqrt{ }$

85

$-50$

145

130

112

150

$p$

118

$4 / 38$

150

14

120

100

115

97

81

73

133

13

116

285

236

255

227

277

287

266

234

282

273

264

236 .

236

138

124

125

223

173

> 4

124

\begin{tabular}{r|l|l}
82 & 92 & 124 \\
102 & 38 & 124. \\
\hline
\end{tabular}

\begin{tabular}{r|l|l}
82 & 92 & 124 \\
102 & 38 & 124. \\
\hline
\end{tabular} 


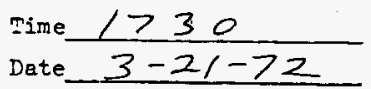

Point No.

$$
1
$$

2

3

4

5

6

7

8

9

10

11

12

13

14

15

16

17

18

19

20

21

22

23

24

25

26

27

28

29

30

31

32
$1 / 4$ Temperature ${ }^{\circ}$

10T-SX 108-SX

228

$\frac{180}{124}$

288

280

260

$\frac{260}{235}$

285

276

255

226

277

287

260

$\frac{260}{234}$

283

277

264

235

231

134

$\frac{124}{124}$

223

$171=$

$\frac{17}{124}$

124

220

161

$\frac{161}{124}$

124 
24I-SX SLUDGE PROFILE TIMTERATURIS

(S.0.P. 200.3.6)

Point No.

1

2

3

4

5

6

7

8

9

10

11

12

13

14

15

16

17

18

19

20

21

22

23

24

25

26

27

28

29

30

31

32
1030

$1 / 4 / 5$ femperature ${ }^{\circ} \mathrm{F}$

$200-x$

228

$\angle 20$

124

224

28

$28 \%$

$\frac{261}{232}$

28

226

253

227

228

282

24

232

22

277

211

235

233

135

$2+4$

124

212

271

124

124

$\frac{12}{21}$

221

124

124
108-SX

Time

Date $3-2<-72$ 


Dime $\frac{0100}{3 / 24 / 22}$

Temperature ${ }^{\circ} \mathrm{F}$

Point 10.

1

2

3

4

5

6

7

8

9

10

11

12

13

14

15

16

17

18

19

20

21

22

23

24

25

26

27

28

29

30

31

32

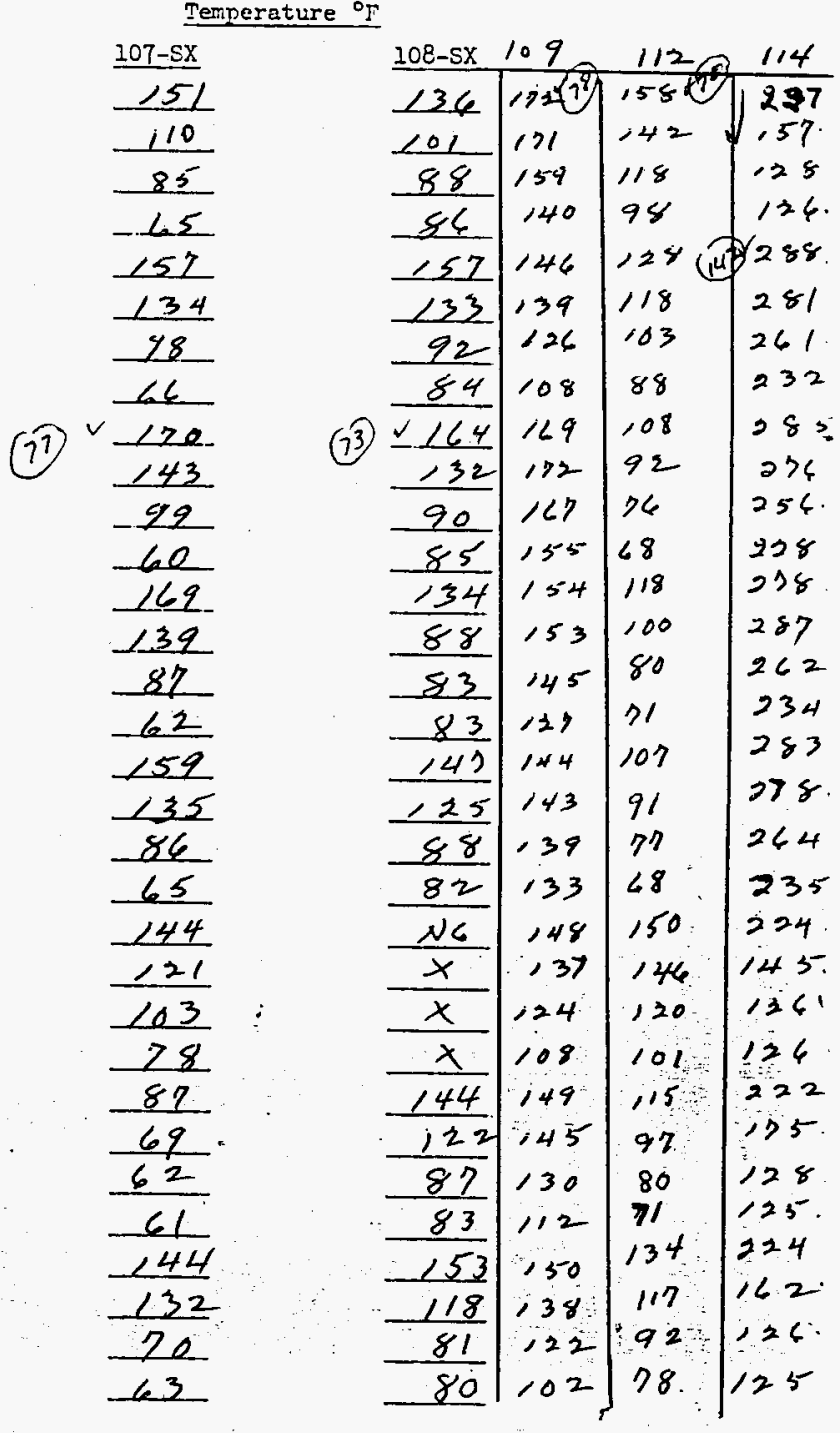

85 


\section{(S.0.P. 200.3.6)}

Point No.

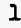

2

3

4

5

6

7

8

9

10

11

22

13

14

15

16

17

18

19

20

21

22

23

24

25

26

27

28

29

30

31

32

180
$4-4-12$

Time 1740

Date $3-23-7=$
$1 / 4$ Temperature ${ }^{\circ} \mathrm{F}$

-SX

276

126

126

288

280

265

业2

285

275

260

227

278

282

272

238

283

278

268

236

224

$14 Q$

126

126

222

174

127

126

224

$\frac{224}{162}$

126

125

108-SX
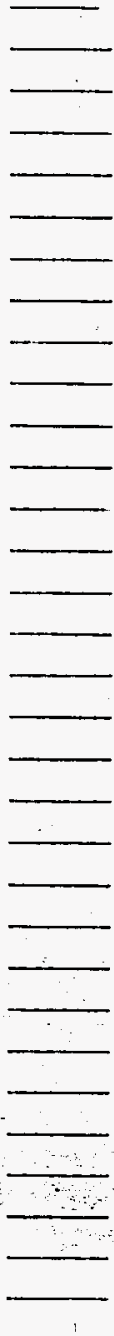
241-SX SLUDGE PROFIIE TEMPERATURES TIM $\angle 1400$ DATE $2-33-72$

\section{BEST AVALABLE COPY}

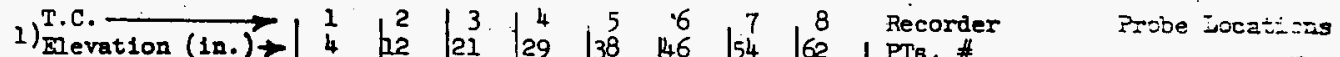
TK-107

$\mathrm{TK}-108$

TK-109

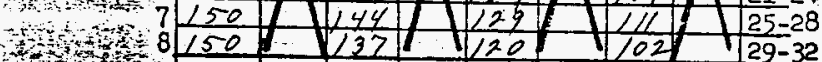

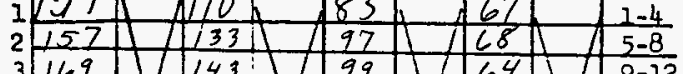

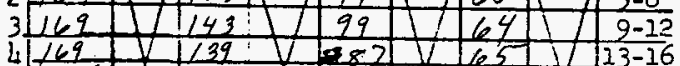

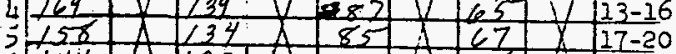

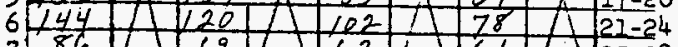

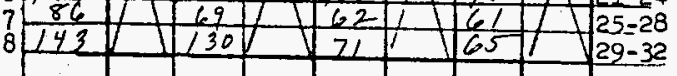

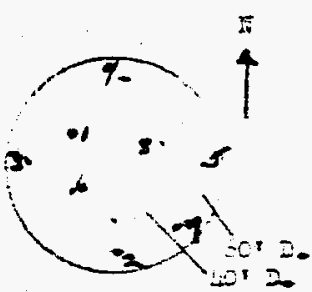

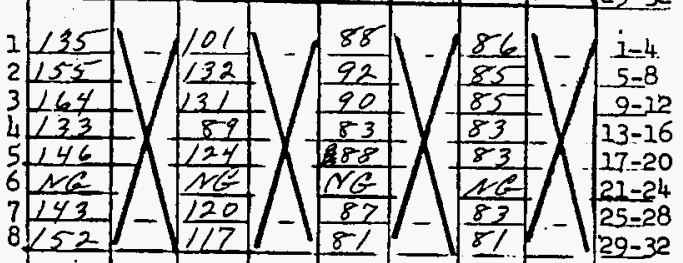

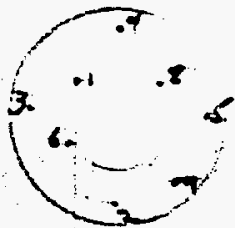

$1 / 21 / 1701 / 49$

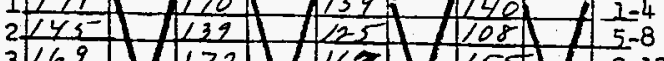
$3 / 69+172) / 1621 / 155) / 9-12$

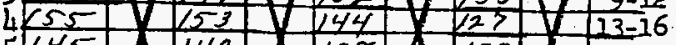

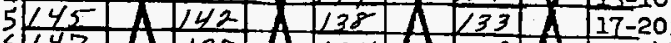

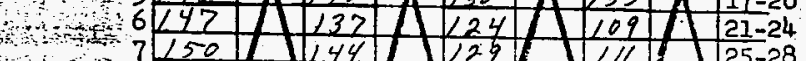

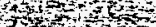

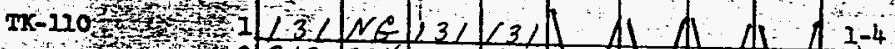

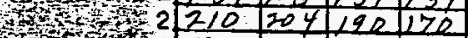
\begin{tabular}{ll|l|l|l|l|l|}
236 & 230 & 215795 \\
\hline
\end{tabular} $\begin{array}{ll}3 & \end{array}$ \begin{tabular}{ll|l|l|l|l|l|}
\hline 21 & 215 & 192 & 181 \\
\hline
\end{tabular}

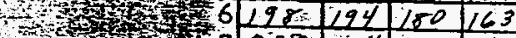

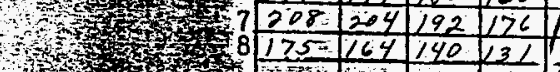

TK-211 J T I

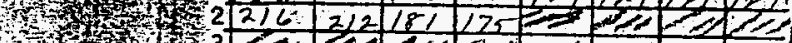

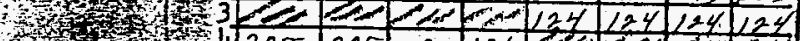

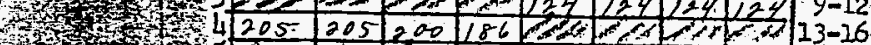

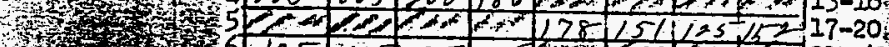

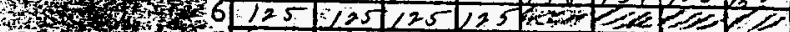

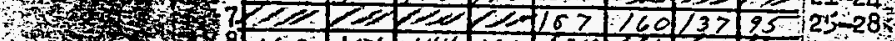

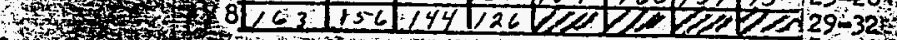

47 , $13 x$

ABore il 3 of Probe

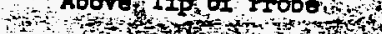

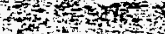

$+4+3<2+4$

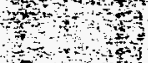

$+3+4$ 
BEST AVALABBIE COPY

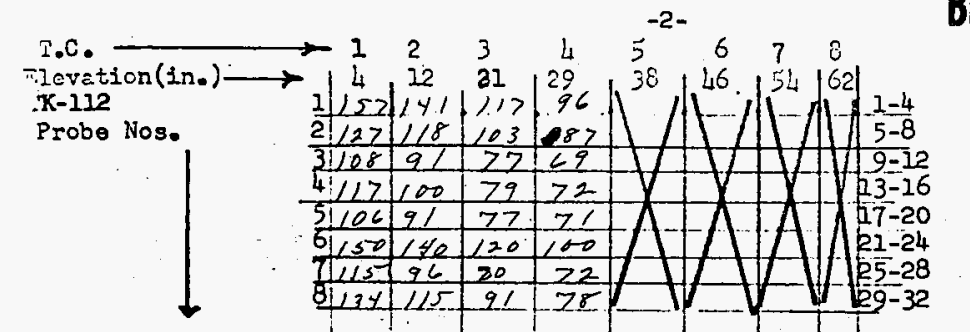

Probe jocazin:as =

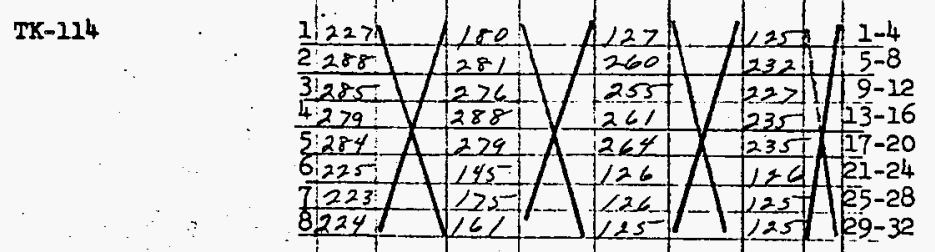

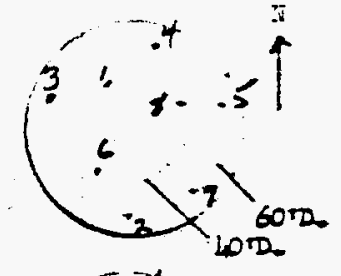

$-24$

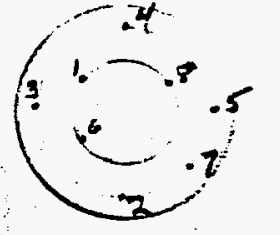

TK-105
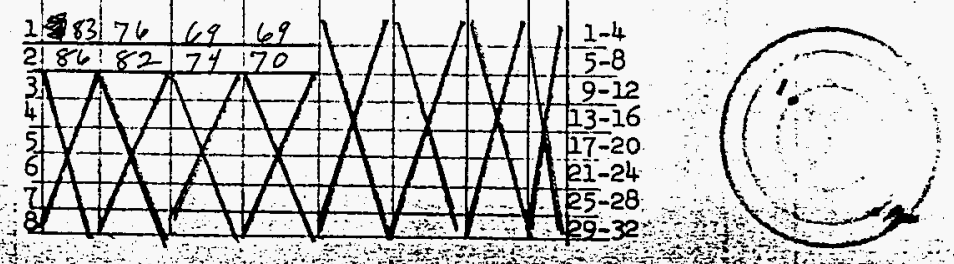


\section{1-SX 3IUDGE COOLER FROCEDURE}

Dite $3 / 23 / 22$ Time of Reedinss Dry $_{\text {Sinky }}$ swing $/ 730$ G.Y. 0210

Underground Tnnks

Outlet Air Temperatures $\left({ }^{\circ} \mathrm{F}\right)$

TK No. TR-5X-2 Pt\#

$\begin{array}{ll}107 & 17 \\ 108 & 18 \\ 109 & 19 \\ 110 & 20 \\ 111 & 21 \\ 112 & 22 \\ 114 & 23\end{array}$

Maximum Sludge Temperatures

$\left({ }^{\circ} F\right)$ at $4 "$ from t1p of probe.

Record the maximum Temperature

for each group of 8 T.C.\#'s

Tk No. Recorder Point wo.

109 TR-SX-1 1 to 8

110 TR-SX-1 9 to 16

1.11 TR-SX-1 17 to 24

112 TR-SX-2 1 to 8

114 TR-SX-2 9 to 16

\section{Air Heater}

Steam Supply (psig)

Inlet TI-1-I E (OF)

outlet North $(0)$ South $(0)$

Outlet TI-l-2-W (OF)

\section{FYlbers}

Air Inlet $T^{\circ} \mathrm{C}(\mathrm{TR}-\mathrm{SZ}-1)$

North dp(in.W.G.) (aI-1-1)

$a_{0}=(\mathrm{dI}-1-2)$

Center dp(In.W.G.) (dI-I- 3 )

a $(\mathrm{aI}-1-4)$

South dp(in.W.G.) (dI-1-5)

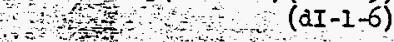

Seal Pot L quid Level(in.W.G.)

Exhaust Fans?

Fan Operating (N \&/or $s)$

Stack Flow (FPM x 1000)

Stack ${ }^{\circ} \mathrm{F}=$

Stack Radiation (RR-SX-1)

Fin \& Chart 1

$\operatorname{Pange}\left(10,10^{2}, 10^{3}, 10^{4}\right)$

Multiplier $(1 X, 2 X, 5 X, 10 X)$

operating (yes, No)
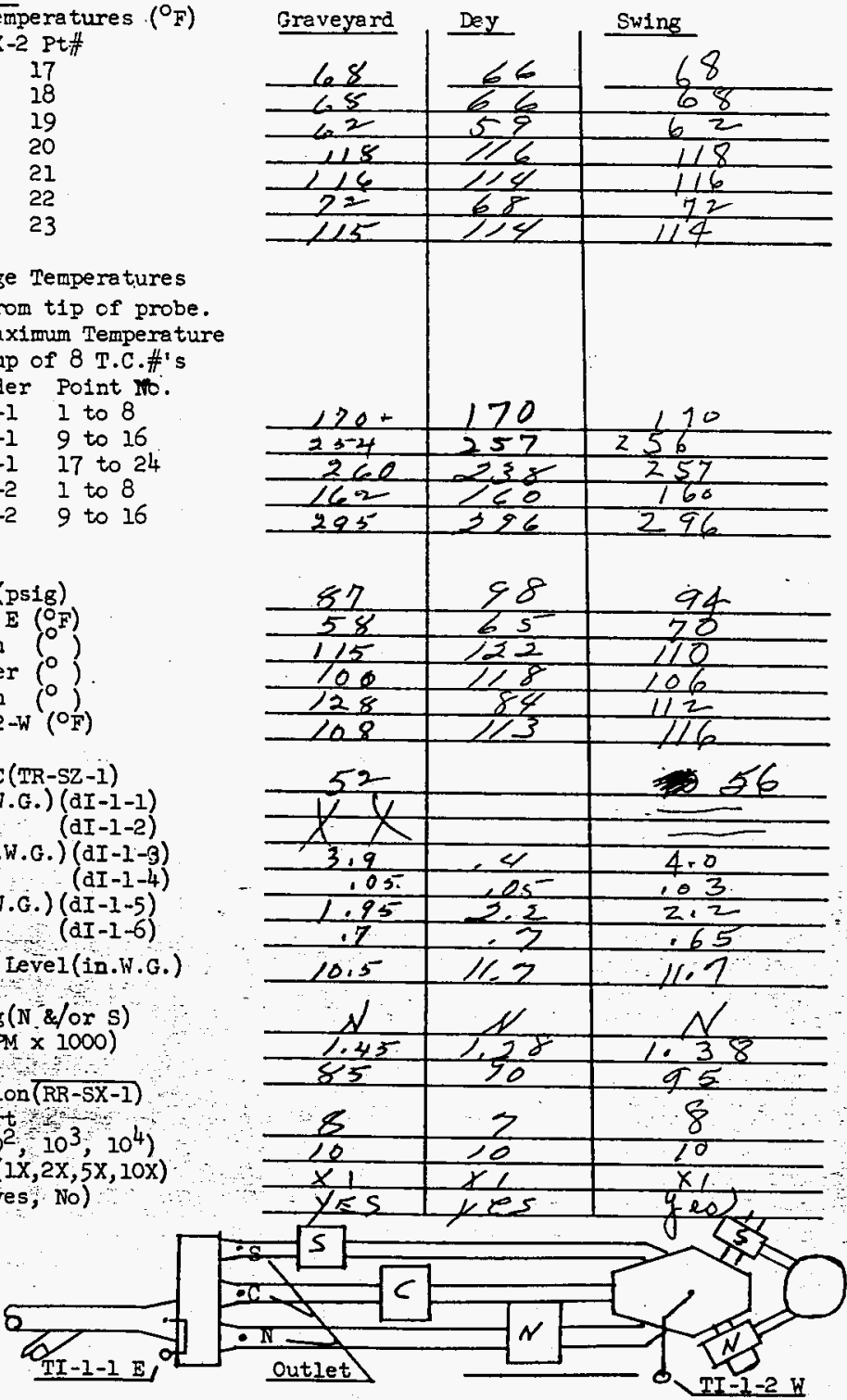


Time $-3 / 23 / 72$

Point No.

$$
1
$$$$
2
$$$$
3
$$

4

$$
5
$$$$
6
$$$$
7
$$$$
8
$$$$
9
$$

16 17

18

19

20

21

22

$-23$

24

25

26

27

28

29

30

$$
31
$$

32

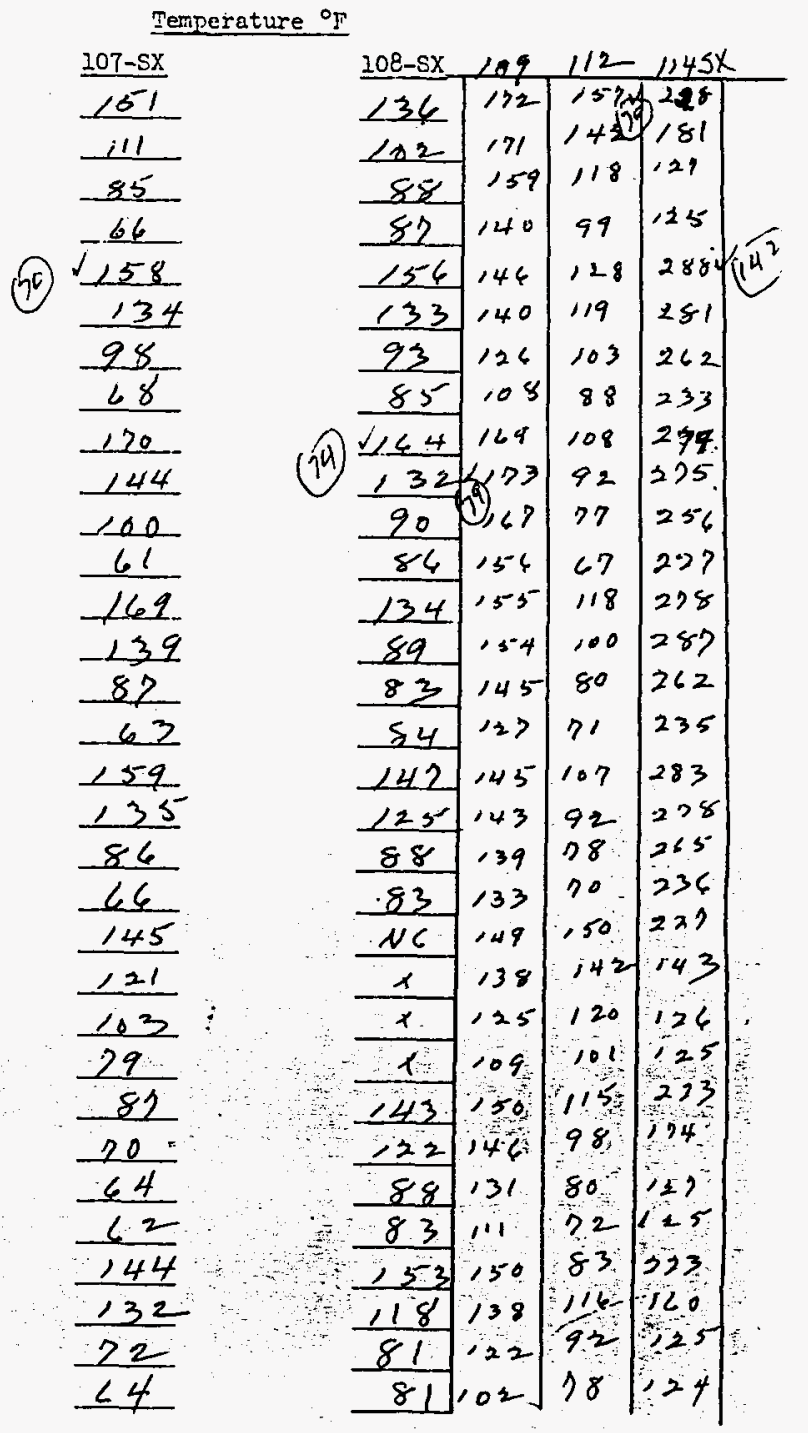


241-SX SLUDGE PROFILE TENPEPATURES (S.0.P. 200.3.6)

Point No.

1

2

3

4

5

6

7

8

9

10

11

12

13

14

15

16

17

18

19

20

21

22

23

24

25

26

27

28

29

30

31

32

$$
\begin{aligned}
& 4-12 \\
& A-1600-2400 \\
& \text { Time } \frac{1930}{3-2 z-2 z}
\end{aligned}
$$

114 Temperature ${ }^{\circ} \mathrm{F}$

z-SX

275

176

126

125

228

278

265

231

277

275

255

226

276

285

$\pm 60$

235

280

$\frac{276}{265}$

226

145

125

125

$\frac{222}{175}$

$\frac{115}{125}$

125

220

$\angle 60$

125

125

108-SX
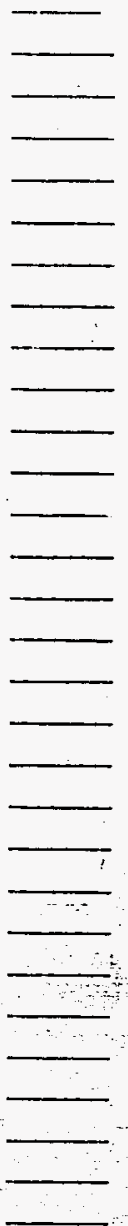


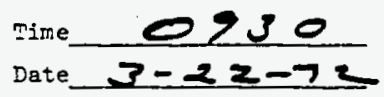

Point 10.

1

2

3

4

5

6

7

8

9

10

11.

12

13

14

15

16

17

18

19

20

21

22

23

24

25

26

27

28

29

$\therefore \quad 30$

31

32.
$1 / 4$ moperature ${ }^{\circ} \mathrm{F}$

101-2x- 108-SX

238

120

$\angle 25$

221

282

220

202

725

226

$\frac{25}{225}$

272

$=87$

260

234

223

227

264

235

232

12

124

142

122

123

124

$\angle 24$

160

124

122 
241-SX SLUDCE PROFILE TINIPEATURES

(S.0.P. 200.3.6)

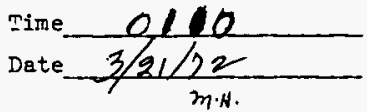

Point No.

1

2

3

4

5

6

7

8

9

10

11

12

13

14

15

16

17

18

19

20

21

22

23

24

25

26

27

28

29

30

31

32

Temperature ${ }^{\circ}$

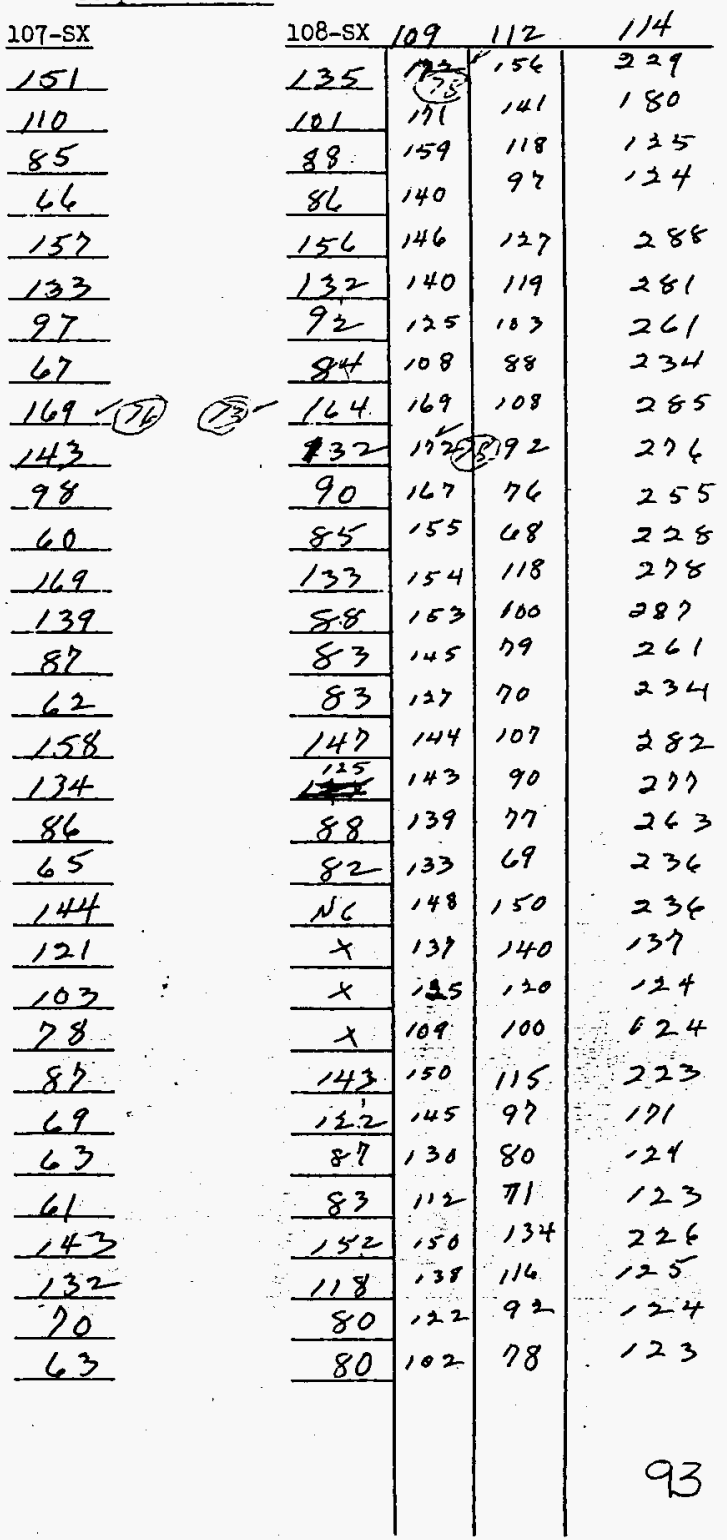


Point No.

1

2

3

4

5

6

7

8

9

10

II

12

13

14

15

16

17

18

19

20

21

22

23

24

25

26

27

28

29

30

31

32
Time $\frac{17 / 5}{3-20-72}$

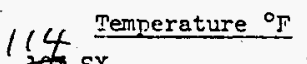

- $5 x$

229

180

124

122

288

280

260

235

285

275

2.55

228

278

287

260

235

282

277

$\frac{277}{264}$

235

237

138

122

$\frac{122}{224}$

224

170.

$\frac{170}{122}$

$\frac{122}{121}$

230

$\frac{230}{125}$

121

121

108-5X
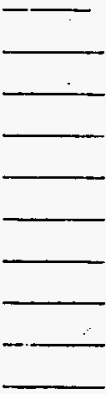

$+$
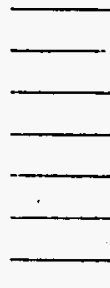

$\longrightarrow$
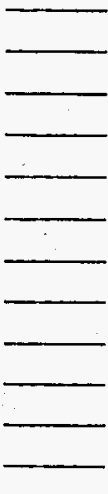


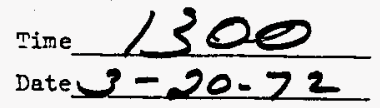

Point No.

1

2

3

4

5

6

7

8

9

10

11

12

13

14

15

16

17

18

19

20

21

22

23

24

25

26

27

28

29.

30

31

32
$1 / 4 / 5$ mperature ${ }^{\circ}$

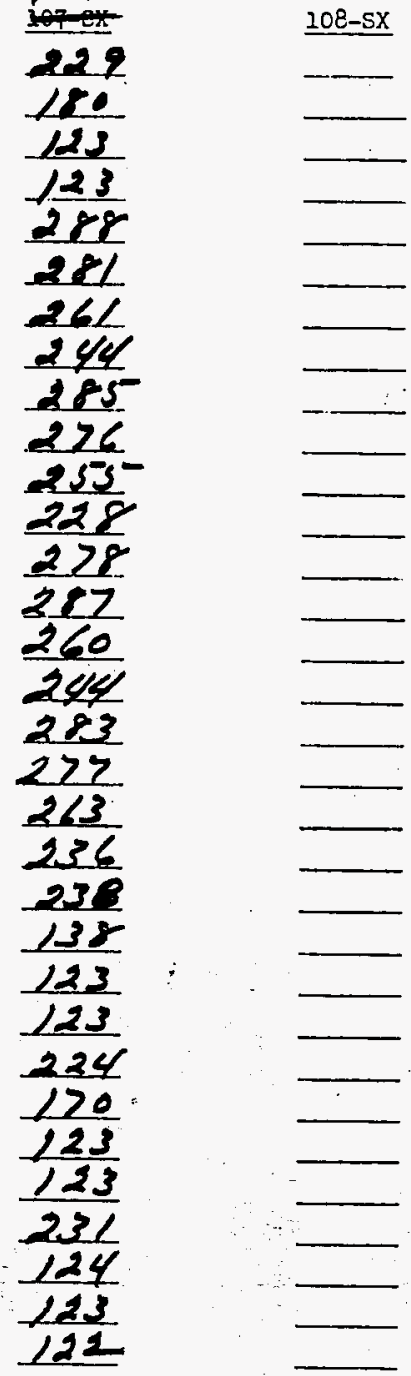


241-SX SLUDGL PROFILE TDMPERATURIS

(5.0.P. 200.3.6)

Point lio.

$$
\text { Time } \frac{0100}{3 / 20 / 22}
$$

Temperature ${ }^{\circ} \mathrm{F}$

$112.5 x \quad 107-5 x \quad 109-5 x \quad 108-5 x \quad 114-5 x$
$156^{\prime}|5| \quad 122 \quad 135 \quad 228$ 140 110 $171.101 \quad \frac{180}{180}$ $118-85-159-88-123$ $98-66-140-\frac{16}{86}-\frac{123}{143}$ $127+152-146+55 \div 288 \omega$ $119+133+132-280$ $\frac{83}{67}-\frac{108}{169} \frac{84}{23}-235$ 108169621692425 $92-143591123 \pi$ $\frac{92}{77} \frac{143(11232}{-99}-\frac{132}{968}-\frac{226}{90}-\frac{256}{258}$ 236 $68-60-155-85-228$ $118-169-155-133-228$ $160-138-154-88-282$ $79-\frac{138}{88}-145-\frac{88}{83}-\frac{262}{261}$ $71-62-\frac{145}{-22}-\frac{83}{83}-235$ $107-158-145-144-282$ $\frac{92}{78} \frac{134}{86}-\frac{144}{140}$ $\frac{69}{150}-\frac{65}{144} \frac{138}{148}$

$\frac{125}{88} \frac{217}{263} \frac{337}{242}$

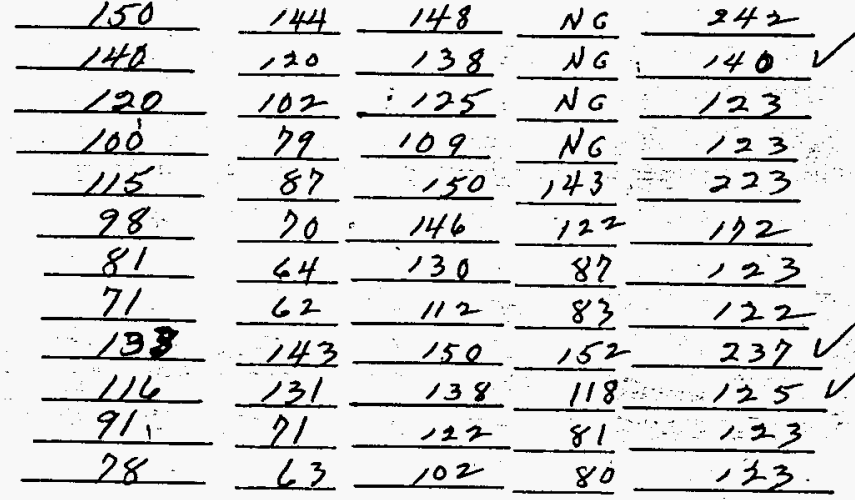


24I-SX SLUDGI PROFILE TDIPERATURES

Point No.

1

2

3

4

5

6

7

8

9

10

11

12

13

14

25

16

17

28

19

20

21

22

23

24.

25

26

27

28

29

30

31

32
(5.0.P. 200.3.6)

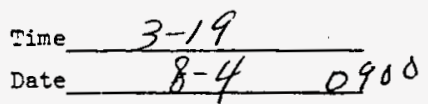

$114-8 x_{\text {Temperature }}^{\circ} \mathrm{F}$

$$
\text { roI }=5 x
$$

$108-5 X$

230

180

122

122

288

280

260

235

285

$\frac{225}{455}$

229

227

286

260

235

282

276

242

236

247

$10 \frac{2}{2}$

$1 \varepsilon q$ :

$\frac{122}{224}$

169.

120

120
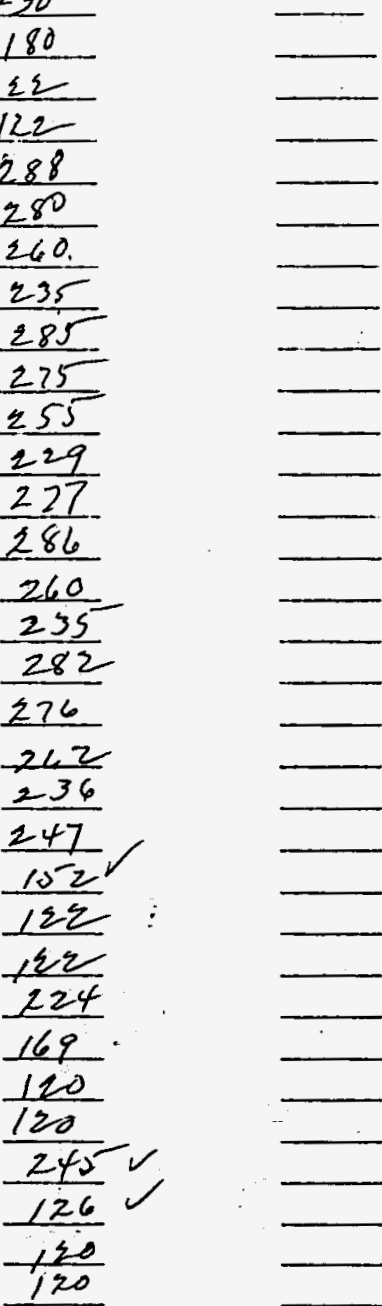

$\longrightarrow$
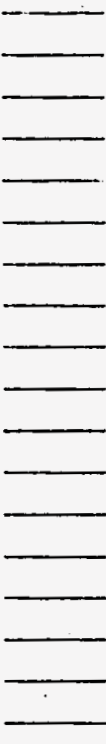
Point No.

$$
1
$$$$
2
$$$$
3
$$$$
4
$$$$
5
$$$$
6
$$$$
7
$$$$
8
$$$$
9
$$$$
10
$$

11

12

13

14

21

22

23

24

25

26

. 27

28

29

30

31

32

$$
114-5 x
$$

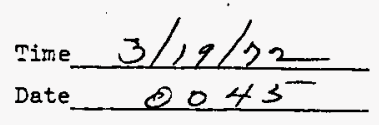

Temperature ${ }^{\circ} \mathrm{F}$

$207 \& x$

230

180

122

122

255

$25 \%$

262

236

$\frac{254}{214}$

$-255$

228

231

282

261

235

$2 \& 2$

212

$2<3$

23

250

170

$122:$

122

224

170

122

121

$\frac{252}{131}$

122

122

n) 1 .

202
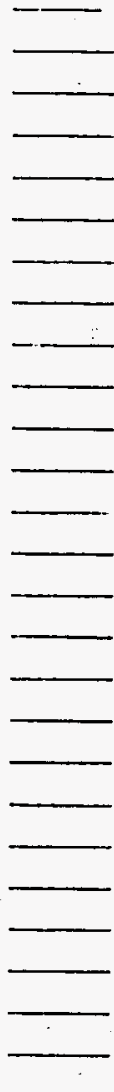


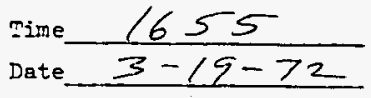

Point No.

\section{1}

114 Temperature :

$108-5 x$

230

180

$\frac{120}{120}$

287

280

260

$\frac{235}{285}$

285

275

255

229

278

286

260

$\frac{260}{235}$

282

276

263

235

245

145

$\frac{140}{120}:$

120

225

$\frac{225}{170}$.

120

120

241.

$\frac{245}{120}$

$\frac{120}{120}$ 
241-SX SLUDGE PROFIIE TWMERATURES

(5.0.P. 200.3 .6$)$

Time $\frac{1715}{3-18-72}$

Point No.

1

2

3

4

5

6

7

8

9

10

11

12

13

14

15

16

17

18

19

20

21

22

23

24

25

26

27

28

29

30

31

32
$1 / 4$ Tenperature ${ }^{\circ} \mathrm{T}$

ET-SX

230

$\frac{180}{120}$

120

287

280

260

235

285

275

255

229

272

286

260

235

282

276

262

236

253

$\frac{253}{175}$

120

120

225

170

$\frac{120}{120}$

231

$\frac{231}{147}$

120.

120
$108-5 X$
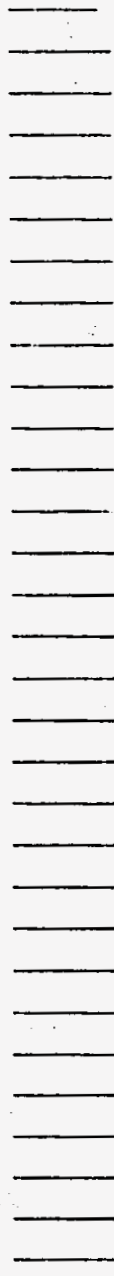
(5.0.P. 200.3.6)

Time $\frac{1032}{3-10 \cdot 72}$

Point No.

1

2

3

4

5

6

7

8

9

10

11

12

13

14

15

16

17

18

19

20

21

22

23

24

25

26

27

28

29

30

31

32
Temperature ${ }^{\circ} \mathrm{F}$

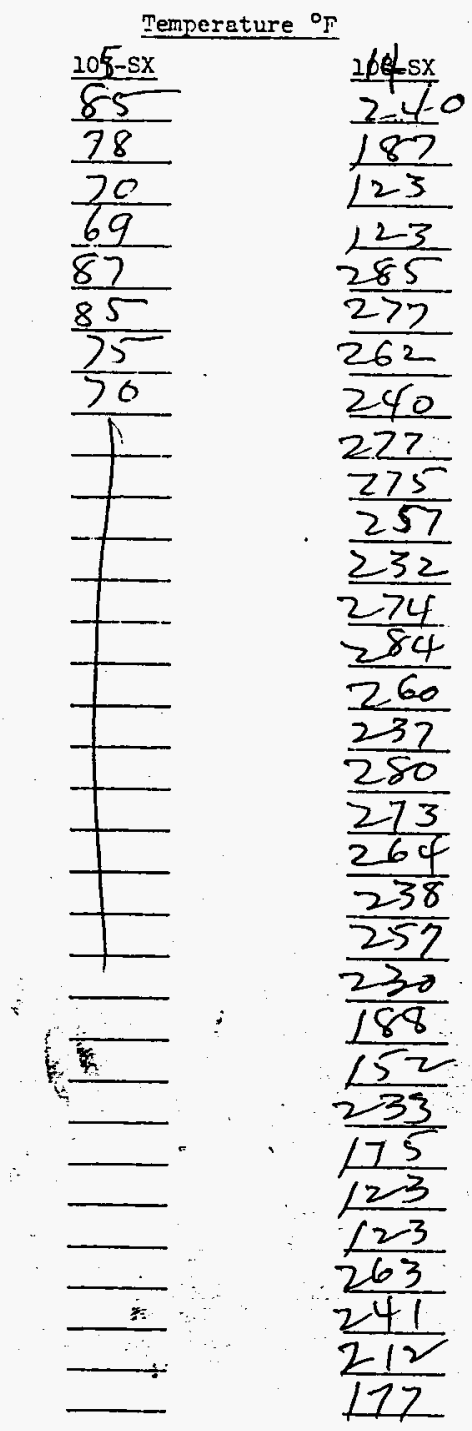




\section{1-SX SLUDGE PROFILE TEMPERATURES}

\section{TDES 10.45 DATE $2-9-12$ BEST AYALABLE COPY}

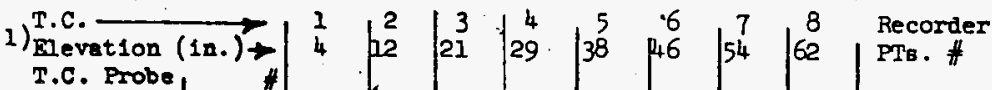

Probe Jocet:sas TK-107

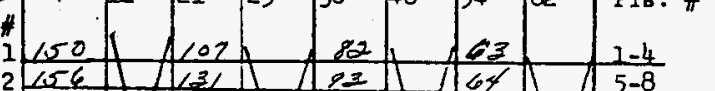

$\mathrm{TK}-1 \infty 8$

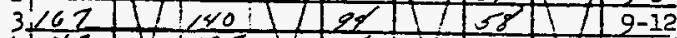

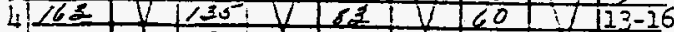

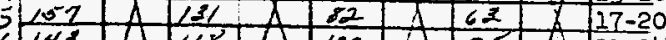

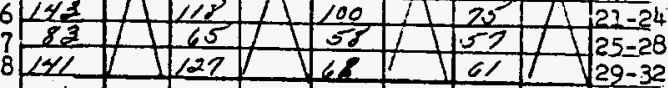

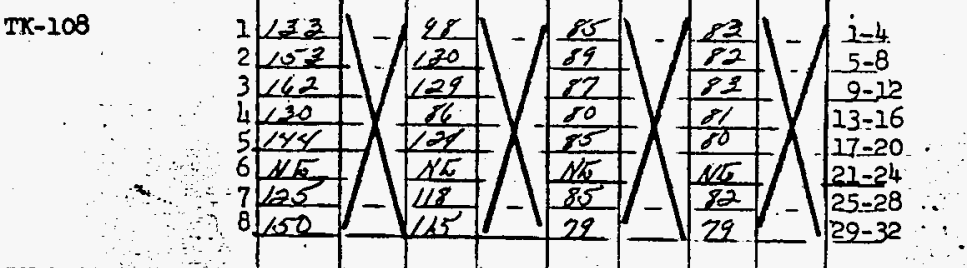

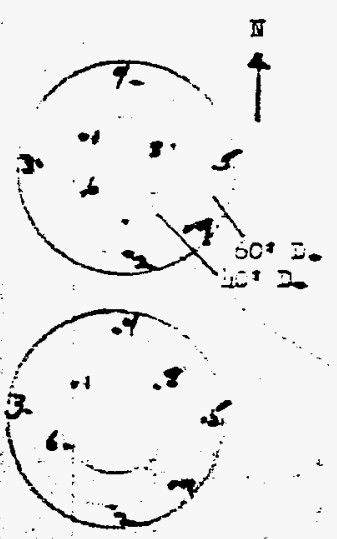

T2K-109

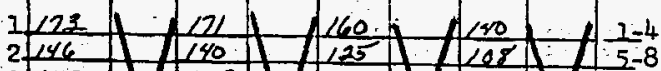

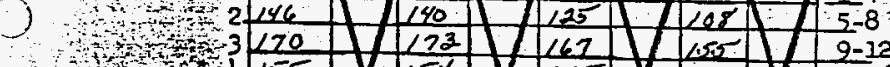

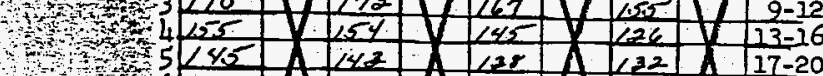

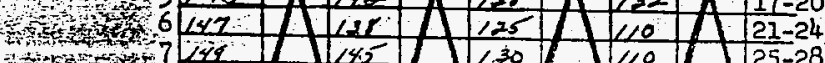

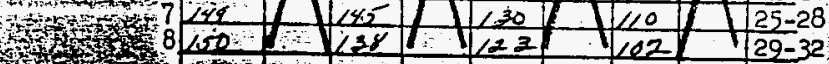
TK-110

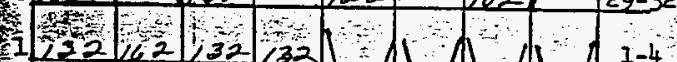

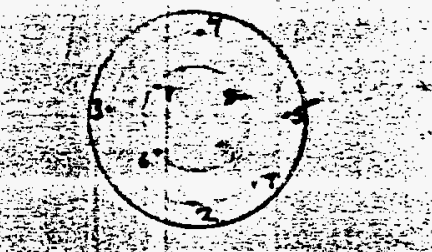

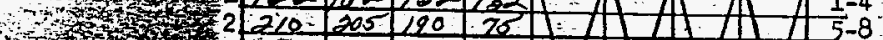

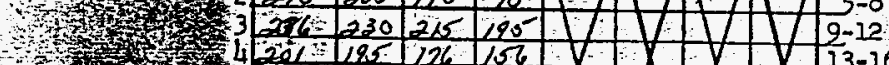

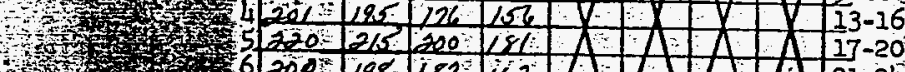

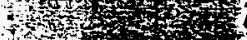

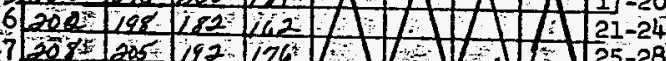

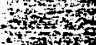

Aftitis

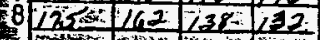
TK-II T 5 I

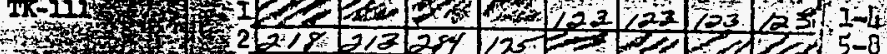

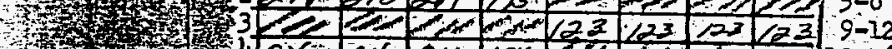

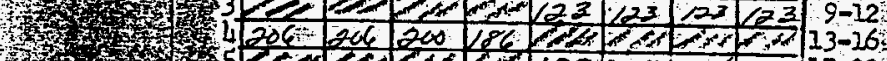

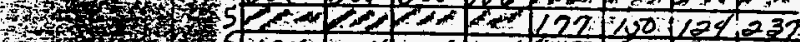

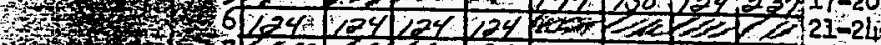

3
3

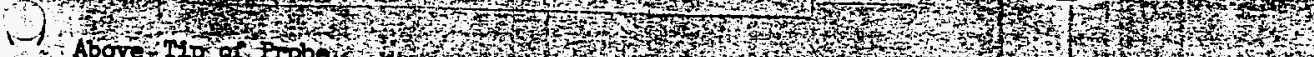

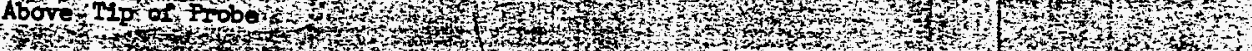
,

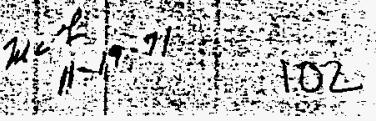


241-SX SLUDGE PROFIIE TDIPERATURES

(S.0.P. 200.3.6)

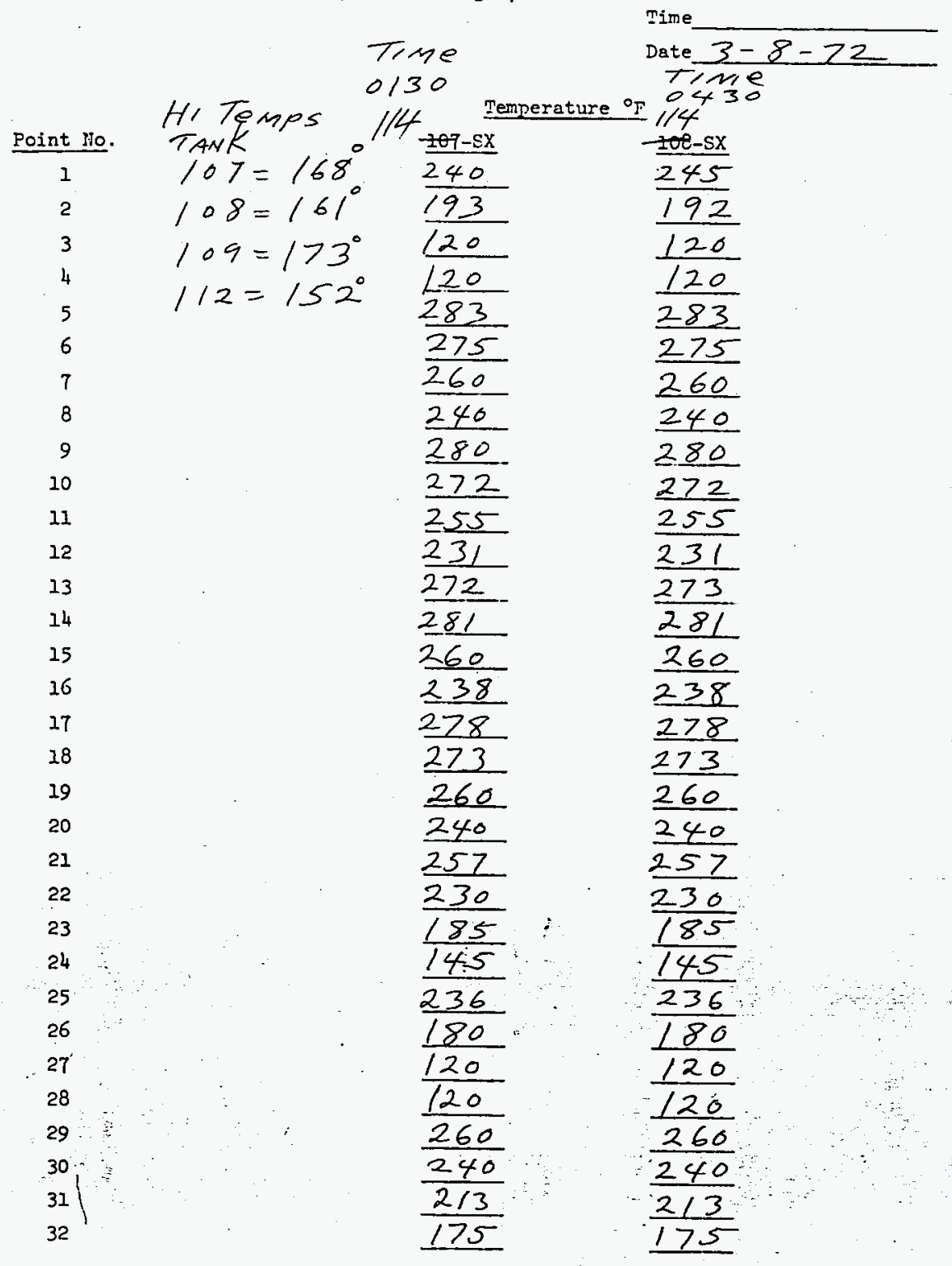




Time $\frac{1 / 5^{6}}{3-8-72}$

Point Jo.$$
1
$$$$
2
$$$$
3
$$$$
4
$$$$
5
$$

6

7

8

9

10

11

12

13

14

15

16

17

18

19

20

21

22

23

24

25

26

27

28

29

30

31

32
Temperature ${ }^{\circ} \mathrm{F}, 14$

$5 x$

245

192

121

120

2.85

276

262

240

2.80

273

256

232

274

283

262

$\frac{262}{240}$

279

275

262

239

263

230

186

145

256

177

120

119

262

$\frac{241}{213}$

176 
Point lio.

1

2

3

4

5

6

7

8

9

10

11

12

13

14

15

16

17

18

19

20

21

22

23

24

25

26

27

28

29

30

31
(1)

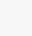

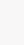

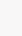

6

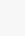

8

1

5

6

8.

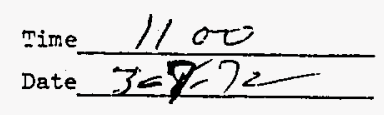




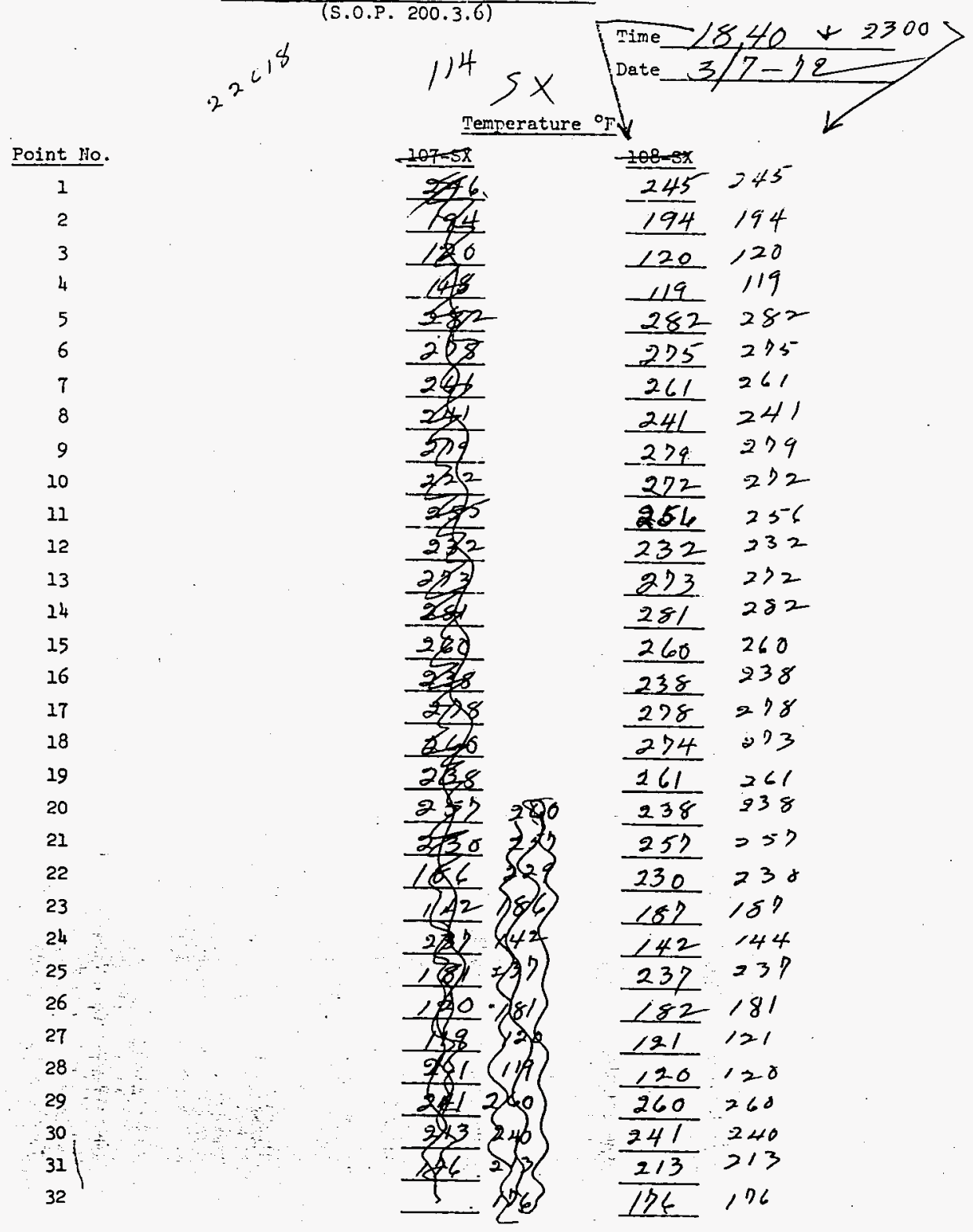


Fime

Date $3-7-72$

Point Ho.

$$
1
$$$$
2
$$$$
3
$$$$
4
$$$$
5
$$$$
6
$$$$
7
$$$$
8
$$$$
9
$$$$
10
$$$$
11
$$$$
12
$$$$
13
$$$$
14
$$$$
15
$$

16

17

18

19

20

21

22

23

24

25

26

27

28

29

30

31

32

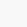

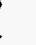

8

9

1

2

3

4

15

7

9

1

2

6

8

H, Tenips

$$
\begin{aligned}
& 107=168^{\circ} \\
& 108=162^{\circ} \\
& 109=174^{\circ} \\
& 112=154^{\circ}
\end{aligned}
$$

4

$1 / 4$ Temperature ${ }^{\circ} \mathrm{F}$ 107-sx@0130 248 200 $\frac{200}{120}$ 282

274

260

240

280

271

256

232

272

280

260

239

279

273

260

240

257

230

$\frac{250}{185}$

130

238

185

120

118

260

$\frac{260}{240}$

213

175
11400455

246

198

120

118

282

274

260

240

280

270

255

232

272

280

260

239

279

273

260

240

256

230

185

136

238

185

120

118

260

240

$2 / 3$

175 
(5.0.P. 200.3.6)

Point No.

1

2

3

4

16

17

18

19

20

21

22

23

24

25

26

27

28

29

30

31

32

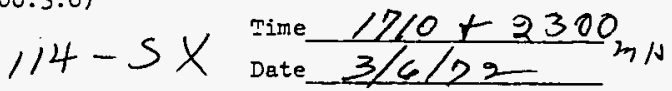

\section{Temperature ${ }^{\circ} \mathrm{F}$}

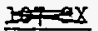

948

221

118

$-.118$

281

224

261

241

237

$\frac{211}{-256}$

232

212

279

259

238

$27 ?$

212

258

238

257

231

183

133

238

$18 \%$.

119
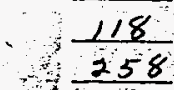

239

2

126
$100-5 x$

244

200

118

118

282

224

261

241

278

221

256

232

$2) 2$

280

260

238

218

212

259

239

$-257$

231

181

135

238

186

119
259

239

$2 / 3$

126 


\section{1-SX SLUDGE PROFIIE TMNTERATURES}

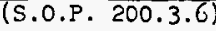

Point Jo.

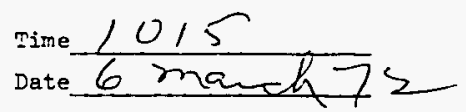

1

2

3

4

5

6

7

8

9

10

11

12

13

24

15

16

17

18

19

20

21

22

23.

24

25

26

27

28

29

30

31

32
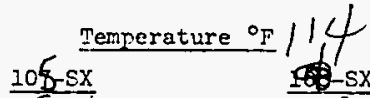

$\frac{\frac{10689}{89}}{72}$

71

71

83

76

71
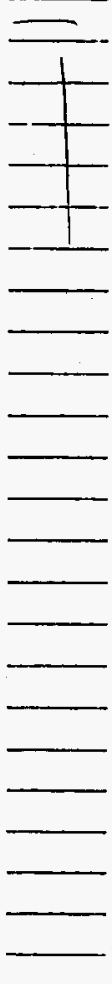

IE-Sx

$\geq 48$

202

117

116

283

$\frac{274}{262}$

$\frac{262}{242}$

274

271

$\frac{276}{178}$

178

273

281

260

$2 \longdiv { 3 9 }$

227

273

$\frac{261}{240}$

258

232

188

130

240

188

117

260

240

$\frac{214}{175}$ 


Dime $3-6-72$

Point Ho.

21

12

13

14

15

16

17

18

19

20

21

22

23

24

25

26

27

28

29

30

31

32
H, Tewips

$107=167^{\circ}$

$108=160^{\circ}$

$109=172^{\circ}$

$112=153^{\circ}$
$1 / 4$ Temperature ${ }^{\circ} \mathrm{F}$

1140200

207-sx $@ 0200$ 100-sxe

$\frac{248}{206}$

$\frac{206}{116}$

281

274

260

241

277

270

255

232

271

280

260

239

277

270

260

240

256

232

192

125

240

$\frac{240}{192}$

$\frac{117}{116}$

260

$\frac{\frac{260}{240}}{127}$ 
241-SX SLUDGE PROFILE TEMPERATURES

(S.0.P. 200.3.6)

Point No.

$$
1
$$$$
2
$$

3

4

5

6

7

8

9

20

11

12

13

14

15

16

17

18

19

20

21

22

23

24

25

26

27

28

29

$-30$

31

32
Time $\frac{1700+2300}{3 / 5 / 72}$ Temperature ${ }^{\circ} \mathrm{F}$

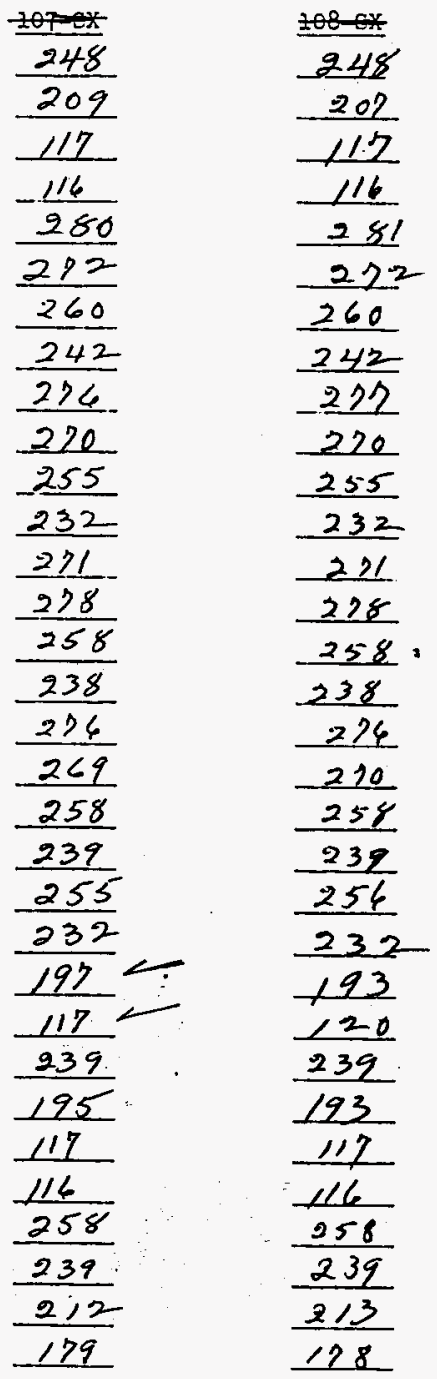


24I-SX SLUDCE PROFILE TRMIPERATURES

Time $3-5-72$

Point No.

\section{I}

2

24

25.

26

27

28

29

30.

31

32 $\frac{\text { 24-SX. SLUDCL PR.OFILE }}{(\text { S.0.P. } 200.3 .6)}$
Trme 0100 $1 / 4$ Temperature ${ }^{\circ}$
$\frac{107-5 x}{748}$
$\frac{2438}{613}$

$$
16042
$$$$
\frac{213}{118}
$$$$
115
$$$$
280
$$$$
270
$$$$
260
$$$$
242
$$$$
275
$$$$
270
$$$$
255
$$$$
232
$$$$
270
$$$$
2>7
$$$$
260
$$$$
240
$$$$
276
$$$$
270
$$$$
260
$$$$
240
$$$$
255
$$$$
234
$$$$
209
$$$$
176
$$$$
240
$$$$
200
$$$$
154
$$$$
35
$$$$
25
$$

$\frac{200}{120} 117$

$115 \quad 115$

258

240

$2 / 2$

288
114

248

$\frac{211}{117}-43^{\circ} \sin 2 d A Y S$

$\frac{115}{280}$

280

271

260

242

275

270

255

232

270

278

260

238

275

270

260

240

255

232

202

$\frac{170}{240}$

$\frac{240}{198}$

115

115

258

240

$2 / 2$ 


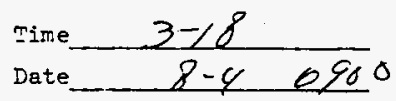

Point No.

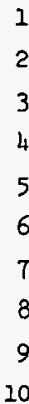

11

12

13

14

15

16

17

18

19

20

21

22

23

24

25

26

27

28

29

30

31

32
Temperature ${ }^{\circ} \mathrm{F}$

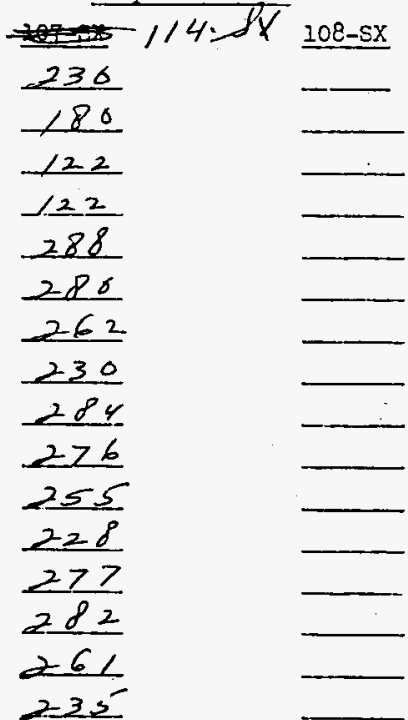

282

272

263

231

250

170

122

122

224

170

122

121

252

131

$\frac{122}{122}$ 


\section{(S.0.P.}

Point No.

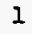

2

3

4

5

6

7

8

10

11

12

13

14

15

16

17

18

19

20

21

22

23

24

25

26

27

28

29

30

31

32

$$
14-5+
$$

$107 \mathrm{sK}$

231

181

120

$-120$

28?

299

262

236

283

$\frac{275}{255}$

$-\frac{255}{229}$

$\frac{229}{277}$

$-286$

26.1

236

282

231

$\frac{268}{237}$

257

$\frac{252}{1922}$

$\frac{192}{120}$

\begin{tabular}{l}
$\frac{120}{120}$ \\
$\frac{226}{171}$ \\
$\frac{120}{120}$ \\
$\frac{268}{191}$ \\
$\frac{120}{119}$ \\
\hline
\end{tabular}
208-E*

$3 / 18 / 32$ 
241-SX SLUDCE FROFILE TEIPERATURES

(S.0.P. 200.3.6)

Time 1220
Date $3-17-72$

Point No.

1

2

3

4

5

6

7

8

9

10

11

12

13

14

15

16

17

18

19

20

21

22

23

24

25

26

27

28

29

30

31

32
$1 / 4$ memperature ${ }^{\circ}$

108-5x

230

180

719

287

280

260

235

284

275

255

230

276

285

260

235

282

276

262

237

258

200

120

120

$\frac{120}{225}$

170.

120

120

265

$212 \mathrm{~V}$

$\frac{120}{120}$
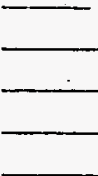

$\longrightarrow$

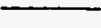

:
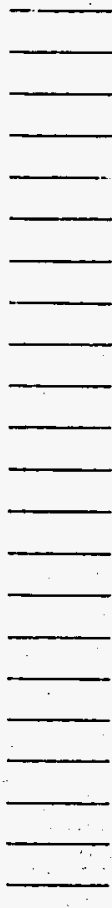

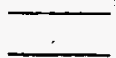


(S.0.P. 200.3.6)

Point No.

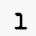

2

3

4

5

6

7

8

9

10

11

12

13

24

15

16

17

18

19

20

21

22

23

24

25

26

27

28

29

30

32.

32
0900

$7 / 4 /$ Esperature op

207-xix

232

185

$\angle 25$

124

206

228

262

232

282

225

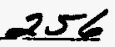

230

$\frac{250}{346}$

283

261

236

280

275

264

238

$\begin{array}{r}258 \\ 232 \\ \hline\end{array}$

197

167

226

171

24

124

264

242

$\frac{213}{184}$
Time

Date $\frac{3-<6-72}{1330}$

$1 / 45$

108-SX

232

184

121

121

282

279

262

237

282

$\frac{282}{275} \frac{256}{26}$ ydegres -

229

277

$285^{-}$

261

235

281

275

263

237

258

232

198

167

227

172

121

121

264"

242

212

184 
Point Ho.

1

2

20

21

22

23

24

25

26

27

28

29

30

31

32.

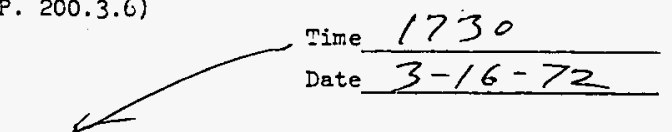

$1 / 4$ Temperature ${ }^{\circ}$ - $\mathrm{Sx}$

231

183

120

120

286

280

262

236

283

275

255

230

276

285

261

235

281

275

265

237

258

232

195

125

225

170

120

120

265

242

$2 / 3$

184 106-5x @2100

$\frac{231}{183}$

183

120

120

286

280

262

237

283

275

256

230

276

285

262

235

281

276

265

237

260 .

231

135

120

225

172

120

120

$265 v$

$\frac{242}{214}$

184 


\section{TIE 0945 DATE $3-\angle 6-72$ BEST AUASAR RDP}

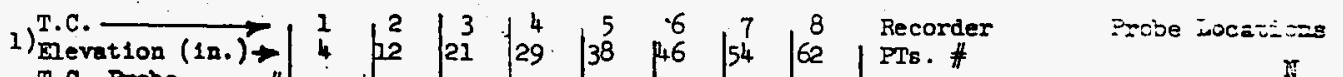

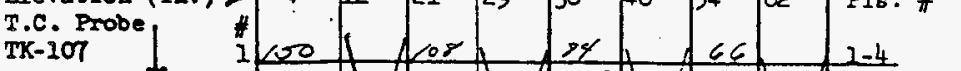

$\pi K-108$

\begin{tabular}{|c|c|c|c|}
\hline$2 \longdiv { 1 2 6 }$ & 152 & 95 & 67 \\
\hline
\end{tabular}

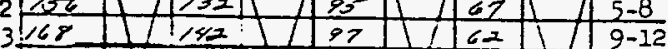

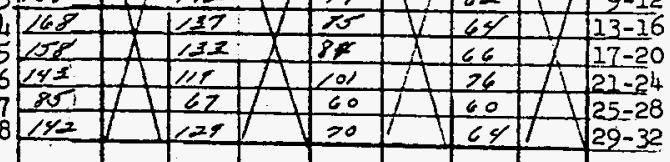
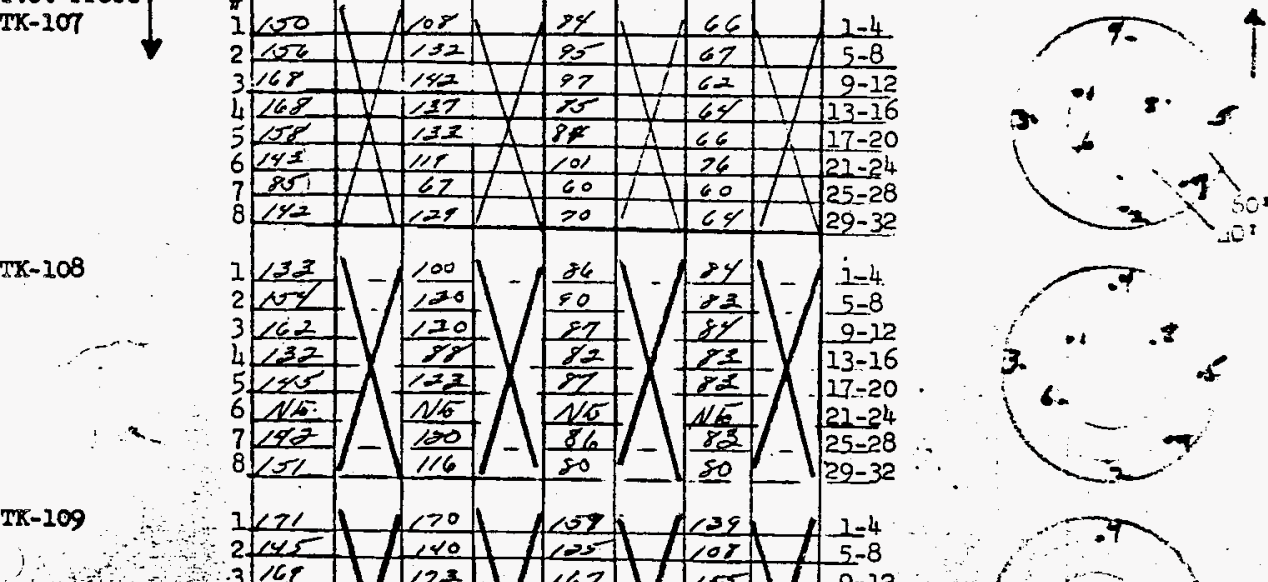

$\frac{133}{104} \mid-\left(\frac{100}{120}\right)-\left(\frac{86}{10}\right)-\left(\frac{84}{83}\right)-\left(\frac{i-4}{5-8}\right.$
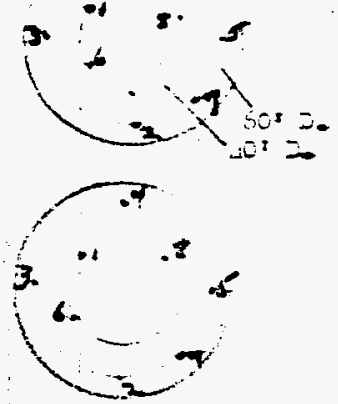

TK-109

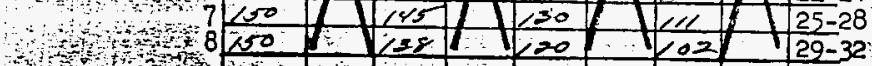

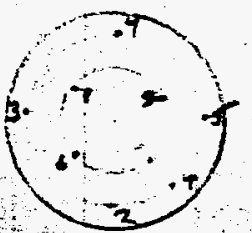

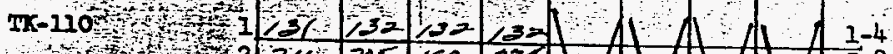

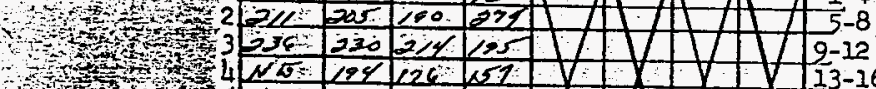

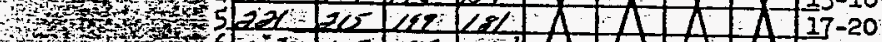

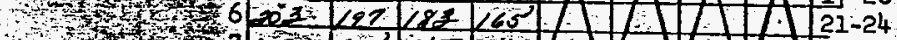

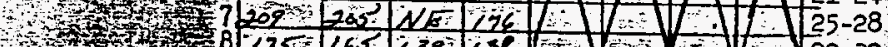

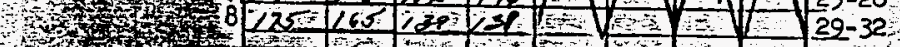
$\mathbf{T x}-211$

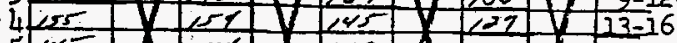

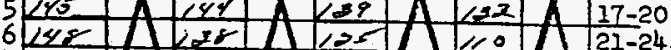

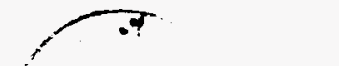

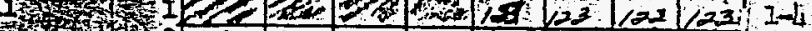

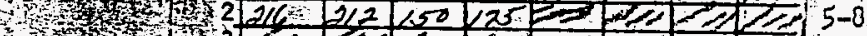

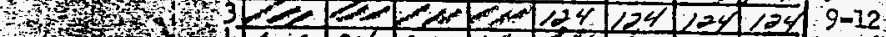

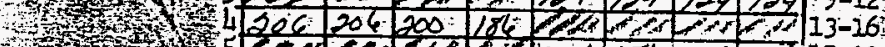
3
3

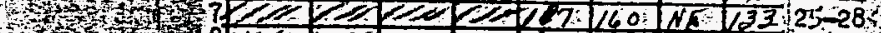

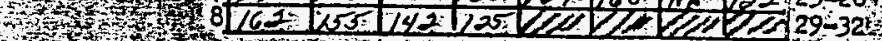
$1+1$
$6+3$
4
4

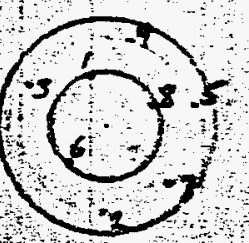

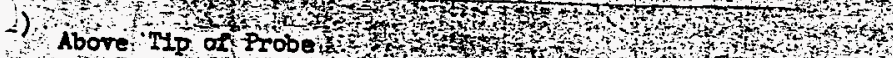

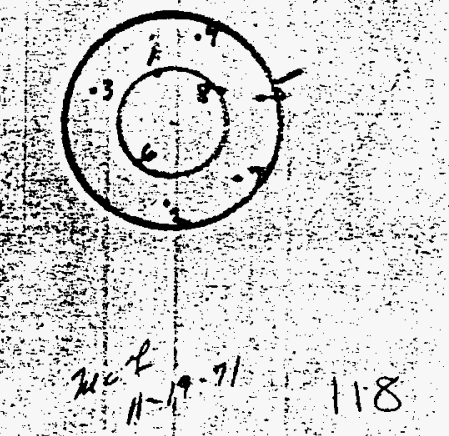


WHC-SD-WM-D̈P-ż32, Rev. 0

\section{BEST AMALABLE COPY,$-10-72$ \\ $-2-$}

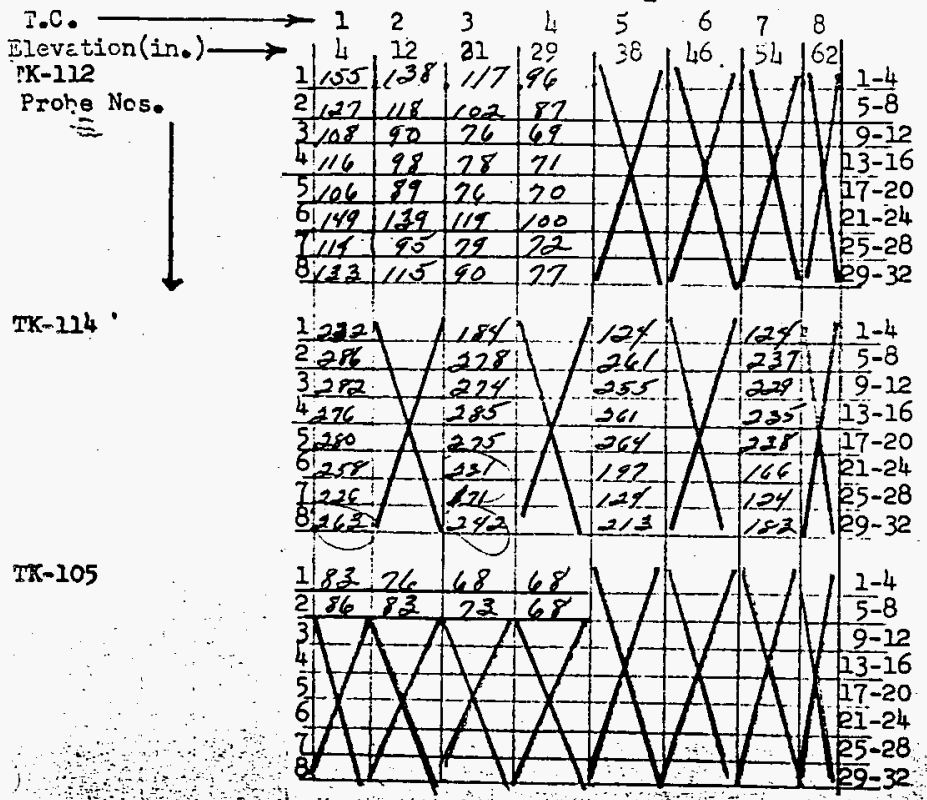

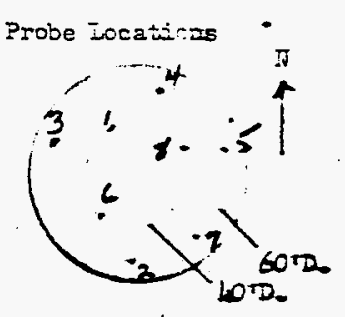
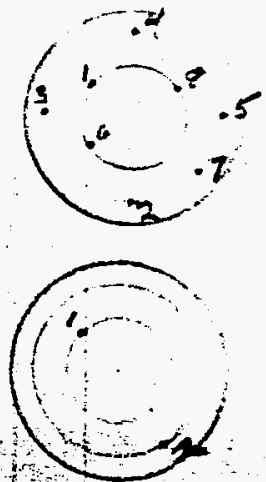

10

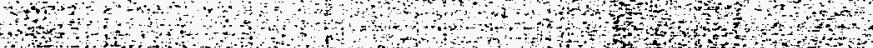

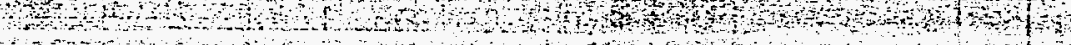

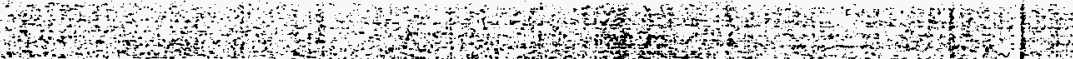

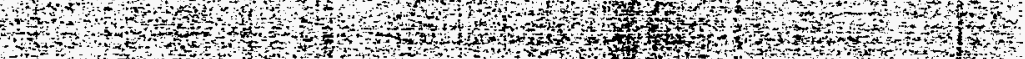

$n$
$n$

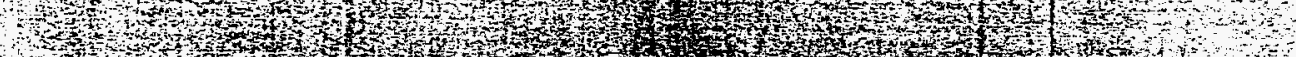

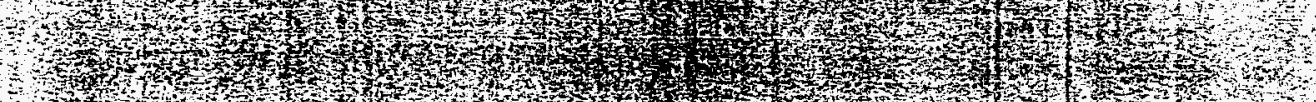

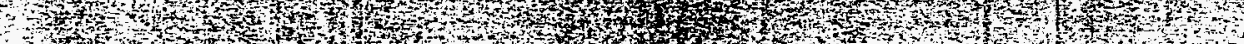

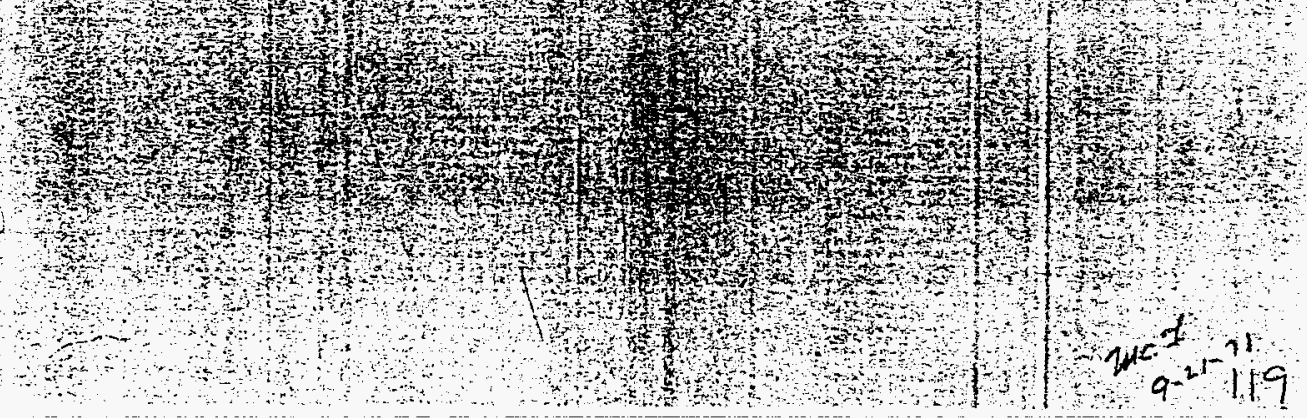


(S.0.P. 200.3.6)

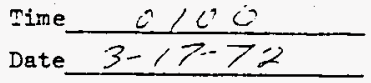

Point No.

1

2

3

4

5

6

7

8

9

10

11

12

13

14

15

16

17

18

19

20

21

22

23

24

25

$26^{\circ}$

27

28

29

30

31

32
$1 / 4 \frac{\text { Temperature }{ }^{\circ} \mathrm{F}}{\mathrm{x}}$ $107-x$

231

182

119

$-11+$

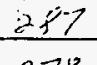

$\frac{278}{262}$

$\frac{212}{607}$

$\frac{237}{253}$

255

239

276

285

262

236

282

276

269

237

258

227

123

120

226

172

120

$\frac{119}{264}$

264

243

$2 / 3$

184
$206-3 x$

7.31

182

$120+1$

$119+1$

287

278

262

237

283

275

$256+1$

279

$277+1$

285

762

236

282

376

$263-6$

$238+1$

$759+1$

$217-10$

$120-3$

$119-1$

236

172

120

119

264

$238-5$

$125=-88$

$120-64$ 


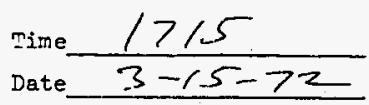

Point No.

1

2

3

4

5

6

7

8

9

10

11

12

13

14

15

16

17

18

19

20

21

22

23

24.

25

26

27

28

29

30

31

32
$1 / 4$ Temperature ${ }^{\circ} \mathrm{T}$

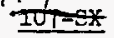

108-5X

231

185

124

124

285

278

242

237

282

275

255

$2 \frac{25}{30}$

275

285

260

235

280

275

265

238

258

$230 \mathrm{~V}$

$\frac{230}{195}:$

$\frac{195}{165}$

226

$\frac{272}{172}$

124

124

$265 \mathrm{~V}$

$242 v$

212

188 


$$
\text { (S.0.P. 200.3.6) }
$$

Point No.

1

2

3

4

5

6

7

8

9

20

11

12

13

24

15

16

17

18

19

20

21

22

23

24

25

26

27

28

29.

30

31

32

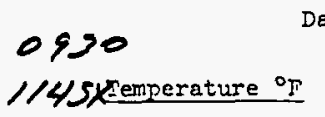

207 S4

232

$\angle 86$

127

126

286

277

262

237

281

274

$25 \%$

230

276

28

261

256

280

375

264

239

258

231

196

165

128

273 .

126

126

264

242

$2 / 3$

183

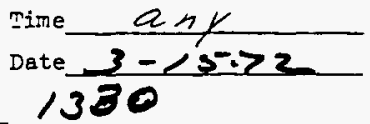

$1 / 5,5$

232

185

125

125

226

228

262

237

282

274

256

230

276

284

261

235

280

274

264

238

257

231

196

164

227

173

124

124

264

242

213

182 


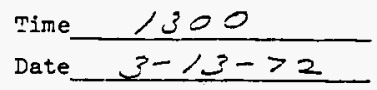

Point No.

1

2

3

4

5

6

7

8

9

10

11

12

13

14

15

16

17

18

19

20

21

22

23

24.

25

26

27

28

29

30

31.

32
$1 / 4$ Temperature of

\%

108-5x

$\frac{\frac{235}{185}}{\frac{125}{125}}$

285

278

262

239

283

275

256

230

276

284

260

236

280

274

264

238

258

230

$\frac{193}{160}$

229

173

125

125

$-264$

241

$\frac{212}{181}$
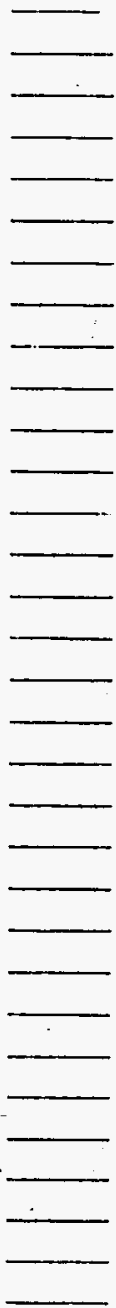
241-SX SLUDGE PROFILE TDMPERATURES

(5.0.P. 200.3.6)

Point No.

1

2

3

4

5

6

7

8

9

10

11

12

13

14

15

16

17

18

19

20

21

22

23

24

25

26

27

28

29

30

31

32

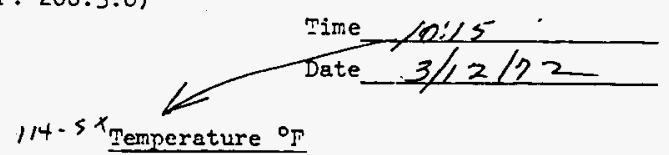

$107-5 x$

$\frac{237}{186}$

125

125

255

211

$2<1$

$\frac{238}{282}$

$\frac{282}{214}$

256

230

275

$+2523$

260

237

218

213

263

238

$2 \geq 257$

228

$192:$

158

230

135.

125

125

$\frac{262}{242}$

242

$\frac{213}{186}$ 
241-SX SLUDGE PROFILE TMUPERATURES (5.0.P. 200.3.6)

Point No.

1
2
3
4
5
6
7
8
9
10
11
12
13
14
15
16
17
18
19
20
21
22
$126 \quad 22$

$-100$

15

$15=27$

- Ifo

iff

$j \operatorname{los}$

i i
28.

29

30

31

32

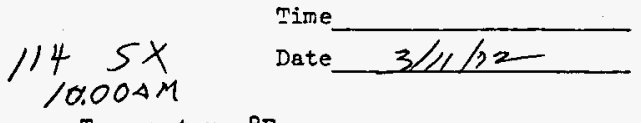

Temperature ${ }^{\circ} \mathrm{F}$

$\frac{107-5 X}{3}$

108-SX

$\frac{238}{186}$

124

$-\frac{124}{284}$

2)?

261

282

$\frac{204}{255}$

230

$\frac{215}{284}$

259

237

218

293

$\frac{262}{238}$

257

228

190

155

$\frac{232}{105}$

$\frac{135}{124}$

124

262

241

212

179
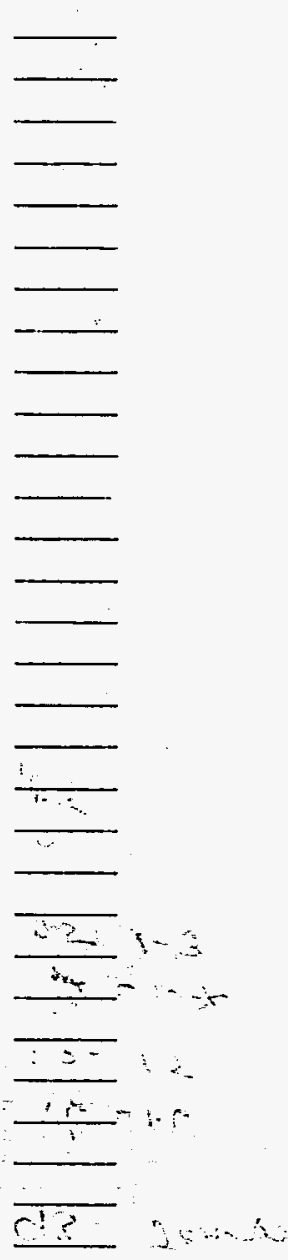

$\theta-7 y$

$2-192$

$\alpha-18$

$3=115$

$=120$

$+$

$1-121$ 


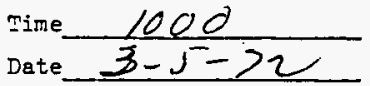

Point lio.

$$
1
$$$$
2
$$

3

4

5

6

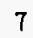

8

9

10

11

12

13

14

15

16

17

18

19

20

21

22

23

24

25

26

27

28

29

30

31

32

33
$1 / 4$ Temperature ${ }^{\circ} \mathrm{F}$

30t-8x

248

211

112

$-115$

280

223

260

242

$2>6$

$2>0$

355

232

$5>0$

$1>8$

259

238

276

269

259

$\frac{248}{258}$

255

232

200

$\frac{200}{117}$

240

197

116

115

$\frac{115}{259}$

239

$2 / 3$

162

$\frac{162}{17 s}$

$108-5 x$
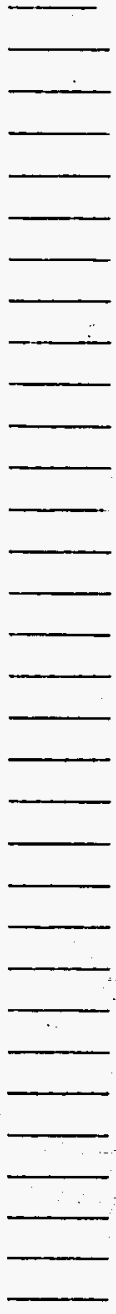
241-SX SLUDGE PROFILE TMIPERATURES

(5.0.P. 200.3.6)

Point No.

1

2

$3 \quad 36$

424

5

6

7

8

9

10

11

12

13

14

15

16

17

18

19

20

21

22

23

24

25

26

$.27 \quad 32$

$28 \quad 24$

29

30

31

32
Trane $09001 / 4$ Temperature ${ }^{\circ} \mathrm{F}$ 107-SX

$\frac{248}{215}$

215

142

$-\angle 20$

280

271

261

245

275

269

256

231

270

276

260

140

274

267

260

241

255

노

$\frac{102}{17}$

175

241

201.

131

120

257

239

$2 / 2$

164
Time

Dete $3-4-72$ $2 \widehat{100}$ 7 me $114 \times 73000$ int $\mathrm{SX}$ 248 श48 24824 $2 / 5 \quad 214 \quad 213 \quad 21$ $137 / 33 \quad 12512$ $118 \quad 118 \quad 114 \quad 114$ $280 \quad 250 \quad 280 \quad 28$ $1>1272 \quad 29227$ 261226126 $244 \quad 244 \quad 24324$ उ>s $216 \quad 216$ 20 26927027026 256 श56 256 25 $132233 \quad 23223$ 27027027027 $276298 \quad 27727$ 16026026026 3ㅇ 239239 23; 224275275216 $267 \quad 268 \quad 268 \quad 26$ : $259.260 \quad 260261$ I4l 242 .42 24: 155 256 256251 $132 \cdot 233 \quad 233 \cdot 23$ 502 202 202205 175 137 137 13. $241242 \quad 24224:$ $202202 \because 20120$. $128 \cdot 125 \cdot 123,12$ $\therefore 119 \quad 118 \quad 117 \quad 11$ 257 258 25825 $239 \cdot 238.23823$ ! $2 / 2=13221=$ $184,184,184,8$ 
241-SX SLUDGE PROFILE TDIPERATURES

(5.0.P. 200.3.6)

Point lio.

1

2

3

4

5

6

7

8

9

10

11

12

13

24

15

16

17

18

19

20

21

22

23

24

25

26

27

28

29

30

31
Tine ol:00

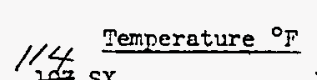

$267-5 x$

247

215

150

125

280

280

270

260

245

275

270

257

234

270

276

260

240

274

267

260

242

255

232

$\frac{202}{175}$

241

205

138

125

257

240

$2 / 2$

187
Time

Date $3-4-72$ Tine $114=0500$ $208-5 x$

247

$2 / 5$

1452

125

280

270

260

245

275

270

257

$233-$

270

276

260

240

274

267

260

242

255

232

202

175

241

205

$140=$

125

257

240

$2 / 2$

$184=$ 
Point Io.

1

2

3

4

5

6

7

8

9

10

II

12

13

14

15

16

17

18

19

20

21

22

23

24

25

26

27

28

29

30

31

32
$11^{4-5 X @ 170+2}$ temperature 25 $2300 \mathrm{~L}$

Time

Date $3 / 3 / 32$

$248 \quad 248$

$\begin{array}{lll}216 & 216 \quad \frac{216}{157}\end{array}$

$157152 \quad 15.1^{\circ}$

$135130 \quad 128$

278 278 278

$271230 \quad 270$

$\frac{262}{248}=262 \quad \frac{262}{146} \mathrm{~V}$

$276-275 \quad 275$

$269 \quad 268 \quad 268$

$258 \quad 258 \quad 257$

$233 \quad 233 \quad 233$

$269 \quad 269 \quad 269$

$277=33 \quad 231$

$\frac{261}{242} 242 \frac{261}{242}$

$273 \quad 273 \quad 213$

$267=67 \quad 267$

$260260 \quad 260$

$243.243 \quad \frac{240}{242} \mathrm{~V}$

256 256 256

$232232 \quad 232$

202262 202

$125-195 \times 175$

$242 \quad 242,242$

205.2265 205

148 143 141

$135130 \quad 129$

258 258 $\frac{128}{258}$

238 238 239

$2 / 2$ 2,2 $\frac{212}{212}$

$183 / 83 \quad \frac{183}{183}$ 
241-SX SLUDGE PROFILE TTEPERATURES

Point No.

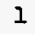

2

3

4

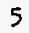

6

7

8

9

10

11

12

13

14

15

16

27

18

19

20

$22^{2}$

$22+7$

23

24

25

26

27
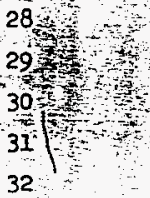

(S.0.P. 200.3.6)

$$
14 S \lambda \text { Date } 3-3-72
$$

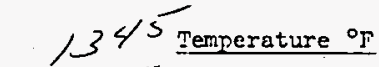

- 500

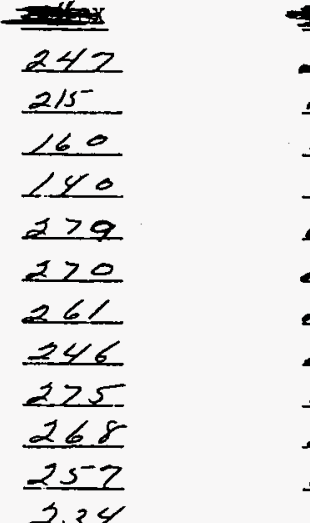

234

262

276

260

242

$2 \geq 3$

266

$\$ 60$

243

256

232

$\frac{202}{174}$

243

$205^{-}$

$1 s-4$

140

258

$\frac{239}{212}$
舟

248 216 $13-3$ 136 280 27 262 247 275 269 252 234 269 222 261 242 224 267 260 $-213$ 256 $2 ? 2$ 201 124 243 204 151 137 $2 s^{-2}$ 232 212 183 
241-SX SLUDGE PROFILE TRMPERATURES

(S.0.P. 200.3.6)

Point No.

1

2

3

4

5

6

7

8

9

10

11

12

13

14

15

16

27

18

19

20

21

22

23

24

25

26

27

28

29

30

31

32
$1 / 4$ - $\begin{aligned} & \text { Temperature } \\ & \text { op }\end{aligned}$

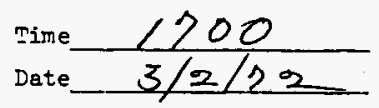

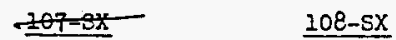

248

212

162

142

278

$2<3$

262

248

215

268

$-258$

234

268

216

$2<2$

243

2) 3

267

260

242

255

232

201

123

.243

205.

152

142

258

$\frac{239}{282}$

183 


\section{TAMB 1330 DAFB $3-2 \geq 2$ BEST AVALILBLE COPY}

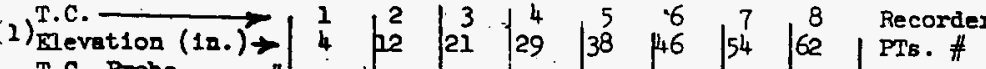
T.C. Probe $T K=107$

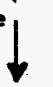

$\mathrm{TK}-108$

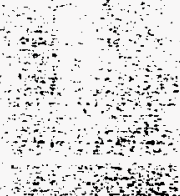

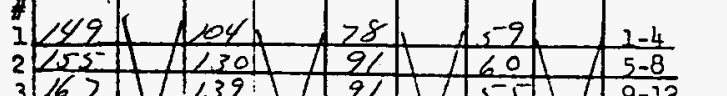

(1)

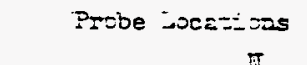

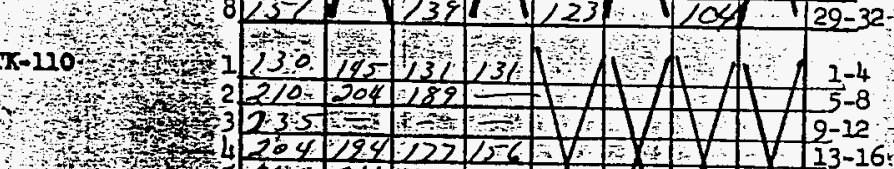

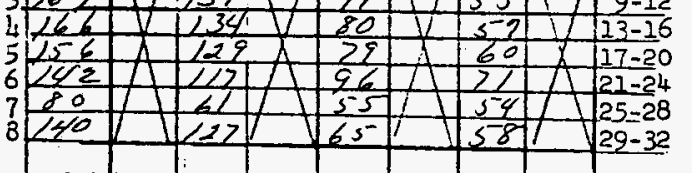
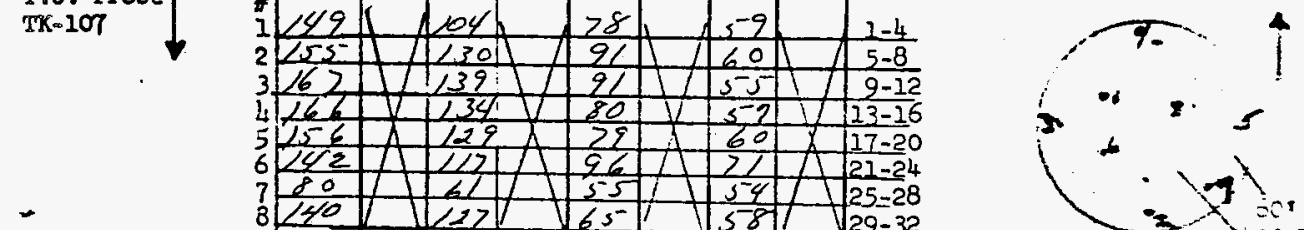

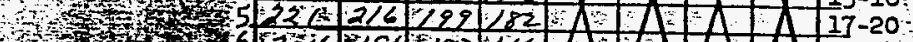

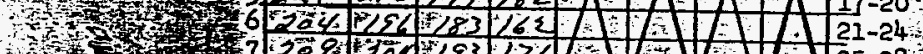

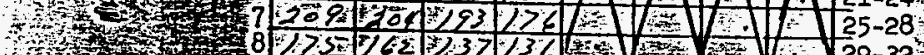

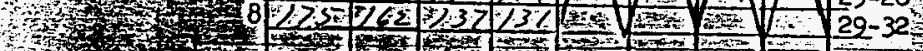

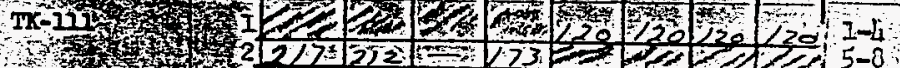

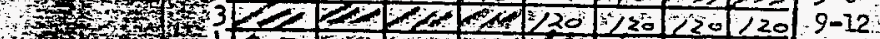

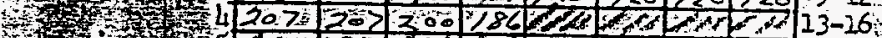

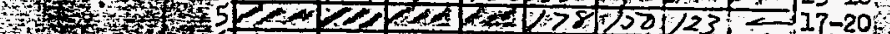

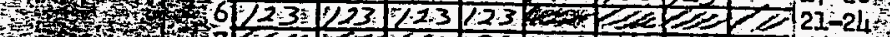

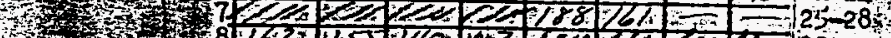

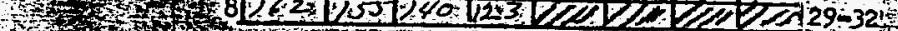

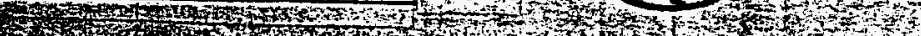

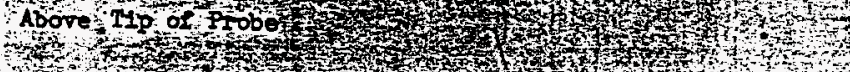

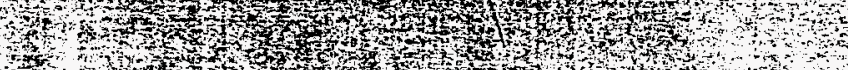

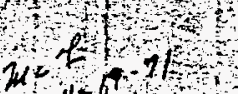

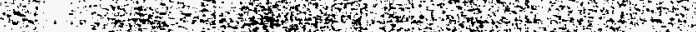

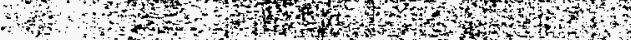



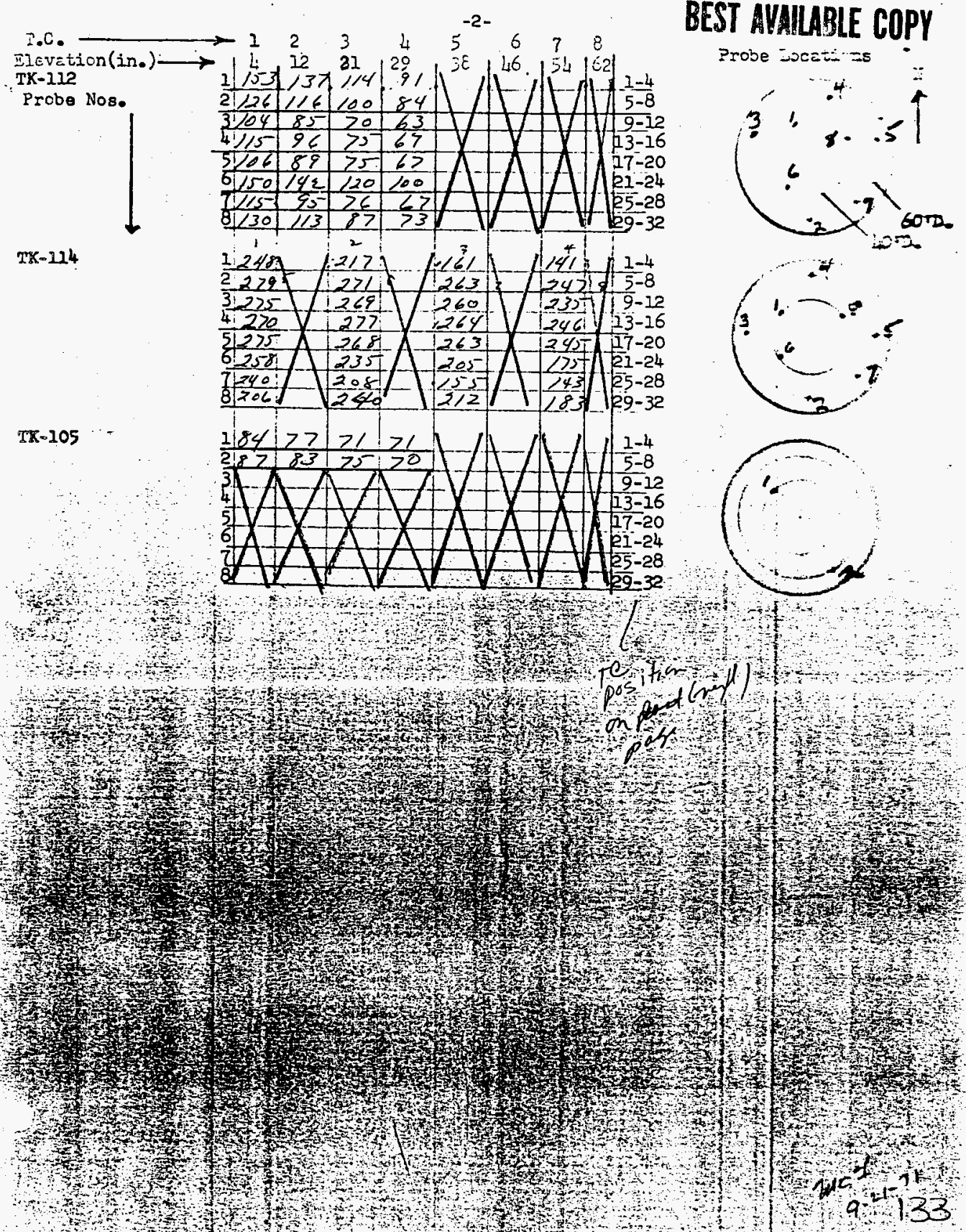
241-SX SLUDGE PROFILE TDMPERATURES $(5.0 . P \cdot 200.3 .6)$

Point No.

1

2

3

4

5

6

7

8

9

10

11

12

13

14

15

16

17

18

19

20

21

22

23 24 25 26

27 28

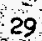

30

31

32

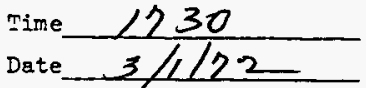

$5 x$

$1 / 4$ Temperature ${ }^{\circ}$

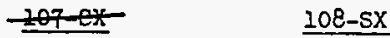

$248 \quad 1$

3,42

139 "

1424

27?

268

262

249

236

269

258

234

268

216

262

244

$2 ? 3$

266

259

242

255

231

206

122
243

207

$\frac{152}{142}$

258

$\frac{238}{212}$ 